University of Zurich

Department of Economics

Working Paper Series

ISSN 1664-7041 (print)

ISSN 1664-705X (online)

Working Paper No. 375

\title{
Inequality in Models with a Competition for Market Shares
}

Andreas Hefti and Julian Teichgräber

January 2021 


\title{
Inequality in Models with a Competition for Market Shares
}

\author{
Andreas Hefti and Julian Teichgräber*
}

January 4, 2021

\begin{abstract}
This paper develops a framework to systematically study how changes in market conditions affect the equilibrium inequality between heterogeneous agents. By stating our setting as a "competition for market shares", we can derive inequality predictions for vastly different competition models. This approach allows us to identify a common structure, e.g., in monopolistic competition, perfect competition, or competition for prizes, that explains why these models deliver similar inequality predictions. We apply our results to problems from trade, competition theory, consumption inequality, political economics and marketing, and relate some of the predicted inequality patterns to empirical evidence.
\end{abstract}

JEL Classification: C65, D30, D41, E10, L11, M37

Keywords: Inequality Analysis; Market Shares; Power Functions; Monopolistic Competition; Perfect Competition; Competition for Prizes

*Corresponding Author: Andreas Hefti, Bahnhofplatz 12, CH-8400 Winterthur, heft@zhaw.ch. Author affiliations: Hefti: School of Management and Law (ZHAW) and Department of Economics (UZH). Teichgräber: Department of Economics (UZH). We wish to thank Armin Schmutzler, Peter Neary, David Wettstein, Kfir Eliaz, Reto Foellmi, Shuo Liu and Ariel Levy, and the participants of seminars and conferences sessions for many valuable comments. 


\section{Introduction}

It is a common empirical observation that the dispersions of market shares, payoffs, revenues, consumption or income display a substantial amount of inequality. One source of such marketborne inequality are differences between the various agents, e.g., in endowments, abilities, information, inclinations or alike.

What seems to be less understood is the extent to which the market inequality originating from ex ante heterogeneous agents is influenced by market conditions that affect one or all agents. To illustrate the latter in case of firms, this could amount to the total disposable consumer income, the overall level of productive efficiency, a sales tax, the level of available production resources, the overall propensity of consumers to substitute between products, or the advertising affinity of consumers. A change in such market conditions influences each firm, and the corresponding responses depend on the direct impact of the changed condition alongside with the reactions of all other market participants. This amalgam of effects makes it hard to analyze how inequality in market shares or payoffs evolves.

In this article, we study how the dispersion of market shares and related measures depends on exogenous market conditions. In the general setting, an arbitrary number of ex ante heterogeneous agents choose their actions such as to maximize their payoffs. The dispersion of market shares then is the equilibrium consequence of these actions. We express such a competitive situation as a "competition for market shares", where the agents compete directly in terms of market shares. This setup can accommodate different paradigms of competition, such as monopolistic competition, price competition or competition for prizes, with a contextual interpretation of what market shares are. Formulating these models as a competition for market shares allows us to identify structural relations between the otherwise different models of competition, which provides valuable guidelines for understanding conceptual similarities - or disparities - in the respective equilibrium inequality effects.

A change in a market condition may exacerbate or reduce the existing market inequality, and one might care about such effects for several reasons. For instance, a regulating authority may want to assess which market-level interventions (e.g., taxes or quotas) affect market concentration or the sales distribution on the supply-side, or whether it moderates consumption inequality on the demand-side. Policy makers frequently need to render an account for the inequality in the chances of winning a grant, patent or a monopoly franchise. Sports tournament designers often care about finding a reward scheme that makes the competition most unpredictable. For empirical work it may be helpful to know whether a certain inequality pattern in a market is "robust" in thus that specific distributional assumption about the ex-ante agent heterogeneity is not decisive. Such insights could be particularly useful if the data does not allow to identify the ex-ante agent heterogeneity, such as producer-side cost functions.

We derive a set of formal results to analyze the equilibrium inequality effects if a market condition changes, where this market condition can affect the payoffs of all or only some agents - we only require that there is no equilibrium "leap-frogging" by some agents in terms of their mar- 
ket shares. We say that a market condition induces inequality effects whenever the equilibrium market shares are not invariant to variations of this condition.

Our first results characterize whether any such inequality effects arise for a given market condition $x$. We prove that this question can be completely answered by restricting attention to a local property - the "direct-aggregative effect" - of an equilibrium equation, which originates from the agent-wise optimality conditions and is "type-recursive" (i.e., recursive in the agents' identities). A change in $x$ can affect marginal costs and benefits directly as well as indirectly via the aggregate response of all agents - the direct-aggregative effect is the net effect of the two.

In our framework, the ex ante agent heterogeneity is necessary and sufficient for a non-uniform dispersion of market shares to arise - but does this dispersion become more or less equal if a certain market condition changes? The second set of results addresses this question by centering on equilibrium rotations of the market share dispersions. These rotations can be directed "outward" or "inward", the former implying an increasing and the latter a decreasing equilibrium inequality.

We show that the direct-aggregative effect can determine the existence of rotations, and their directions. This yields a powerful tool for studying inequality effects, as the direct-aggregative effect is a local property of a single equation which nevertheless is informative about a global property of a distribution.

For example, we use this insight to prove that the elasticities of marginal costs alone determine whether a rotation occurs following an increase in a "level variable". A level variable is a market condition that affects the marginal benefits (or costs) of all agents by the same proportion, thereby inducing the same incentives for all agents to aspire for a larger market share. If the agents with a larger current market share are also those featuring a less elastic marginal cost function, then an increase in a level variable causes an outward rotation of the market share function, reflecting that these agents can adapt better to the new situation by means of their costs.

The notion of a rotation is related to the concept of Lorenz dominance, which plays a central role in empirical inequality analysis. Specifically, an outward (inward) rotation of the market shares means that market inequality has increased (decreased) as measured by any Lorenzconsistent inequality measure (such as the Gini coefficient).

Our general analysis identifies a central role of power functions. If the type-recursive equation is such that the "costs" and "benefits" associated with attaining a certain market share are power functions, and the direct-aggregative effect pertaining to a market condition has the same sign for all agents, then any inequality effect must take on the form of a rotation that is monotonic in agent type. That is, the changes in market shares must be ordered such that stronger agents must gain (or lose) more, in a relative sense, compared to any weaker agent. Such insights matter as power functions arise in many economic models and similar applications Newman, 2005).

With power functions, more can be said about the relation between the dispersion of market 
and payoff shares. For example, we show that if the revenues per unit of market shares are identical across agents, amounting to a symmetric type of competition, then the market and payoff shares must always coincide. By contrast, if agents with larger market shares also earn a higher revenue per unit of market share, then the payoffs are less equally dispersed than the market shares. Further, we derive sufficient conditions for the market and payoff shares to respond similarly to changes in a market condition.

We put our inequality tools into action in several applications from different fields, such as monopolistic competition, perfect competition, and a class of models where agent interaction is described as a competition for prize $1^{1}$ While these models feature substantially different forms of competition, we observe a great deal of likeness in the inequality effects they predict. The reason for this finding is that the underlying competition for market shares follows a common formal structure, despite the different competition settings.

Our approach provides several novel insights within the specific applications we consider. As of its basic contribution, our article complements a recent literature focusing on how the distribution of the ex ante agent heterogeneity affects the dispersion of certain equilibrium quantities. For example, Mrázová et al. (2016) relate the distribution of firm sales and markups to the underlying distribution of technology in a monopolistic competition setting, or Jensen (2017) studies how exogenous changes in the ex-ante distribution, such as increased uncertainty in a prior belief, may affect certain outcomes. By contrast, we predominantly ask when a change in the economic environment makes advantaged agents more dominant holding the ex ante agent heterogeneity fixed, or when it moderates market concentration and diminishes the market advantages originating from favorable ex ante conditions of certain agents. Nevertheless, our approach is also suitable to study the inequality effects induced by a change in the ex ante agent heterogeneity itself.

Article structure We define the concept of a competition for market shares in Section 2, and introduce the key definitions, such as the ex ante agent heterogeneity (the "agent types") or the notions of a market condition or inequality effects. In Section 3 we derive the type-recursive equation which characterizes the possible inequality effects, and define the notion of a rotation as a key inequality concept for our applications. We apply our formal approach to a number of examples in Section 4. All proofs of the formal claims made in the main text can be found in Appendix A, while Appendix B contains additional results pertaining to our general analysis.

\section{Competition for Market Shares}

Let $I=[0,1]$ denote a set of agents, where $i \in I$ is a particular agent. An agent's payoff function has the form

$$
\Pi(i)=p(i) V(i)-\Phi(i)
$$

\footnotetext{
${ }^{1}$ In Appendix B.8, we use our approach to explore the inequality effects of firm-driven changes in product perception, or the distributional impact of an import tax on domestic and foreign firms market shares in international trade. Also see Hefti and Lareida (2020) for a stand-alone application in case of competitive advertising, and Hefti et al. (2020) for the case of a dynamic model.
} 
where $p(i)$ is the market share of $i, V(i)$ the value earned per unit of market share, and $\Phi(i)$ are expenditures. Each agent can choose an action $t(i) \in \mathbb{R}_{+}$to maximize her payoff. In general, this action affects each component of (1), together with the joint actions of all agents as summarized by a quantity $T \in \mathbb{R}_{+}$. Further, the payoff function of at least one agent depends on an exogenous parameter $x \in X$, where $X$ is an open interval in $\mathbb{R}$. Thus, the payoff generally is a function 2

$$
\Pi(i, t(i), T ; x)=p(i, t(i), T ; x) V(i, t(i), T ; x)-\Phi(i, t(i) ; x) .
$$

The way how $t(i)$ and $T$ enter (1) pins down the details of how the agents are engaged in a competition for market shares. In many (but not all) of our applications, $T$ is the sum of all chosen actions. That is, if the actions $t(i)$ chosen by each agent $i \in I$ are summarized by the action profile $t: I \rightarrow \mathbb{R}_{+}$, then $T=\int_{I} t(i) d i$. More generally, $T$ is determined by an aggregator function $Z(t)=T$ for a set of viable action profiles $3^{3}$ As $p(i)$ are market shares, we impose the identifying assumption that they must add up to one

$$
T=Z(t) \Longleftrightarrow \int_{I} p(i, t(i), T ; x) d i=1
$$

In words, condition (3) says that the individual market shares add up to one for a given aggregate $T$ if and only if the action profile $t$ exactly generates $T$. We call $\left(\{\Pi(i)\}_{i \in I}, Z\right)$ a competition for market shares if each $\Pi(i)$ is of form (2) and $Z(\cdot)$ verifies (3). Many economic models are competitions for market shares in this sense, as we make explicit in Section 4.

\subsection{Equilibrium Market Shares}

An equilibrium in $(\{\Pi(i)\}, Z)$ is an action profile $t$ and a quantity $T$, such that for each $i \in I$, $t(i)$ maximizes (2) given $T$, and $T$ is endogenously determined by $Z(t)=T$.

This equilibrium definition encompasses, e.g., monopolistic competition equilibria, Walrasian equilibria, or equilibria in large aggregative games (see Section 4). Further, it is consistent with equilibria in the "Global Games" literature (see, e.g., Morris and Shin, 2002). A common aspect of all these equilibrium definitions is that each agent takes an aggregate $T$ as given when optimizing her payoff, while $T$ is endogenous to the model. Such a property appears reasonable with a large number of agents Alos-Ferrer and Ania, 2005, Acemoglu and Jensen, 2010; Hefti, 2016: Camacho et al. 2018), and it simplifies the formal analysis of the inequality effects that this paper cares about. In Appendix B.5 we show that our analysis is generally not confined to this behavioral assumption: Our approach can be adjusted to encompass the case of Nash equilibria in aggregative games as well.

Competition for Market Shares An equilibrium $(t, T)$ generates a certain dispersion of market shares $p(\cdot)$. To systematically study the equilibrium inequality effects caused by a parameter $x$, we derive an equivalent characterization of equilibrium, where the agents compete

\footnotetext{
${ }^{2}$ Agent index $i$ shows up within the various functions composing $\Pi(\cdot)$, because these functions could vary across agents. For example, different agents may be endowed with different cost functions.

${ }^{3}$ Let $\mathbf{T}$ be the set of all functions $t: I \rightarrow \mathbb{R}_{+}$, i.e., $\mathbf{T}$ is the set of all action profiles. A given aggregator function $Z$ is a mapping $Z: \mathbf{T}_{Z} \rightarrow \mathbb{R}_{+}$defined on a subset $\mathbf{T}_{Z} \subset \mathbf{T}$. An action profile is ( $Z$-)viable if $t \in \mathbf{T}_{Z}$. For example, if $Z(t) \equiv \int_{I} t(i) d i$, then $\mathbf{T}_{Z}$ consists of all (Riemann)-integrable functions $t: I \rightarrow \mathbb{R}_{+}$.
} 
directly in market shares, rather than indirectly choosing them via $t(i)$. This approach delivers a direct and powerful way of analyzing how $x$ affects the equilibrium market (or payoff) shares.

To be precise, let the market share function $p(i, t, T ; x)$ in $(2)$ be $t$-bijective for any given $T>0, x \in X$ and $i \in I$. Then, any chosen action $t(i) \in \mathbb{R}_{+}$has a unique number $p(i) \in \mathbb{R}_{+}$ associated with it for given $(i, T, x)$. By a change-of-variable, we rewrite $(2)$ as

$$
\Pi(i, p(i), T ; x)=p(i) V(i, p(i), T ; x)-\Phi(i, p(i), T ; x)
$$

with some (convenient) abuse of notation $\sqrt[4]{4}$ We now define an equilibrium as a situation, where each agent $i$ directly chooses her market share $p(i) \in \mathbb{R}_{+}$as to maximize the payoff function (4), and all market shares must add up to one.

Definition 1 (Equilibrium) An equilibrium is a pair $(p(\cdot), T)$ consisting of a market share function $p:[0,1] \rightarrow \mathbb{R}_{++}$and a number $T \in(0, \infty)$ such that

i) For each $i \in I, p(i)$ solves $\max _{p(i) \geq 0} \Pi(i)$, where $\Pi(i)$ is given by (4).

ii) $\int_{I} p(i) d i=1$

Whenever $(p(i), T)$ is an equilibrium as in Definition 1 , then the unique actions $t(i)$ corresponding to the equilibrium market shares $p(i)$ together with $T=Z(t)$ must be an equilibrium in the original sense, and vice-versa, as a consequence of the bijective relation between actions and market shares 5 Further, we restrict attention to interior equilibria, where each agent obtains a positive equilibrium market share.6

What makes the transformed version of the model convenient in the context of our inequality analysis is that it will lead to a simple, type-recursive equilibrium equation identifying the inequality effects caused by $x$ without the need to solve the model for $t(i)$ or $T]^{7}$

Continuum Agents A final remark concerns the use of "continuum" agents. We show in Appendix B.3 that continuum agents are without loss of generality in such that, for a given number of atomistic agents $n \in \mathbb{N}$, the "discrete" equilibrium market shares $p^{d}(i)$ can be identified from the corresponding equilibrium (step-)density function $p(i)$ with support $[0,1]$ by rescaling the latter 8 These steps essentially represent the different agent types as specified by the ex ante agent heterogeneity (see below). One formal advantage of working with a continuum is that the equilibrium $p(\cdot)$ is a (Lebesgue) density $p:[0,1] \rightarrow \mathbb{R}_{+}$with a fixed support for any number of agent types, rather than a discrete mapping with variable support depending on the

\footnotetext{
${ }^{4}$ If $p=p(i, t, T)$ then $t \equiv p^{-1}(i, p, T)$ is the inverse of the function $p(i, t, T)$ with respect to the variable $t$. Hence $\Pi(i, t(i), T)=\Pi\left(i, p^{-1}(i, p(i), T), T\right) \equiv \hat{\Pi}(i, p(i), T)$. The abuse of notation is that we continue to use the notation $\Pi(i, p(i), T)$ (instead of $\hat{\Pi}(\cdot))$ in the transformed problem (similarly, we use $V$ and $\Phi$ instead of $\hat{V}, \hat{\Phi}$ ).

${ }^{5}$ We prove this claim in Appendix B.1.

${ }^{6}$ This is not too restrictive as we consider a fixed set of agents. It is straightforward to include agent entry or exit in our approach; see Hefti and Lareida (2020) for an application.

${ }^{7}$ Because the "direct" and "indirect" approach are equivalent, one can always try to infer how $p(i)$ depends on $x$ using the indirect approach via $t(i)$. However, this procedure frequently turns out to be less tractable for our purpose.

${ }^{8}$ For example, if $n=3$ and $p^{d}(1)=1 / 2, p^{d}(2)=1 / 3, p^{d}(3)=1 / 6$, then $p(i)=3 / 2, i \in[0,1 / 3), p(i)=1$, $i \in[1 / 3,2 / 3)$ and $p(i)=1 / 2, i \in[2 / 3,1]$, and $\int p(i) d i=1 / 3(3 / 2+1+1 / 2)=1$.
} 
number of agents.9 $9^{9}$ Moreover, this formulation includes the case of "true continuum agents", which basically means that every agent $i$ is a unique type.

\subsection{Regularity Assumptions}

Studying the distribution of market shares and related inequality measures is challenging, and we need to impose certain regularity conditions in order to bring the analytical complexity to a tractable level. Let $g(i, p, T ; x) \equiv \frac{\partial}{\partial p} p V(i, p, t ; x)$ and $\varphi(i, p, T ; x) \equiv \frac{\partial}{\partial p} \Phi(i, p, T ; x)$, where $g(\cdot)$ can be interpreted as marginal benefits and $\varphi(\cdot)$ as marginal costs, respectively, pertaining to an aspired market share $p$. For given $i$ and $T>0$, the FOC from maximizing (4) at an interior point $p(i)>0$ then amounts to the well-known "marginal benefits equal marginal costs":

$$
g(i, p(i), T ; x)=\varphi(i, p(i), T ; x)
$$

or, in short-hand notation, $g(i)=\varphi(i)$.

Assumption 1 (Regularity) We impose the following technical assumptions:

(A1) For any $x \in X, T>0, i \in[0,1]$ and $p \geq 0$ : $\Pi(i)$ is a strongly quasiconcave $C^{2}$ function of $p, g(i, 0, T ; x)>0$ and $g(i, \cdot, T ; x)$ is bounded from above, $\varphi_{p}(i, p, T ; x)>0$, $\varphi(i, 0, T ; x)=\Phi(i, 0, T ; x)=0$, and $\varphi(i, p, T ; x) \stackrel{p \rightarrow \infty}{\longrightarrow} \infty$.

(A2) For any $i \in[0,1]$ and $x \in X: g(i, p, \cdot ; x)$ and $\varphi(i, p, \cdot ; x)$ are $C^{1}$-functions, $g(i, 1,0 ; x)>0$, $g(i, 1, \cdot ; x)$ is bounded from above, $\varphi(i, p, 0 ; x)=0, \varphi_{T}(i, p, T ; x)>0$ and $\varphi(i, p, T ; x) \stackrel{T \rightarrow \infty}{\longrightarrow}$ $\infty$ if $p>0$, and $g_{T}(i, p, T ; x)<\varphi_{T}(i, p, T ; x)$ whenever $g(i, p, T ; x)=\varphi(i, p, T ; x)$.

(A3) For any $i \in[0,1]$ and any $p, T>0, g(i, p, T ; x)$ and $\varphi(i, p, T ; x)$ are $C^{1}$ in $x$.

The boundary and slope assumptions (A1) and (A2) jointly assert sufficiency of (5) with respect to the optimization problem in Definition 1, and the existence of a unique equilibrium which, for the sake of completeness, we prove in Appendix B.2. Assumption (A3) allows the use of calculus to study the inequality effects. Note that (A3) includes the case where $g(i)$ or $\varphi(i)$ do not depend on $x$.

\subsection{Agent Heterogeneity}

If different agents have different payoff functions, the market shares will not be uniformly dispersed. We now make the notion of ex ante agent heterogeneity precise.

Assumption 2 (Order) For any $p, T>0$ and any $x \in X: p V(i, p, T ; x), g(i, p, T ; x)$ are (weakly) decreasing and $\Phi(i, p, T ; x), \varphi(i, p, T ; x)$ (weakly) increasing in $i$.

This assumption implies that agents are sorted left-to-right, in thus that agent $i$ never features lower (marginal) benefits and never higher (marginal) costs than agent $j$ whenever $j>i$. A

\footnotetext{
${ }^{9}$ Nevertheless, our inequality tools apply to the case of finitely many atomistic agents, with the natural adjustments in the definitions. Specifically, condition $\int p(i) d i=1$ in Defintion 1 is replaced by $\sum_{i=1}^{n} p(i)$, and $p(i)$ is a density with respect to the counting measure.
} 
simple example is if agents may differ in their ability to compete for an object of identical value to all agents, such that

$$
\Pi(i)=p(i) V(p(i), T)-c(i) \Phi(p(i), T),
$$

where $c(i)$ is increasing in $i$. Taken together, Assumptions 1 and 2 imply that agents with a lower index $i$ achieve larger market shares and or payoffs 10

Proposition 1 (Equilibrium Order) Let $(p(\cdot), T)$ be an equilibrium for a given $x \in X$, and consider any two agents with $j>i$. Then under Assumptions 11 and 2:

i) No "leap-frogging": $p(i) \geq p(j)$ and $\Pi(i) \geq \Pi(j)$.

ii) Strict order: $p(i)>p(j)$ if $g(i, p, T) \geq g(j, p, T)$ and $\varphi(i, p, T) \leq \varphi(j, p, T) \forall p, T>0$ and at least one strict inequality, and $\Pi(i)>\Pi(j)$ if $p V(i, p, T) \geq p V(j, p, T)$ and $\Phi(i, p, T) \leq$ $\Phi(j, p, T) \forall p, T>0$ and at least one strict inequality.

iii) Equality: $p(i)=p(j)$ if both $g(i, p, T)=g(j, p, T)$ and $\varphi(i, p, T)=\varphi(j, p, T) \forall p, T>0$.

Property i) follows from Assumption 2 and individual optimality: If $p(i)<p(j)$, then the ex ante stronger agent $i$ could always benefit from deviating to the market level $p(j)$, for which reason there can be no such deviations in equilibrium. The other claims have similar reasons.

\subsubsection{Agent Types}

By ii) and iii) of Proposition 1, the equilibrium market share function reflects the order structure imposed by the ex ante agent heterogeneity. In the following, we consider the two order structures we deem most relevant: The case of finitely many different agent types as well as the case of a proper continuum of agent types. To clarify, consider example (6) and suppose that $c(\cdot)>0$ belongs to one of the following two classes:

- Class I : $\Leftrightarrow$ All increasing, right-continuous step functions for which $\exists i_{0} \in(0,1): i<i_{0} \leq j$ $\Rightarrow c(i)<c(j), i, j \in[0,1]$.

- Class II $: \Leftrightarrow$ All strictly increasing functions $c \in C^{1}([0,1],[1, \bar{c}])$.

It is evident from ii) and iii) in Proposition 1 that the equilibrium density $p(\cdot)$ must reflect the order structure of the respective class. E.g., if $c(\cdot)$ belongs to Class I, then $p(\cdot)$ must be a rightcontinuous step function in equilibrium; see Figure 1 for an illustration. Such cost functions matter, because they are the continuum analogue to the case of finitely many different cost types (see Appendix B.3); the different steps of $c(\cdot)$ represent the different types 11 Likewise, $p(\cdot)$ is a density with $p^{\prime}(i)<0$ on $(0,1)$ if $c(\cdot)$ is Class II. Note that both classes exclude, by their definitions, the case where all agents have the same value of $c(i)$, in which case all agents were perfect clones.

\footnotetext{
${ }^{10}$ Assumption 2 is stronger than what we actually require. We only need that the equilibrium order of the market shares is not pivoted by the parameter $x$, i.e., if $p(i ; x) \geq p(j ; x)$ then also $p\left(i ; x^{\prime}\right) \geq p\left(j ; x^{\prime}\right) \forall x^{\prime} \in X$ and any $i, j$. Then, ordering the agents left-to-right is without loss of generality.

${ }^{11}$ Specifically, as Figure 1 shows, if $c(\cdot)$ is Class I, i.e., a step function capturing agent cost types $k=1, \ldots, K$ with measures $\gamma_{1}, \ldots, \gamma_{K}>0, \sum_{k} \gamma_{k}=1$, then $p(\cdot)$ must be a corresponding step-wise decreasing density, meaning that $p(I)=\left\{p\left(i_{1}\right), \ldots, p\left(i_{K}\right)\right\}$ and $\int_{0}^{1} p(i) d i=\sum_{k=1}^{K} \gamma_{k} p\left(i_{k}\right)$.
} 

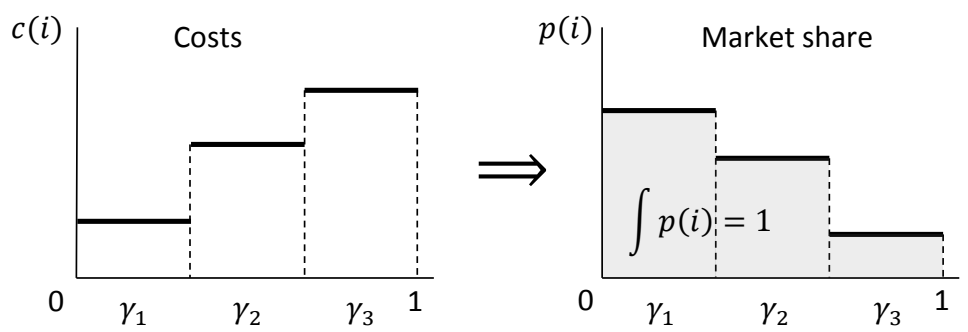

Figure 1: Costs and market share functions for $K=3$ (Class I)

It is straightforward to extend the logic of the above example to other forms of ex ante agent heterogeneity ${ }_{12}^{12}$ Whenever the agents differ such that there are finitely many different payoff functions, the equilibrium density $p(\cdot)$ must be a downward-stepping density as in Figure 1 under Assumptions 1 and 2, where the steps of $p(\cdot)$ amount to the different ex ante agent types. In what follows, we shall assume the ex ante agent heterogeneity to be such that the equilibrium $p(\cdot)$ is either a step function or a strictly decreasing $C^{1}$-function of $i$.

Definition 2 Let $p:[0,1] \rightarrow \mathbb{R}_{++}$be a density function. Then

- $p(\cdot)$ belongs to Class I if $p(\cdot)$ is a step-wise decreasing, right-continuous with at least one and at most finitely many downward steps.

- $p(\cdot)$ belongs to Class II if $p(i)$ is a $C^{1}$-function with $p^{\prime}(i)<0$ for $i \in[0,1]$.

Note that if $p(\cdot)$ belongs either to Class I or II, then $p(\cdot)$ must display the "somewhere strictly decreasing" (SSD) property: $\exists i_{0} \in(0,1): p(i)>p(j)$ for $i<i_{0} \leq j$. Further, the CDF associated with $p(\cdot)$ must be increasing, concave and strictly above the diagonal for $i \in(0,1)$ if $p$ is of Class I or II (see Appendix B.4.3).

Let $p(\cdot)$ be an equilibrium density. Then $[i] \equiv\{s \in[0,1]: p(s)=p(i)\}$ is the equivalence class of agents with representative $i \in I$. By Proposition 1, the agents within a given equivalence class are endowed with identical payoff functions. In this sense we think of $i$ as the type of all agents in $[i]$ :

Definition 3 (Agent Type) Let $p:[0,1] \rightarrow \mathbb{R}_{++}$be a decreasing density, and for any $i \in$ $[0,1]$ let $[i] \equiv\{s \in[0,1]: p(s)=p(i)\}$. Then, the collection of these equivalence classes on $[0,1]$ corresponds to the set of agent types, and all agents in a given equivalence class have the same type. Further, we define a transitive relation $\triangleright$ by $j \triangleright i: \Longleftrightarrow j>i$ and $j \notin[i]$.

The relation $\triangleright$ allows for an easy reference in terms of different agent types. If $p(\cdot)$ is Class I, $j \triangleright i$ means that $j$ is of a "weaker" type than $i$, i.e., $j$ sits on a lower step of $p(\cdot)$ than $i$. If $p(\cdot)$ is of Class II, the relations " $\triangleright$ " and ">" coincide.

\footnotetext{
${ }^{12}$ For example, it immediately follows that whenever the agent heterogeneity is such that $I=[0,1]$ can be partitioned by a finite number of intervals $I_{1}, \ldots, I_{n}$ of $n>1$ distinct agent groups consisting of homogeneous members, such that $g(i, p, T) \geq g(j, p, T)$ and $\varphi(i, p, T) \leq \varphi(j, p, T)$, one inequality strict, whenever $j>i, i \in I_{n}$ but $j \notin I_{n}$, then $p(\cdot)$ must be a step-wise decreasing density with finitely many steps, where $p(i)=p(j)$ iff $i, j \in I_{n}$.
} 


\section{Inequality Effects}

We say that the parameter $x$ induces inequality effects if the equilibrium market share function $p(\cdot)$ depends on $x$.

Definition 4 (Inequality Effects) Given a competition for market shares $(\{\Pi(i)\}, Z)$, the parameter $x$ induces inequality effects if $\exists x, x^{\prime} \in X: p(\cdot ; x) \neq p\left(\cdot ; x^{\prime}\right)$ for the respective equilibrium market share functions. If $p(\cdot ; x)=p\left(\cdot ; x^{\prime}\right) \forall x \in X$, then $x$ is inequality preserving.

With respect to the possible inequality effects, we focus on those that affect the equilibrium $p(\cdot ; x)$ in a way that preserves the agent order. To state this formally, let $p(\cdot ; x)$ denote the equilibrium market share function for any $x \in X$. For each $i$ and any $x \in X$, let $[i]_{x} \equiv$ $\{s \in[0,1]: p(s)=p(i)\}$ be the set of $i$-equivalent agent types in equilibrium, such that $\mathcal{T}_{x} \equiv\left\{[i]_{x}: i \in[0,1]\right\}$ is the equilibrium set of agent types for a particular $x$. Note that under Assumptions 1 2, a unique equilibrium market share function $p(\cdot ; x)$ exists $\forall x \in X$, such that the assignment $x \mapsto \mathcal{T}_{x}$ is well-defined. Our notion of an order-preserving market condition means that this assignment is constant.

Assumption 3 (Order-Preserving Market Conditions) $x$ is an order-preserving market condition if $\mathcal{T}_{x}=\mathcal{T}_{x^{\prime}}, \forall x, x^{\prime} \in X$, such that all $x \in X$ generate the same set of types in equilibrium, and $p(\cdot ; x)$ is weakly decreasing $\forall x \in X$.

If $x$ is an order-preserving market condition, then the agent ordering as reflected by the equilibrium market share function $p(\cdot)$ is unaffected by $x$. That is, if agent $i$ obtains a larger equilibrium market share than $j$ for $x$, then the same holds for any other $x^{\prime} \in X[13$ Note that the definition of an order-preserving market condition does not exclude that $x$ could affect only a single agent, i.e., is an idiosyncratic rather than a common condition. 14 We only require that changes in $x$ cannot induce a leap-frogging of one or several agents. This can always be assured, e.g., if $p(\cdot)$ is Class I and the changes in $x$ remain sufficiently small.

We are particularly interested in the case where $x$ is a common market condition, meaning that $x$ affects marginal benefits or marginal costs of each agent in a qualitatively similar manner, while respecting the agent order.

Definition 5 (Common Market Condition) An order-preserving market condition $x$ is a common market condition if for any given $p, T>0 g(i, p, T ; x)$ and $\varphi(i, p, T ; x)$ either increase, decrease or are constant in $x$ for all agents.

As an example, suppose that an increase in $x$ increases marginal benefits for each agent, i.e., $g_{x}(i)>0 \forall i$, implying that all agents aspire a larger market share. This effect, however, can be differentially strong for various agents, making it generally an open and non-trivial question how the market or payoff share distributions settle down in the new equilibrium.

\footnotetext{
${ }^{13}$ If $p(\cdot ; x)$ is Class I, this means that $p\left(\cdot ; x^{\prime}\right)$ remains a Class I density $\forall x^{\prime} \in X$, where the length of each step is invariant to $x^{\prime}$ (capturing that agent types remain the same) and the steps go down for any given $x$.

${ }^{14}$ For example, if $g_{x}(i) \neq 0$ or $\varphi_{x}(i) \neq 0$, while $g_{x}(j)=\varphi_{x}(j)=0 \forall j \neq i$ this amount to a purely idiosyncratic condition, where only agent $i$ is directly affected by (a change) in $x$.
} 
Structure of Section 3 In Section 3.1 we derive a single type-recursive equation from the optimality condition (5) which is the basis of our inequality analysis. This equation can be decomposed into a direct-aggregative and an indirect effect of $d x$. The former effect alone is decisive for whether inequality effects arise for any conceivable marginal change $d x$ that affects one, many or all agents (Theorem 1). We exemplify the direct-aggregative effects if $x$ is a "level variable", which is a relevant concept for many of our later applications.

In Section 3.2 we focus on rotations of the market share function as a specific type of inequality effect, and prove that, again, the direct-aggregative effect alone determines whether the inequality effects take on the form of a rotation (Theorem 2). The indirect effect determines additional properties of rotations (Proposition 2), such as whether $x$ has a monotonic effect on inequality (Theorem 4). The latter is likely to occur if marginal costs and benefits can be represented as power functions (Proposition 3).

Finally, we present a procedure for determining the comparative-statics of the aggregate $T$ based on our formulation as a competition for market shares. Knowing $\operatorname{sign} T^{\prime}(x)$ turns out to be relevant for some of our inequality theorems.

Throughout the rest of Section 3, we take Assumptions 1, 3 as satisfied, and suppose that the ex ante heterogeneity is such that $p(\cdot)$ belongs to Class I or II.

\subsection{Existence of inequality effects}

Let $x_{0} \in X$. As (5) holds $\forall i$ in equilibrium, it follows that equilibrium forces equate the ratio of marginal benefit over marginal costs for any two agents. Hence

$$
\frac{g\left(i, p(i), T ; x_{0}\right)}{\left.\varphi(i, p(i), T) ; x_{0}\right)}=\frac{g\left(j, p(j), T ; x_{0}\right)}{\left.\varphi(j, p(j), T) ; x_{0}\right)} \quad i, j \in[0,1]
$$

or, in short-hand, $g(i) / \varphi(i)=g(j) / \varphi(j)$. We exploit (7) to obtain a simple, type-recursive relation that characterizes the inequality effects induced by a marginal change $d x$. The following definitions are required:

$$
d p(i) \equiv \frac{\partial p\left(i ; x_{0}\right)}{\partial x}, \quad \Delta_{i} \equiv \frac{d p(i)}{p(i)}, \quad \varepsilon_{i} \equiv \frac{g_{p}(i) p(i)}{g(i)}, \quad \eta_{i} \equiv \frac{\varphi_{p}(i) p(i)}{\varphi(i)} .
$$

Thus, $\Delta_{i}$ is the percentage change in equilibrium market share of agent $i$, and $i$ gains market share if and only if $\Delta_{i}>0$. Further, $\varepsilon_{i}, \eta_{i}$ are the elasticity of marginal benefits $g(\cdot)$ and costs $\varphi(\cdot)$, respectively, with respect to $p$.

Lemma 1 Let $j \triangleright i$, and consider a marginal change $d x>0$. Then $\eta_{i}>0, \varepsilon_{i}<\eta_{i}$, and

$$
\begin{gathered}
\Delta_{i}=\Delta_{j} k_{i j}+R_{i j}, \quad k_{i j} \equiv \frac{\eta_{j}-\varepsilon_{j}}{\eta_{i}-\varepsilon_{i}}>0, \\
R_{i j}=\frac{A(i)-A(j)}{\underbrace{\eta_{i}-\varepsilon_{i}}_{>0}}, \quad A(s) \equiv\left(\frac{g_{T}(s)}{g(s)}-\frac{\varphi_{T}(s)}{\varphi(s)}\right) T^{\prime}\left(x_{0}\right)+\left(\frac{g_{x}(s)}{g(s)}-\frac{\varphi_{x}(s)}{\varphi(s)}\right) .
\end{gathered}
$$

The type-recursive equation (9) decomposes the relation between $\Delta_{i}$ and $\Delta_{j}$ into a directaggregative effect $\left(R_{i j}\right)$, and an indirect effect $\left(k_{i j}\right)$. To understand these names, note that $R_{i j}$ 
depends directly on $x$ via $g_{x}(s)$ and via the aggregate quantity $T^{\prime}(x)$. By contrast, $k_{i j}$ collects the indirect effects that $d x$ has on $g(\cdot)$ and $\varphi(\cdot)$ via the changes in $p(i)$. We shall often write $R$ and $k$ if there is no confusion about types.

Decomposition (9) is key for analyzing if and how $x$ affects $p(i)$. The first theorem shows that inequality effects exist if and only if the direct-aggregative effect $R_{i j}$ is non-zero for at least two different agent types.

Theorem 1 (Existence of inequality effects) If $R_{i j}=0 \forall i, j \in[0,1]$ and any $x \in X$, then $x$ is inequality preserving. Conversely, if for a given $x \in X \exists i, j \in[0,1]$ such that $R_{i j} \neq 0$, then inequality effects arise. Specifically, $\exists \delta>0$ such that $p\left(\cdot ; x^{\prime}\right) \neq p(\cdot ; x)$ for any $x^{\prime} \in$ $(x-\delta, x+\delta) \backslash\{x\}$.

Theorem 1 characterizes the existence of inequality effects independent of whether $d x$ affects all or only some agents. By Theorem 1, the indirect effect $k_{i j}$ plays no role for whether inequality effects arise, which is fairly intuitive. The indirect effect captures how sensitive $g(\cdot), \varphi(\cdot)$ respond to changes in $p$, and as such may influence certain quantitative aspects should inequality effects occur, but not whether they arise in the first place.

By Theorem 1, $x$ is inequality preserving if and only if $A(\cdot)$ is invariant to the agent index $i$ $\forall x \in X$. If $x$ affects the agents in a differential way, e.g., by entering the payoffs of some but not all agents, then inequality effects most likely arise. By contrast, one could expect $x$ to be inequality preserving if $x$ affects all agents in a similar manner. This leads us to the notion of a level variable.

\subsubsection{Level Variables}

Suppose, for simplicity, that $x$ affects only $g(i)$, the RHS of (5), for each agent ${ }^{15}$ We then say that $x$ is a level variable if $x$ affects $g(i)$ by the same proportion for all agents.

Definition 6 (Level Variable) A common market condition $x$ is a level variable if $\frac{g_{x}(i)}{g(i)}$ and $\frac{g_{T}(i)}{g(i)}$ in (5) are independent from $i$ and $p(i) \forall x \in X, \forall T>0$.

Note that the definition of a level variable includes both the direct and the aggregative effect caused by a change of $x \sqrt{16}$ Further, $x$ is a level variable if and only if $g(i)$ is multiplicative separable in $(i, p)$ and $(T, x){ }^{17}$ A special case occurs if marginal benefits are identical for all agents, i.e., $g\left(i, p_{i}, T ; x\right)=V(T ; x)$, such that (5) becomes

$$
V(T ; x)=\varphi(i, p(i), T)
$$

This amounts to the case of a symmetrical competition, in thus that the benefit per unit of market share, $V(T, x)$, is identical for all agents.

In $A(i)-A(j)$ from $(10)$, the terms $\frac{g_{x}(i)}{g(i)}-\frac{g_{x}(j)}{g(j)}$ and $\frac{g_{T}(i)}{g(i)}-\frac{g_{T}(j)}{g(j)}$ cancel $\forall i, j$ and all $x \in X$ if and only if $x$ is a level variable. Thus, with level variables, the inequality effects essentially

\footnotetext{
${ }^{15}$ It is straightforward to adjust Definition 6 if $x$ enters $\varphi(i)$ instead.

${ }^{16}$ Formally, we have that $\frac{\partial g(i)}{\partial x}=g_{x}(i)+g_{T}(i) T^{\prime}(x)$.

${ }^{17} \mathrm{~A}$ function $f(i, p, T, x)$ is multiplicatively separable in $(i, p)$ and $(T, x)$ if $f(i, p, T, x)=u(i, p) v(T, x)$. Note that multiplicative separability includes the trivial case where $f(i, p, T, x)$ does not depend, e.g., on $T$ at all.
} 
boil down to how the aggregate $T$ affects marginal costs for the different agents. The following result makes this explicit.

Corollary 1 The level variable $x$ is inequality preserving if $\varphi(i)$ in (5) is multiplicatively separable in $(i, p)$ and $T$. Conversely, if $x$ is inequality preserving and $T^{\prime}(x) \neq 0 \forall x \in X$, then marginal costs must be multiplicatively separable in $(i, p)$ and $T$.

This result is intuitive. For if $\varphi(i)$ is multiplicatively separable in $(i, p)$ and $T$, then marginal costs are affected by the same proportion $\forall i$ by a change of $T$. This counterbalances that marginal benefits change in proportion, resulting in a stable market share distribution.

If the market share is of the form $p(i)=t(i) / T$, such that $t(i)=p(i) T$, we can say more about whether a level variable induces inequality effects.

Corollary 2 Let $p(i)=t(i) / T$. A level variable is inequality preserving if $\Phi(i, t)=c(i) t^{\gamma}$. If $T^{\prime}(x) \neq 0 \forall x \in X$ and the level variable is inequality preserving, then $\Phi(i, t)=c(i) t^{\gamma}$.

Under the respective requirements, Corollary 2 shows that it is necessary and sufficient for a level variable to be inequality preserving that costs are power functions with an identical exponent. This points to a decisive role of power functions, and one that we shall explore further in the next sections.

The requirement that $T^{\prime}(x) \neq 0$ in Corollaries $1-2$ is weak and satisfied in all our applications. By Corollary 1 it therefore depends exclusively on $\varphi(i)$ whether inequality effects arise or not in case of level variables. In Appendix B.4.2 we pursue the opposite reasoning, and study the inequality effects that can arise due to a change in a common market condition given that $\varphi$ is of the separable form $\varphi(i)=c(i, p(i)) C(T)$.

\subsection{Rotations}

Given that $x$ induces inequality effects, can more be said about their properties? The following section provides an affirmative answer by considering the possibility that $x$ induces a rotation of $p(\cdot)$.

Definition 7 (Rotations) Let $x, x^{\prime} \in X$ and $p(\cdot, x), p\left(\cdot, x^{\prime}\right)$ be two decreasing densities with support $[0,1]$. We say that $p\left(\cdot ; x^{\prime}\right)$ is an outward-rotation $(\boldsymbol{O R})$ of $p(\cdot ; x)$, or $p(\cdot ; x)$ is an inward-rotation (IR) of $p\left(\cdot ; x^{\prime}\right)$, if $\exists 0<i_{0} \leq i_{1}<1$ such that

$$
\begin{array}{ll}
p\left(i ; x^{\prime}\right)>p(i ; x) & i \in\left[0, i_{0}\right) \\
p\left(i ; x^{\prime}\right)<p(i ; x) & i \in\left(i_{1}, 1\right) \\
p\left(i ; x^{\prime}\right)=p(i ; x) & i \in\left(i_{0}, i_{1}\right]
\end{array}
$$

where the last condition only applies if $i_{0}<i_{1}$. We say that a parameter change $d x>0$ induces an $O R(I R)$ of $p(\cdot ; x)$ if $\exists \delta>0$ such that $p\left(\cdot ; x^{\prime}\right)$ is $O R(I R)$ of $p(\cdot ; x)$ for any $x^{\prime} \in(x, x+\delta)$.

The defining property of a rotation is that there is a "turning point", where the market shares of all stronger agents increases (decreases) while it decreases (increases) for all weaker agents (see Figure 2). 


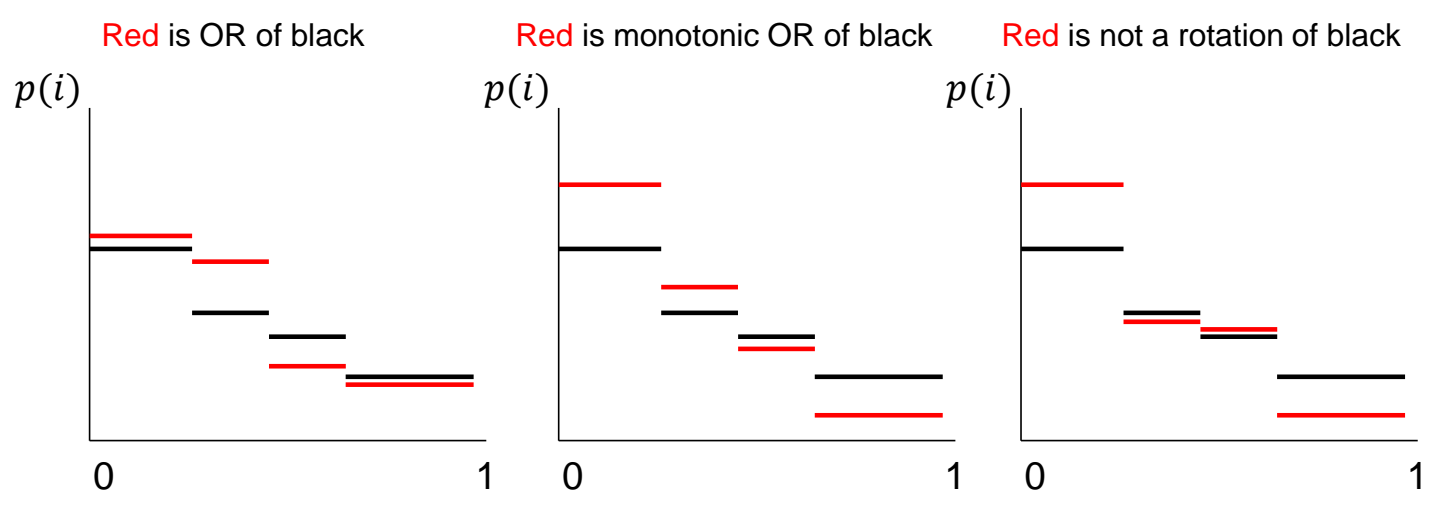

Figure 2: Class I rotations: Examples and counterexample

Lorenz Dominance If $p\left(\cdot, x^{\prime}\right)$ is an OR (IR) of $p(\cdot, x)$, then the dispersion of market shares has become less (more) equal. This is related to the Lorenz curve, which is a common tool in inequality analysis. A distribution Lorenz dominates another distribution if its Lorenz curve lies below the Lorenz curve of the other. Many standard inequality measures, such as the Gini coefficient or the notion of first-order stochastic dominance, are consistent with the partial order generated by Lorenz dominance (Atkinson, 1970). The rotation-order ranks two market share functions according to first-order stochastic dominance (see Appendix B.4.3). Thus, if $p\left(\cdot ; x^{\prime}\right)$ is an OR (IR) of $p(\cdot ; x)$, then the former Lorenz dominates (is Lorenz dominated by) the latter. It follows that $p\left(\cdot ; x^{\prime}\right)$ features less (more) equality than $p(\cdot ; x)$ according to any Lorenz-consistent inequality measure.

\subsubsection{Existence of Rotations}

The following theorem shows that the direct-aggregative effect $R$ alone is decisive for whether a rotation arises.

Definition $8 R$ is uniformly positive (negative) at $x_{0} \in X$, if $R_{i j}\left(x_{0}\right)>(<) 0 \forall j \triangleright i$. If $R$ is uniformly positive (negative) $\forall x \in X$, then $R$ is globally uniformly positive (negative).

Theorem 2 (Rotational Effects) If $p(\cdot)$ belongs to Class I and $R$ is uniformly positive (negative) at $x_{0} \in X$, then $d x>0$ always induces an $O R(I R)$ of $p\left(\cdot, x_{0}\right)$. If $p(\cdot)$ belongs to Class $I$ or II and $R$ is globally uniformly positive (negative), then the market shares of the strongest types $i \in[0]$ increase (decrease) strictly in $x$, while the ones of the weakest types $i \in[1]$ strictly decrease (increase).

To understand the first result, note that $A(i)$ captures how sensitively marginal costs and benefits respond to $x$ and $T$, which amount an agent's incentives to adjust her market share. If $A(i)>A(j), j \triangleright i$, such that $R_{i j}>0$, then stronger types face stronger incentives to aspire a larger market share (or weaker incentives to reduce their market shares). This intuitively implies that whenever type $i$ manages to increase her equilibrium market share, this holds for any stronger type as well. Because not all market shares can increase, the resulting inequality effect takes on the form of a rotation. 
The second result shows that the tails of the distribution evolve monotonically in $x$ under the respective conditions. A corollary to this result is that the inequality effects in the two- or three-types cases are fully characterized 18

Theorems 1 and 2 show that the local behavior of the equilibrium function (5) is highly informative about the global properties of the equilibrium distribution $p(\cdot)$. The uniformity requirement on $R$ is met by all our applications in Section 4 (if $R \neq 0$ ), and thus appears not to be restrictive in the context of common market conditions. More generally, one could seek to identify structural conditions assuring that $R>(<) 0$ uniformly. We pursue this next in case of level variables, and in Appendix B.4 for the case of multiplicative separable cost functions.

Level Variables We now evaluate Theorem 2 if $x$ is a level variable and $p(\cdot)$ is Class I. Then, sign $R$ is only determined by the properties of the marginal costs $\varphi(i)$. Moreover, by using the elasticity function

$$
\theta(i) \equiv \frac{\varphi_{T}(i) T}{\varphi(i)}
$$

condition 10 yields that $\operatorname{sign} R_{i j}=\operatorname{sign}(\theta(j)-\theta(i)) T^{\prime}(x)$. This shows that the elasticity of marginal costs $\varphi(i)$ provide a sufficient criterion for a level variable to induce a rotation.

Corollary 3 Let $x$ be a level variable and $T^{\prime}(x)>0$. If $\theta(i)<(>) \theta(j) \forall j \triangleright i$, then $R_{i j}>(<) 0$ and $x$ induces an OR (IR) of $p(\cdot)$.

Corollary 3 equally applies for $T^{\prime}(x)<0$ if "OR" and "IR" are interchanged. Further, if $\theta(i)=\theta(j) \forall i, j$, then $\varphi(i)$ must be multiplicatively separable in $(i, p)$ and $T$, meaning that $x$ is inequality preserving (see Corollary 3 ).

The intuition in case of an OR is as follows. Let $j \triangleright i$, such that agent $j$ currently has a smaller market share than agent $i$, reflecting an ex ante advantage of agent $i$. An increase in a level variable induces the same incentive for all agents to expand their market shares, which is associated with higher expenses. If $\theta(i)<\theta(j)$, the marginal costs of the agent with the larger market share increases at a slower pace, meaning that this agent can adjust better to the new situation than its competitors with lower market shares. If such a ranking applies for any two different agent types, the agents with the largest market shares are affected least in terms of costs, explaining why an OR results.

If $p(i)=t(i) / T$, the elasticity condition in Corollary 3 can be stated directly in terms of the elasticity of the marginal cost function $\Phi_{t}(i, t) \equiv h(i, t)$. Let

$$
\psi(i) \equiv \frac{h_{t}(i, t) t}{h(i, t)}
$$

denote the $t$-elasticity of marginal costs $h(i, t)$. It then is easy to check that $\theta(i)=\psi(i)+1$, and thus:

\footnotetext{
${ }^{18}$ This follows because in the two-types case $R_{01} \geq(>) 0$ iff $R_{10} \leq(<) 0$, meaning that $R$ is either uniformly positive (negative) or $R=0$. Moreover, in the three-types case it follows from (the proof of) Theorem 2 that if $R$ is globally uniformly positive (or negative), then any $x>x_{0}$ induces an $\mathrm{OR}$ (IR) of $p\left(\cdot, x_{0}\right.$ ), because the behavior of the "middle group" does not matter.
} 
Corollary 4 Let $p(i)=t(i) / T$, and $x$ be a level variable with $T^{\prime}(x)>0$. If $\psi(i)<(>) \psi(j)$ $\forall j \triangleright i$, then $x$ induces an $O R(I R)$ of $p(\cdot)$.

\subsubsection{Monotonic Rotations}

Theorem 2 is useful for detecting rotations. However, a rotation is a fairly general type of inequality effect that is consistent with various patterns of the "middling" agents. For example, an OR can be consistent with some winning agents "catching up" with even stronger types. This is illustrated in Figure 2 (left panel), where among the winning agents the second strongest agents gain more market share than the strongest, such that the gap between these agents narrows. By contrast, the gap between both the winning and the losing agents widens in the rotation of the middle panel in Figure 2. The following definition makes the notion of a rotation featuring such "increasing gaps" precise.

Definition 9 (Monotonic Rotations) Suppose that $\infty>p\left(\cdot ; x^{\prime}\right), p(\cdot ; x)>0$ are right-continuous, decreasing SSD densities with the same equivalence classes [i]. If

$$
\frac{p\left(i ; x^{\prime}\right)}{p\left(j ; x^{\prime}\right)}>(<) \frac{p(i ; x)}{p(j ; x)} \quad \text { whenever } j \triangleright i \in(0,1)
$$

is satisfied, then $p\left(\cdot ; x^{\prime}\right)$ is a monotonic OR $(I R)$ of $p(\cdot ; x)$.

The fact that condition (15) actually induces a rotation is proved in Appendix B.4.1 19 Also note that Definition 9 applies to Class I and II market share functions ${ }^{20}$ In case of a monotonic rotation, the ratio of market shares is either increasing or decreasing as $x$ changes. This equivalently means that if $p\left(i ; x^{\prime}\right)$ is a monotonic OR (IR) of $p(i ; x)$, then the relative change in market shares is strictly increasing (decreasing) in agent type, such that the strongest agents $(i \in i[0])$ gain (lose) most while the weakest agents $(i \in i[1])$ lose (gain) most. Thus, a "catching up" as in Figure 2 (left panel) is impossible. This follows from the following fact.

Lemma 2 Consider real numbers with $u^{\prime}>u>0$ and $v^{\prime}>v>0$. If $\frac{u^{\prime}}{v^{\prime}} \geq \frac{u}{v}>1$, then also $u^{\prime}-v^{\prime}>u-v$.

If $p\left(\cdot ; x^{\prime}\right)$ is a monotonic OR of $p(\cdot ; x)$ and $i, j$ are two different agents types both featuring higher market shares in the new equilibrium, then the absolute gap between these market shares must have widened.

If 15 holds $\forall x \in X$, then $p\left(\cdot ; x^{\prime}\right)$ is a monotonic OR (or IR) of $p(\cdot ; x)$ for any $x^{\prime}>x 2^{21}$ Then, the rotations induced by $d x>0$ are transitive: If $p\left(\cdot ; x^{\prime \prime}\right)$ is a monotonic OR of $p\left(\cdot ; x^{\prime}\right)$ and $p\left(\cdot ; x^{\prime}\right)$ is a monotonic OR of $p(\cdot ; x)$, then $p\left(\cdot ; x^{\prime \prime}\right)$ must be a monotonic OR of $p(\cdot ; x)$, too (similarly for

\footnotetext{
${ }^{19}$ In general, Condition $\sqrt{15}$ is sufficient for a rotation to occur unless in the case of just two agent types, where 15], the rotation-property and stochastic dominance of the respective distribution functions are equivalent, which we prove in Appendix B.4.

${ }^{20}$ In the special case where $p(\cdot)$ is a Class II density and 15 holds for any $j>i, \sqrt{15}$ is known as the monotone likelihood property in mathematical statistics and econometrics (see, e.g., Casella and Berger (2002)). In economic theory, monotone likelihood ratios are sometimes imposed by mechanism design or contract theory as exogenous assumptions on the ex ante type distribution for reasons entirely unrelated to this article.

${ }^{21}$ If $p(\cdot ; x)$ is Class II and condition $(15)$ holds on $X$, this is equivalent to strict log-super(sub)modularity of $p(i ; x)$, but not if $p(\cdot ; x)$ is of Class I given that $p(\cdot)$ is a step function; Appendix B.4 discusses these claims in greater detail.
} 
IR). Thus, the relative market share $\frac{p(i ; x)}{p(j ; x)}, j \triangleright i$, is strictly increasing (decreasing) in $x$ with a monotonic OR (IR), meaning that market shares must be less and less equally (more and more equally) dispersed as $x$ increases. That is, the inequality of $p(\cdot ; x)$ must increase (decrease) over the entire parameter space $X$ as measured by any Lorenz-consistent inequality measure.

\subsubsection{Detecting Monotonic Rotations}

The aim of this section is to connect property (15) defining a monotonic rotation to some of our more primitive notions. The first result relates (15) to the $\Delta_{i}$-formulation in (8) which we used earlier to identify inequality effects.

Theorem 3 (Monotonic Rotations) Let $p(\cdot)$ be a Class I or II density, and $x_{0}, x \in X$. If

$$
\Delta_{i}(x)>\Delta_{j}(x) \quad \forall x \geq x_{0} \text { and any } j \triangleright i .
$$

then $p(i ; x)$ is a monotonic OR of $p\left(i ; x_{0}\right)$ for any $x>x_{0}$. If the first inequality in (16) is reversed, then $p(i ; x)$ is a monotonic IR of $p\left(i ; x_{0}\right)$ for any $x>x_{0}$.

Note that condition 16 is equivalent to

$$
\frac{\partial}{\partial x}\left(\frac{p(i ; x)}{p(j ; x)}\right)>0 \quad \forall x \geq x_{0} \text { and any } j \triangleright i .
$$

Theorem 3 is useful in applications, because it says that whenever we can infer condition 16 from the equilibrium equation (7), we may conclude that $p(\cdot ; x)$ is a monotonic OR (or IR) of $p\left(\cdot ; x_{0}\right)$ for any $x>x_{0}$, even if we cannot solve the equilibrium equation explicitely ${ }^{22}$ If (16) or (16 holds with equality instead of " $>$ " $\forall x$, then $x$ is inequality preserving, which yields a useful alternative test for the existence of inequality effects.

Corollary 5 Let $p(\cdot ; x)$ be a density function with support $[0,1]$ that is differentiable on $X$. Then, $x$ is inequality preserving if and only if $\frac{\partial}{\partial x}\left(\frac{p(i ; x)}{p(j ; x)}\right)=0 \forall i, j \in I$ and $\forall x \in X$.

In our previous results, we traced the existence of inequality effects and rotations back to the direct-aggregative effect $R$. We next show that it depends on the indirect effect $k$ whether a monotonic rotation results from a change of $x$.

We say that $k\left(x_{0}\right)$ is uniformly larger (smaller) than one at $x_{0}$ if $k_{i j}\left(x_{0}\right) \geq(\leq) 1 \forall j \triangleright i$. The following proposition shows that if $R$ is uniformly positive or negative, and $k$ is uniformly larger or smaller than one, the resulting rotation must display a certain quantitative pattern. In particular, the market shares of either the winners or losers must evolve monotonically, depending on which of the four possible cases arises.

Proposition 2 (Partially Monotonic Rotations) Let $p(\cdot ; x)$ be Class I, and suppose that $R$ is uniformly positive (negative) at $x_{0} \in X$. If $k\left(x_{0}\right)$ is uniformly larger than one, there is $\delta>0$ such that

$$
p\left(i ; x^{\prime}\right)>p(i ; x) \quad \Rightarrow \quad \frac{p\left(i ; x^{\prime}\right)}{p\left(i, x_{0}\right)}>(<) \frac{p\left(j ; x^{\prime}\right)}{p\left(j, x_{0}\right)} \quad \forall j \triangleright i
$$

\footnotetext{
${ }^{22}$ In Appendix $\mathrm{B} .4$ we derive an alternative rotation condition that operates over differences.
} 
for any $x^{\prime} \in\left(x_{0}, x_{0}+\delta\right)$. If $k$ is uniformly smaller than one, there is $\delta>0$ such that

$$
p\left(i ; x^{\prime}\right)<p(i ; x) \quad \Rightarrow \quad \frac{p\left(i ; x^{\prime}\right)}{p\left(i, x_{0}\right)}>(<) \frac{p\left(j ; x^{\prime}\right)}{p\left(j, x_{0}\right)} \quad \forall j \triangleright i
$$

for any $x^{\prime} \in\left(x_{0}, x_{0}+\delta\right)$.

In view of Definition 7, the conditions in Proposition 2 amount to partially monotonic rotations. If $R$ is uniformly positive, such that an OR results (Theorem 2), and $k$ is uniformly larger than one, (17) says that among winning agents, the stronger an agent is (lower index $i$ ), the more the agent gains in relative terms. Equivalently, if agent $i$ gains market share as $d x>0$ and $j \triangleright i$, then the relative market share $\frac{p(i ; x)}{p(j ; x)}$ must have strictly increased. By contrast, if $k \leq 1$ uniformly, then (18) says that among the losing agents, the weaker an agent is the more she loses. The same logic applies "from the other side" if $R<0$ uniformly, such that an IR results, and hence the weaker agents gain market shares while the stronger agents lose.

If $k$ is uniformly equal to one, both statements of Proposition 2 apply. The consequence, summarized by Theorem 4 , is that then $x$ must induce a monotonic rotation.

Theorem 4 (Monotonic rotations) Let $p(\cdot)$ be a Class I or II density. If $k(x)=1 \forall i, j \in$ $[0,1]$ and any $x \in X$, and $R$ is globally uniformly positive (negative), then $p(i ; x)$ is a monotonic OR (IR) of $p\left(i ; x_{0}\right)$ for any $x>x_{0} \in X$.

Intuitively, $k=1$ means that the marginal costs and benefits of all agents respond equally sensitive, ceteris paribus, to changes in their aspired market shares. Theorem 4 shows that in this case the mere uniform positivity of the direct-aggregative effect suffices to assure the existence of a monotonic rotation.

Level Variables If $x$ is a level variable, $p(i)=t(i) / T$, and $g(i)$ is multiplicatively separable in $i, p$ and $(T, x)$, we can express Proposition 2 in terms of cost elasticities (14). The reason is that in this case the indirect effect $k_{i j}$ is entirely determined by $\psi(i)$ and $\psi(j){ }^{23}$

Corollary 6 Let $p(i)=t(i) / T$, and $x$ be a level variable with $T^{\prime}(x)>0$. If $\psi(j)>(<) \psi(i)$ $\forall j \triangleright i$, then $R$ is uniformly positive (negative) and $k$ is uniformly larger (smaller) than one, such that a partially monotonic OR (IR) with property $(17)(\sqrt{18})$ ) results.

In terms of applications, Corollary 6 will be useful because it includes (11) as a special case.

\subsubsection{Power Functions}

The previous section shows that the indirect effect $k$ is decisive for whether a rotation is monotonic or not. The main result of this section shows that if $g(\cdot)$ and $\varphi(\cdot)$ in (5) can be written as general power functions, then $k=1$ uniformly and, accordingly, the rotations are monotonic.

Proposition 3 If $g(\cdot)$ and $\varphi(\cdot)$ in (5) are power functions of the form

$$
g(i, p, T ; x)=\hat{g}(i, T ; x) p^{\xi(T ; x)}, \quad \quad \varphi(i, p, T)=\hat{\varphi}(i, T) p^{\zeta(T)},
$$

\footnotetext{
${ }^{23}$ Multiplicative separability implies that $\varepsilon(i)=\varepsilon(j)$ in $(8)$, and $k_{i j}=\eta(j) / \eta(i)=\psi(j) / \psi(i)$.
} 
where $\xi(T ; x), \zeta(T)$ are real-valued functions, then $k(x)=1$ uniformly $\forall x \in X$. Thus, if $R$ is globally uniformly positive (negative), then $p\left(\cdot ; x^{\prime}\right)$ is a monotonic OR (IR) of $p(\cdot ; x) \forall x^{\prime}>x$.

Because the power function property is preserved under differentiation, 190 holds if costs and benefits in the payoff function $\Pi(\cdot)$ are power functions. Note that the conditions in 190 include the special cases where, e.g., $g(\cdot)$ does not depend on $p$ at all $(\xi(T ; x)=0)$, or where $\varphi(i, p, T)$ is linear in $p$. As we shall see in Section 4. many important examples in economics involve power functions and, by Theorem 4, can therefore are subject to strong inequality effects.

Some of our previous results on rotations were centered around how the ratio $p(i ; x) / p(j ; x)$ depends on $x$, ceteris paribus. These results are particularly convenient in case of power functions. If (19) applies, then

$$
\frac{p(i)}{p(j)}=\left(\frac{\hat{g}(i, T ; x)}{\hat{g}(j, T ; x)} \frac{\hat{\varphi}(j, T)}{\hat{\varphi}(i, T)}\right)^{\frac{1}{\zeta(T)-\xi(T ; x)}},
$$

and Theorem 3 tells us that $x$ induces a monotonic rotation whenever the RHS of 20 is strictly increasing in $x \forall j \triangleright i$ in equilibrium.

Level Variables If $p(i)=t(i) / T$ and costs are power functions $\Phi(i, t)=c(i) t^{\gamma_{i}}$, where $\gamma_{i}>0$ is constant for each $i$, then a stronger version of Corollary 4 results. In particular, given that $x$ is a level variable, the power function property (19) assures that $g(i)$ must be multiplicatively separable in $i, p$ and $(T, x)$, which implies that the rotations must be monotonic.

Corollary 7 Let $p(i)=t(i) / T, \Phi(i, t)=c(i) t^{\gamma(i)}$, and $x$ be a level variable with $T^{\prime}(x)>0$. If $\gamma_{i}<(>) \gamma_{j} \forall j \triangleright i$, then $x$ induces a monotonic OR (IR) of $p(\cdot)$. If $\gamma_{i}=\gamma \forall i, j$, then $x$ is inequality preserving.

\subsubsection{Payoff Inequality}

Up to now, our inequality analysis centered on the dispersion of market shares. We now consider inequality effects in the dispersion of payoff shares, and study the relation between market and payoff shares.

Let $s(i) \equiv \Pi(i) / \int_{I} \Pi(s) d s$ denote the payoff share earned by agent $i$. A fairly natural questions to ask is whether payoff shares $s(\cdot)$ are likely to evolve similarly to market shares $p(\cdot)$. The general relation between $p(\cdot)$ and $s(\cdot)$ is subtle, and one cannot infer the inequality effects of $s(\cdot)$ from those of $p(\cdot)$, and vice-versa, without imposing more structure on $\Pi(i)$.

Things tie up if costs and benefits are power functions as in Section 3.2.4. Let

$$
\Pi(i)=p(i)^{\alpha} \hat{g}(i, T ; x)-p(i)^{\beta} \hat{\varphi}(i, T)
$$

where, in general, $\alpha \equiv u(T ; x)$ and $\beta \equiv z(T)$ with $\beta>\alpha>0$, such that the first-order conditions have the form (19), and (20) applies ${ }^{24}$ Because $s(\cdot)$, like $p(\cdot)$, is a density with support $[0,1]$, Corollary 5 implies that $d x$ induces inequality effects on $s(\cdot)$ if and only if the ratio $s(i) / s(j)$ is

\footnotetext{
${ }^{24}$ The requirement that $\beta>\alpha$ follows from strong quasiconcavity.
} 
not constant for some $i, j \in I$. It is easily verified that

$$
\frac{s(i)}{s(j)}=\left(\frac{p(i)}{p(j)}\right)^{\alpha} \frac{\hat{g}(i, T(x) ; x)}{\hat{g}(j, T(x) ; x)}=\left(\left(\frac{\hat{g}(i, T(x) ; x)}{\hat{g}(j, T(x) ; x)}\right)^{\beta}\left(\frac{\hat{\varphi}(j, T(x))}{\hat{\varphi}(i, T(x))}\right)^{\alpha}\right)^{\frac{1}{\beta-\alpha}}
$$

Thus, relative payoff shares (22) display a similar structure as relative market shares (20). If $\alpha, \beta$ are constant, the market and payoff shares essentially follow the same inequality patterns.

Proposition 4 Suppose that $\frac{s(i)}{s(j)}$ is given by 22 , where $\beta>\alpha>0$ are constant. If either $\hat{g}(\cdot)$ or $\hat{\varphi}(\cdot)$ is invariant to the agent index $i$, then

i) $x$ is inequality preserving in $s(\cdot) \Leftrightarrow x$ inequality preserving in $p(\cdot)$,

ii) $d x$ induces a monotonic $O R(I R)$ of $s(\cdot) \Leftrightarrow d x$ induces a monotonic OR (IR) of $p(\cdot)$.

If both ratios on the RHS of (22) are strictly increasing (decreasing) in $x$, then $d x>0$ induces a monotonic OR (IR) of both $p(\cdot)$ and $s(\cdot)$.

By Proposition 4, $s(\cdot)$ and $p(\cdot)$ can display dissimilar patterns only if both ratios on the RHS of 22 depend on $x$, but one ratio increases in $x$ while the other decreases. In terms of our later applications, we often find that the ratio of marginal benefits, $\hat{g}(i) / \hat{g}(j)$, is independent of $x$, implying that $p(\cdot)$ and $s(\cdot)$ ought to display the same inequality pattern.

Although $p(\cdot)$ and $s(\cdot)$ may behave similarly, this does not generally imply that these distributions coincide. However, as (22) suggest, the relation between the two can be disentangled if payoffs are of the form (21).

Lemma 3 The market and payoff share functions coincide $(s(i)=p(i) \forall i)$ if and only if $\frac{s(i)}{s(j)}=\frac{p(i)}{p(j)} \forall i, j$. If $\frac{s(i)}{s(j)}>(<) \frac{p(i)}{p(j)} \forall j \triangleright i$, then $s(\cdot)$ is a monotonic OR (IR) of $p(\cdot)$.

To avoid misunderstanding, the statement that " $s(\cdot)$ is an OR of $p(\cdot)$ " means that the two densities $s(\cdot)$ and $p(\cdot)$ are ranked by the OR criterion analogously to Definition 7 . That is, $s(\cdot)$ crosses $p(\cdot)$ from above. Note that Lemma 3 can be used to rank any two decreasing densities with identical equivalence classes and support according to the OR-criterion.

\subsection{Comparative-Statics of $T(x)$}

By (10), sign $T^{\prime}(x)$ matters for $R$, and can affect the rotational pattern. We now present a simple procedure to determine $\operatorname{sign} T^{\prime}(x)$, exploiting the transformation of the model into a competition for market shares. The procedure works as follows.

- Step I: Fix an arbitrary agent $i$, and suppose that $x$ and $T$ are exogenous parameters. Then, equation (5) implicitly determines a function $p(i ; T, x)$. Use the Implicit Function Theorem to determine the partial derivatives $p_{x}(i ; T, x)$ and $p_{T}(i ; T, x)$.

- Step II: Define $G(T, x) \equiv \int p(i ; T, x) d i$. Use Step I to determine $G_{T}(T, x)$ and $G_{x}(T, x)$. Use the equilibrium equation $G(T, x)=1$ to determine $T^{\prime}(x)$. 
We illustrate this procedure by proving the following result.

Lemma 4 Let Assumptions 1 1 be satisfied, $g_{x}(i)>0$ and $\varphi_{x}(i)=0 \forall i$. Then $T^{\prime}(x)>0$.

Proof: Consider an arbitrary agent $i$. Step I: By (A1), equation (5) must have a unique solution $p(i ; T, x)$ for any given $T, x$. Quasiconcavity (A1) and $g_{x}>0$ further imply that $p_{x}(i ; T, x)>0$. Likewise, quasiconcavity and the fact that $g_{T}(i)<\varphi_{T}(i)$ by (A2), together assure that $p_{T}(i ; T, x)<0$. Step II: For $G(T, x) \equiv \int p(i ; T, x) d i$ it follows from Step I that $G_{x}(T, x)>0$ and $G_{T}(T, x)<0$. Based on the equilibrium equation $G(T, x)=1$, the Implicit Function Theorem and Step II implies that $T^{\prime}(x)>0$.

In the literature on aggregative games the comparative-statics of aggregate quantities is a prominent questions. This literature has found creative ways to establish monotone comparativestatics of aggregate quantities (see, e.g., Corchon, 1994, Cornes and Hartley, 2012; Acemoglu and Jensen, 2010, 2013, Camacho et al., 2018). We complement this particular aspect by offering a simple procedure to determine $\operatorname{sign} T^{\prime}(x)$ by exploiting the equivalent representation of the model as a competition for market shares. Our main contribution, however, is to exploit the aggregative structure to study the array of inequality effects across all agents.

More generally, one should note that any monotonicity of the action variable in the parameters, as commonly studied by the literature on monotone comparative statics, is of little relevance to our inequality analysis. The mere fact that, e.g., actions or payoffs are increasing in $x$ does not generally pin down the inequality effects.25

\section{Applications}

We now analyze the inequality effects in a variety of examples, thereby pursuing a two-folded goal. First, we want to facilitate the understanding and demonstrate the applicability of our inequality results by putting them to work in various competition models ${ }^{26}$ Second, we seek to extend the set of testable predictions regarding market inequality within the competition models we consider.

Procedural Remark We study the inequality effects in the various applications by essentially following the analytical procedure outlined in Section 3. That is: We first rewrite the payoff function, such that a competition for market share results. We then assert whether the market condition $x$ is a level variable. If yes, this may allow us to directly use some of our general inequality results about level variables. If not, we proceed by deriving the sign of the directaggregative effect $R$ based on (5) which, by Theorems 1 and 2 , allows us to determine whether an inequality effect arises and, if yes, whether it adopts the pattern of a rotation. Alternatively, we can try to use Theorem 3 or Theorem 4 to detect monotonic rotations, which is particularly helpful if payoffs involve power functions.

\footnotetext{
${ }^{25}$ Our approach does not depend on any monotonic relations between parameters and equilibrium actions.

${ }^{26}$ In Appendix B.8 we also study an extensive application to international trade in a discrete choice setting. Also see Hefti and Lareida (2020) for a stand-alone application where we extend the framework to allow for agent entry.
} 


\subsection{Inequality Effects in Monopolistic Competition}

Our first application studies firm-side market inequality in models of monopolistic competition with CES-demand (Dixit and Stiglitz, 1977). This type of competition plays a central role, e.g., in international trade or IO. Specifically, we study how changes in total disposable consumer income, the industry-level of productive efficiency or the intensity of competition as captured by the consumers' "love-of-variety" influence the equilibrium distribution of firm-side market shares and payoffs under a variety of assumptions on the production functions.

Some of our results connect to international trade. It is well-known that firm heterogeneity is indispensable for understanding many empirical regularities in trade data related to firm-side market inequality (Redding, 2011; Gervais, 2015). However, the question how the firm market or payoff shares depend on common market conditions, such as an increasing consumer income due to international integration (Mrázová and Neary, 2017), has generally received little attention. In this respect, our framework allows for a level of generality in the inequality analysis that goes beyond the standard case of constant marginal costs on the producer side.

\subsubsection{The Model}

There is a continuum of consumers, indexed by $\iota \in[0,1]$, each endowed with CES utility

$$
U(\iota)=\int_{0}^{1} r_{s} q_{s}(\iota)^{\sigma} d s, \quad \iota \in[0,1]
$$

where $s \in[0,1]$ denotes a differentiated product supplied by a single firm ${ }^{27}$ Further, $r_{s}>0$ measures the importance of product $s$ to consumers (e.g., the quality), $q_{s}(\iota) \geq 0$ is the respective quantity demanded by $\iota$, and $\sigma \in(0,1)$ the elasticity of substitution. Consumer $\iota$ has disposable income $I(\iota)>0$, and chooses each $q_{s}(\iota)$ to maximize (23), subject to the budget constraint $\int P_{s} q_{s}(\iota) d s=I(\iota)$, where $P_{s}>0$ is the price of product $s$. For $\eta \equiv \frac{1}{1-\sigma}>1$, this optimization problem leads to an aggregate consumer demand for product $i \in[0,1]$ given by

$$
q_{i}=\frac{I r_{i}^{\eta} P_{i}^{-\eta}}{\int r_{s}^{\eta} P_{s}^{1-\eta} d s}, \quad I \equiv \int I(\iota) d \iota>0 .
$$

Firm $i \in[0,1]$ produces its quantity $q_{i}$ according to $q_{i}=\frac{\theta}{c_{i}} f_{i}\left(x_{1}, \ldots, x_{K}\right)$, where $\theta>0$ is a common and $c_{i}>0$ and individual productivity parameter. We assume that the production function is homogeneous of degree $1 / \gamma_{i}, \gamma_{i} \geq 1$, where the production factors $x_{1}, \ldots, x_{K}$ are acquired in competitive factor markets. Thus, each firm has a cost function of the form $\Phi\left(i, q_{i}\right)=$ $\frac{c_{i}}{\theta} q_{i}^{\gamma_{i}}$. This subsumes the case of constant marginal costs (where $\gamma_{i}=1 \forall i$ ), which is the most common assumption in applications of monopolistic competition, in particular in international trade (Melitz, 2003). Our analysis is not confined to linear costs, nor do we generally require that all production functions be homogeneous of the same degree.

We note that firms can differ ex ante in various ways. First, the firms can differ in their productive efficiency $c_{i}$ (a larger $c_{i}$ means a lower productivity) or in their qualities $r_{i}$. Second, firms can differ in their returns to scale $\gamma_{i}$. As our inequality analysis below shows, it plays a

\footnotetext{
${ }^{27}$ It is convenient to indicate firms by using subscripts, i.e., we write $r_{s}$ instead of $r(s)$.
} 
decisive role, whether there is ex ante heterogeneity in the former two or in the latter.

\subsubsection{Inequality Analysis}

Because we allow for non-linear costs, it is hard to study the inequality effects with the conventional approach, in particular also as we allow for an arbitrary number of firm types. To make use of our inequality tools, the first step is to restate monopolistic competition as a competition for market shares ${ }^{28}$ Let the market share $p_{i}$ be the fraction of consumption expenditures earned by firm $i$,

$$
p_{i} \equiv \frac{P_{i} q_{i}}{\int P_{s} q_{s} d s}
$$

where $q_{i}$ is given by 24 for each price $P_{i}$. In terms of our framework, the price $P_{i}$ thus corresponds to the "action variable" $t_{i}$. For any given price profile $P$ we define the aggregator function $Z(P)=\int r_{s}^{\eta} P_{s}^{1-\eta} d s$, where the value $T=Z(P)$ can be interpreted as a preferenceweighted inverse price index ${ }^{29}$ These definitions and (24) yield the payoff function

$$
\Pi_{i}=P_{i} q_{i}-c_{i} q_{i}^{\gamma_{i}}=I p_{i}-w_{i} I^{\gamma_{i}} p_{i}^{\frac{\gamma_{i} \eta}{\eta-1}} T^{\frac{\gamma_{i}}{\eta-1}}, \quad w_{i} \equiv \frac{c_{i}}{\theta} r_{i}^{-\frac{\gamma_{i} \eta}{\eta-1}},
$$

which is of type (4). To see that $\left(\left\{\Pi_{i}\right\}, Z(\cdot)\right)$ indeed constitute a competition for market shares, note that $p_{i}$ can be equivalently stated as $p_{i}=\frac{r_{i}^{\eta} P_{i}^{1-\eta}}{T}$, which shows that $(3)$ is verified. Thus, we can apply the tools from Section (24) to study the inequality effects.

Each firm chooses its market share $p_{i}$ to maximize (26) and, after some algebraic manipulation, the optimality condition (5) evaluates to

$$
g(i) \equiv\left(\frac{\eta-1}{\eta \gamma_{i}}\right)^{\eta-1} I^{\left(1-\gamma_{i}\right)(\eta-1)} w_{i}^{1-\eta}=p_{i}{ }^{\eta\left(\gamma_{i}-1\right)+1} T^{\gamma_{i}} \equiv \varphi(i),
$$

in this model 30

The market conditions of interest are disposable income $I$, consumer preferences $\eta$, productive efficiency $\theta$ or the quality level $r$ (assuming that $r_{i}=r \forall i$ ). Considering (27), we observe that the equilibrium functions $g(\cdot), \varphi(\cdot)$ display the Power Function property from Proposition 3 if and only if $\gamma_{i}=\gamma \forall i \in I$, i.e., if and only if all firms are subject to exactly the same returns to scale. Moreover, disposable income $I$ and quality $r\left(r_{i}=r \forall i\right)$ are level variables if and only if all firms are subject to the same returns to scale, which is easy to see by applying the separability criterion from Section 3.1.1 to $g(i)$ in (27). This suggests that the homogeneity degree $\gamma_{i}$ plays a central role for the inequality effect, and we therefore consider separably the cases where i) the scale effects are identical, and ii) the scale effects are heterogeneous.

Identical Scale Effects We begin with the case where $\gamma_{i}=\gamma \geq 1 \forall i \in I$. The firms can be ex ante heterogeneous in $c_{i}$ or $r_{i}$, where we assume the heterogeneity to be such that $w_{i}$ is

\footnotetext{
${ }^{28}$ Equilibrium existence and uniqueness for this model are well-known; both also follow from our general Existence Theorem B.2 in Appendix B.2

${ }^{29}$ This is an example where the aggregator function $Z(\cdot)$ does not equal the sum of actions (i.e., prices).

${ }^{30}$ Note that $g(i)$ and $\varphi(i)$ as we defined them in (27) do not exactly coincide with marginal benefits and costs, which is not important, however, from the perspective of our inequality tools.
} 
increasing over agent types, such that $p_{i}$ is a Class I or Class II density ${ }^{31}$ If all firms have the same returns to scale, then $I, \theta$ and a common quality level $r$ (for $r_{i}=r \forall i$ ) must be level variables. Corollary 1 then immediately implies that these variables must be inequality preserving. Consumer preferences $\eta$, as well as the reruns to scale $\gamma$, are not level variables but common market conditions, as all firms in the market are affected by them, although differentially. We note from Proposition 3 any rotation must be monotonic if the direct-aggregative effect $R$ is sign-uniform for all firms given that (27) verifies the power function property. Moreover, the power function structure allows us to invoke Lemma 3 to infer how the payoff shares evolve. The following proposition summarize the inequality effects of this model.

Proposition 5 Suppose that all firms have identical returns to scale $\left(\gamma_{i}=\gamma \forall i\right)$ and $w_{i}$ is increasing over firm types. The inequality effects of consumer-side parameters $\eta, I$, and technologyside parameters $\gamma, \theta$ as well as $r$ (if $r_{i}=r \forall i$ ) are summarized by Table 1, where all rotations are monotonic. Further, the market and payoff shares coincide $\left(p_{i}=s_{i} \forall i\right)$.

\begin{tabular}{|l|c|c|c|c|c|c|}
\hline & $p(\cdot)$ & $s(\cdot)$ & $\frac{p_{i}}{p_{j}}$ & $\frac{\Pi_{i}}{\Pi_{j}}$ & $\frac{P_{i}}{P_{j}}$ & $\int \Pi_{i} d i$ \\
\hline$d \eta>0$ & OR & OR & $\uparrow$ & $\uparrow$ & $\gamma>1: \operatorname{sign}\left(\frac{r_{i}}{r_{j}}-\frac{c_{i}}{c_{j}}\right)$ & $\downarrow$ \\
\hline$d I>0$ & - & - & - & - & - & $\uparrow$ \\
\hline$d \gamma>0$ & IR & IR & $\downarrow$ & $\downarrow$ & $\gamma>1: \operatorname{sign}\left(\frac{r_{i}}{r_{j}}-\frac{c_{i}}{c_{j}}\right)$ & $\uparrow$ \\
\hline$d \theta, d r>0$ & - & - & - & - & - & - \\
\hline
\end{tabular}

Table 1: Inequality effects with identical returns to scale

By Proposition 5, income $I$ as well as industry-wide quality and efficiency levels $r, \theta$ are inequality-preserving under homogeneous scale effects, both with respect to market and payoff shares. Observing that changes in $\theta$ could also be interpreted as introducing or altering a production tax (or subsidy), Proposition 5 implies that such policy measures are inequality preserving as well.

Note that an increase in income, however, is not entirely neutral. In particular, $d I>0$ induces a rich-gets-richer effect in terms of absolute profits $\Pi_{i}$. That is, the gap $\Pi_{i}-\Pi_{j}$ is strictly increasing in $I$ for any given pair of firms with $j \triangleright i$. In particular, this means that the strongest firms gain most, while market shares remain stable. To see this, note from Table 1 that all payoff shares remain constant while the aggregate payoff increases. These two facts imply that each firm must realize a strictly higher payoff, and Lemma 2 then shows that the payoff gaps must increase.

By contrast, variations in the willingness to substitute $\eta$ or in the returns to scale $\gamma$ induce monotonic rotations of the market and payoff shares. With stronger substitutes $(d \eta>0)$, competition becomes more intense which, intriguingly, lets already rich firms expand their market size, thereby marginalizing small firms. Likewise, market shares are less equally dispersed with stronger returns to scale (lower $\gamma$ ). This implies that the standard case of constant marginal $\operatorname{costs}(\gamma=1)$ induces most market inequality among all technologies with non-increasing returns to scale.

\footnotetext{
${ }^{31}$ For example, this holds if $c_{i}$ is increasing while $r_{i}=r \forall i$.
} 
Finally, it is interesting to observe in Table 1 that relative prices can move quite independently from market shares and relative payoffs if $\eta$ or $\gamma$ changes. For instance, relative prices $\frac{P_{i}}{P_{j}}$ never respond to $\eta$ and $\gamma$ in the special case of constant marginal costs, while they change with non-linear production costs, depending on the quality-cost ratios.

Why are income $I$, efficiency $\theta$ and quality $r$ inequality preserving, while $\sigma$ and $\gamma$ are not? To understand this intuitively, note from (27) that, e.g., and increase in income has a uniform effect on all firms, and thus provides an identical incentive for all firms to increase their market shares. Such an increase, however, is more costly to bear for firms that already possess a large market share due to increasing marginal costs. The multiplicative separable structure of payoffs now implies that these two effects level off in equilibrium, resulting in a stable market share distribution.

By contrast, the common market variables $\gamma$ and $\eta$ exert differential effects on the various firms, leading to effects that are not offset by equilibrium forces. To see why, note that if products are stronger substitutes ( $\eta$ increases), competition is intensified, implying that all firms tend to save costs by cutting back on market shares. In addition, $d \eta>0$ also increases how sensitively marginal costs respond to changes in $p_{i}$, and more so for firms with larger market shares. This allows such firms to adopt better to the more competitive market environment than their smaller counterparts.

We remark that our inequality tools allow for a richer analysis than conducted here. As an illustration, suppose that $r_{i}=r$ while $c_{i}$ is increasing over firm types. Consider an innovation which increases efficiency $\left(d c_{i}<0\right)$, but differentially so for different firms (i.e., $d c_{i} \neq d c_{j}$ ). Such an innovation is inequality preserving if and only if $\frac{d c_{i}}{c_{i}}=\frac{d c_{j}}{c_{j}}$ for all firms, meaning that the innovation affects each firm in a proportional way (and thus works like a level variable). By contrast, if $\frac{d c_{i}}{c_{i}}<\frac{d c_{j}}{c_{j}}<0 \forall j \triangleright i$, such that the firms with a smaller market share benefit proportionally less from the innovation, one can use Theorem 3 to prove that the innovation triggers a monotonic OR of the market and payoff shares.

Heterogeneous Scale Effects We now consider the formally more challenging case, where firm heterogeneity originates from different returns to scale. Let $r_{i}=c_{i}=1 \forall i \in I$, such that $w_{i}=1 / \theta$ in (27), and $\gamma_{i} \geq 1$ be an increasing and step function. We assume parameters to be such that each firm produces $q_{i} \geq 1 \forall i \in I$ in equilibrium, which assures that $p_{i}>p_{j}$ for $j \triangleright i$, such that $p_{i}$ belongs to Class I, and $P_{i}<P_{j}$ as well as $q_{i}>q_{j}$ for any $j \triangleright i$ 32

Proposition 5 has shown that the common efficiency level $\theta$ or income $I$ are inequality preserving with identical returns to scale. The next two propositions highlight that this conclusion breaks down once firms differ in their returns to scale.

Proposition 6 A common increase in efficiency $(d \theta>0)$ induces a monotonic OR of market and payoff shares. Winners $\left(d \Pi_{i}>0\right)$ and losers $\left(d \Pi_{i}<0\right)$ always coexist, where a firm wins if and only if its respective market share $p_{i}$ increases. Further, the ratios $\frac{\Pi_{i}}{\Pi_{j}}, \frac{P_{j}}{P_{i}}$ and $\frac{q_{i}}{q_{j}}$ increase whenever $j \triangleright i$, and the industry payoff $\Pi(\theta) \equiv \int_{I} \Pi_{i}(\theta)$ di verifies $\Pi^{\prime}(\theta)>0$.

\footnotetext{
${ }^{32} q_{i} \geq 1$ holds if $I$ is large (or $c$ small) enough.
} 
The proof essentially consists of algebraic manipulations in order to apply Theorem 3 .

Proposition 6 considers the case of an increase in efficiency (or a reduction in a production tax), but draws a substantially different picture than its counterpart with identical scale effects. With heterogeneous scale effects, $d \theta>0$ increases market inequality, creates winners and losers in terms of absolute payoffs, decreases the aggregate payoff and affects relative prices, while all these effects did not arise previously.

The reason for these deviations are the differential returns to scale. In particular, while $\theta$ still is a level variable, the multiplicative separability of $\varphi(i)$ in $(i, p)$ and $T$ is violated, meaning that $\theta$ cannot be inequality preserving anymore by Corollary 1 . Intuitively, the increase in $\theta$ unambiguously decreases all individual prices $P_{i}$, which essentially is a standard passthrough effect reflecting lower marginal costs. However, the leading firms manage to garner a disproportionally larger amount of consumer demand as they can afford to decrease their prices disproportionally more due to their advantages in the returns to scale, which results in a larger market share and payoff as before.

One notable consequence is that if the firm heterogeneity is rooted in different returns to scale, then the leading firms have an incentive to innovate if innovation increases $\theta$, even though the innovation spills over to all competitors. By contrast, laggards are hurt by such innovations, and therefore do not seek them.

Turning to income effects, the following proposition shows that consumer income seizes to be inequality preserving once the firms differ in their returns to scale. Specifically, an increasing income now implies that quantity growth coexists with a growing inequality of market shares and payoffs.

Proposition 7 An increase in consumer income $d I>0$ triggers a monotonic OR of market and payoff shares, increases all quantities $q_{i}$ and also increases relative quantities $\frac{q_{i}}{q_{j}}$, relative profits $\frac{\Pi_{i}}{\Pi_{j}}$, relative market shares $\frac{p_{i}}{p_{j}}$ and relative prices $\frac{P_{j}}{P_{i}}$ for any $j \triangleright i$.

The reason is that now $I$ fails to be a level variable, meaning that $I$ has a differential impact on the firms, which jointly with the differential effect on marginal costs as in the case of $d \theta>0$, generates an inequality effect favoring the firms with larger market sizes.

\subsubsection{Discussion}

Propositions 5- 7 reveal substantial differences depending on whether all firms have the same scale effects or not. As the case of identical returns contains the standard premise of constant marginal costs, our finding complements and generalizes a result by Mrázová and Neary (2017). These authors study how the shape of demand, summarized by its "demand manifold", determines the pass-through and competition effects with constant marginal cost firms. They show that consumer income does not affect the dispersion of firm payoffs in case of CES demand. Proposition 5 extends this result to non-linear costs, showing that the distributional neutrality of income with CES demand is actually driven by the premise of homogeneous scale effects across firms. 
Proposition 7 complements their finding by showing that level variables seize to be inequality preserving once firms differ in the economies of scale, despite CES-demand. Specifically, income growth, common efficiency gains or general cost reductions lead to quantity growth jointly with increasing relative prices, and a growing inequality in market and payoff shares. More generally, level variables now induce inequality effects, because the firms with comparably favorable scale economies can respond more flexibly to a common change of the economic environment.

It is worthwhile to mention that these inequality effects are not driven by the firm-side market power contained in monopolistic competition; in Appendix B.6 we prove that the same inequality effects arise with perfectly competitive firms 33 Moreover, we shall see in the next sections that these inequality effects are a vindication of a more general pattern, which occurs in symmetric competitions, where the equilibrium value per unit of market share is identical to all firms.

\subsection{Market Inequality in Models of Perfect Competition}

This section derives the inequality effects for various examples of perfect competition, considering inequality on the firm- and consumer-side. For consumers, our notion of market inequality concentrates on consumption inequality given its importance for welfare Attanasio and Pistaferri, 2016).

\subsubsection{Perfect Competition}

We first consider the firm-side inequality effects in a perfectly competitive market with exogenous demand. The insights obtained from this setting are helpful for situating the previous findings from monopolistic competition, and useful also for the general equilibrium applications we study in Section 4.2 .2 .

Let $i \in[0,1]$ denote a perfectly competitive firm in a market for a homogeneous good. Each firm takes the market price $P$ as given and supplies a quantity $q_{i} \geq 0$, produced with a strictly convex cost function $\Phi(i, \cdot)$, and $\Phi(i, 0)=0$. As in Section 4.1, we think of $\Phi(i, q)$ as arising from a production function, where the inputs are acquired on competitive factor markets. As before, this implies that $\Phi(i, q)=c_{i} q^{\gamma_{i}}$ if and only if the production function is $1 / \gamma_{i}$-homogeneous. The inverse demand is $P=P\left(\int q_{i} d i ; x\right)$, where $x$ denotes a demand shifter with $P_{x}>0$.

As in Section 4.1. the market share $p_{i} \equiv \frac{P q_{i}}{\int P q_{s} d s}$ is the share of consumption expenditures earned by firm $i$. Further, $T \equiv \int q_{i} d i$ is total market supply, where $P_{T}(T ; x)<0$ by the Law of Demand. Because $p_{i}=q_{i} / T$, condition (3) is verified, and restating the payoff as a competition for market shares yields

$$
\Pi_{i}=P q_{i}-\Phi\left(i, q_{i}\right)=p_{i} P T-\Phi\left(i, p_{i} T\right)
$$

Thus, the equilibrium equation (5) takes on the form

$$
P(T ; x)=\varphi\left(i, p_{i} T\right)
$$

\footnotetext{
${ }^{33}$ Actually, we even find that market power per se does not induce a different dispersion of market shares: While market power increases the level of profits, equilibrium forces balance in a way that the market share distribution of perfect competition and monopolistic competition must perfectly coincide.
} 
Regarding the ex ante heterogeneity, we assume that there are finitely many cost types, such that $p(\cdot)$ is Class I, where $\Phi(i, q)<\Phi(j, q)$ and $\varphi(i, q)<\varphi(j, q)$ whenever $j \triangleright i$ and $q>0$. This assures that $p_{i}>p_{j}, \Pi_{i}>\Pi_{j}$ for $j \triangleright i$ in equilibrium.

The equilibrium equation (29) is a variant of (11), and $x$ is a level variable. Thus, by Corollary 1. the inequality effects depend entirely on the cost functions $\varphi(i)$, i.e., the slope or curvature of market demand $P(T ; x)$ plays no role for whether inequality effects arise.

It is easy to check that $T^{\prime}(x)>0$, e.g., by using the procedure outlined in Section 3.3. Because $x$ is a level variable and $q_{i}=p_{i} / T$, Corollary 2 then tells us that $x$ is inequality preserving if and only if all firms have exactly the same returns to scale, i.e., all cost functions are of the form $\Phi(i, q)=c(i) q^{\gamma}$. If the latter is violated, 4 tells us that the elasticity of marginal costs, $\psi_{i}=\frac{\varphi_{q}\left(i, q_{i}\right) q_{i}}{\varphi_{q}\left(i, q_{i}\right)}$, determines whether a rotation occurs - and if yes, these rotations must even be partially monotonic by Corollary 6 . These insights are summarized in the following proposition.

Proposition 8 The demand shifter $x$ is inequality preserving if and only if $\Phi(i, q)=c_{i} q^{\gamma}$, $\gamma>1, \forall i \in I$. Further, $d x>0$ increases the equilibrium price $P=P(T ; x)$ and the quantity $q_{i}$ supplied by each firm, and induces a partially monotonic OR (IR) of the market shares in the sense of (17) (in the sense of (18)) if $\psi_{j}>(<) \psi_{i} \forall j \triangleright i$ in equilibrium.

The elasticity $\psi_{i}$ captures how easily a firm can adjust its production to changing market conditions, which is crucial for whether a firm manages to expand its market share following an upward shift in market demand. If $\Phi(i, q)=c_{i} q_{i}^{\gamma} \forall i$, then all firms are equally able to respond to the demand increase, resulting in stable market shares. However, if the firms with larger market shares also are those that can adjust more easily, i.e., $\psi_{i}<\psi_{j}, j \triangleright i$, this leads to a redistribution of market shares from the tail to the top. The equilibrium adjustments are even such that the firms who gain market shares can increase these more than proportionally compared to any other firm with a smaller market share. These findings are reversed if $\psi_{j}<\psi_{i}$ $\forall j \triangleright i$, meaning that the firms with the smallest market shares are those to adjust most flexibly.

Market and Payoff Shares The simple structure of (29) allows us to say more about the relation between market shares $p(\cdot)$ and payoff shares $s(\cdot)$, mostly due to Lemma 3 .

Proposition 9 Market and payoff shares perfectly coincide $\left(s_{i}=p_{i} \forall i \in I\right)$ if and only if $\Phi(i, q)=c_{i} q^{\gamma}, \forall i \in I$, and $s(\cdot)$ is a monotonic OR (IR) of $p(\cdot)$ if and only if $\psi_{j}<(>) \psi_{i} \forall j \triangleright i$. Further, $x$ increases all absolute payoffs $\Pi_{i}$ and payoff gaps $\Pi_{i}-\Pi_{j}, j \triangleright i$.

In general, the elasticity $\psi_{i}$ depends on the agent index $i$ as well as on the equilibrium quantity $q_{i}$. The two extreme cases, where $\psi_{i}$ depends either only on $i$ or only on $q_{i}$ allow for stronger results.

First, $\psi_{i}$ depends on $i$, but not on $q, \forall i$, if and only if $\Phi(i, q)=c_{i} q^{\gamma_{i}}$, which corresponds to the case where the firms differ in their returns to scale, as in Section 4.1. If, e.g., $\Phi(i, q)=c q^{\gamma_{i}}$, and $\gamma_{i}<\gamma_{j}, j \triangleright i$, then $s(\cdot)$ must be a monotonic IR of $p(\cdot)$ by Proposition 9, and an increase in demand must induce a monotonic OR of $p(\cdot)$ and $s(\cdot)$ by Corollary 7 and Proposition 4. 
Second, the case where $\psi(i)$ depends only on the quantity $q_{i}$ but not directly on the firm index $i$ occurs if and only if the costs are of the form $\Phi(i, q)=c_{i} H(q)$. In this case, it is easy to verify that $d x>0$ induces an OR (IR) of $p(\cdot)$, and $s(\cdot)$ is IR $(\mathrm{OR})$ of $p(\cdot)$, if $\frac{H^{\prime \prime}(q) q}{H^{\prime}(q)}$ is strictly decreasing (increasing) in $q>034$

Proposition 9 predicts a tight relation between market and payoff shares and the inequality effects induced by $d x>0$. One particular conclusion, in terms of observables, is that $x$ must induce inequality effects if and only if the market and payoff share distributions disagree.

Corollary 8 The market and payoff shares coincide if and only if $x$ is inequality preserving. If $p(\cdot)$ is a monotonic OR (IR) of $s(\cdot)$, then $d x>0$ must induce an $O R(I R)$ of $p(\cdot)$.

By Corollary 8 not every pattern can arise. For example, we cannot have that $p(\cdot)$ and $s(\cdot)$ are ranked in terms of a monotonic OR while an upward shift in demand decreases the inequality in the market share distribution.

Discussion Compared to monopolistic competition, the above model is adequate for situations where the market is small relative to the economy, such that wealth or spillover effects between markets are negligible. Nevertheless, both models yield similar inequality effects with respect to a level variable $x: x$ is inequality-preserving if and only if all firms operate with identical returns to scale. By contrast, an increase in $x$ generates an increasing polarization of the market shares if firms differ in their returns to scale. The underlying reason is that both models feature a symmetric competition, in thus that the value per unit of market share is identical for all firms (see Section 4.4).

Another interpretation of $d x>0$ in the current model is that this amounts to an introduction or a decrease in a quantity or sales tax - the former yields $P(T ; x)=P(T)-x$ and the latter $P(T ; x)=(1-x) P(T)$. Such a tax is a level variable, meaning that it always is inequality preserving if and only if all firms feature exactly the same returns to scale. By contrast, if the firms with the largest market shares also have the lowest elasticity of marginal costs, e.g., because of favorable returns to scale, then a tax induces an IR of market shares, while a subsidy creates an OR of $p(\cdot)$.

\subsubsection{General Equilibrium Applications}

Partial Equilibrium In this application, we consider the inequality effects of a common market condition, such as a market-level innovation or a tax, in a partial equilibrium setting. This setting allows us to study the interdependence between the inequality effects caused on either market side if there are no wealth effects on the consumer side. By contrast to most of the existing work on taxation, which focuses on the tax incidence between firms and consumers (see, e.g., Mieszkowski, 1969), our approach elicits the inequality effects within each market side.

\footnotetext{
${ }^{34}$ The case where $H(q)=q^{\gamma}$ exactly yields the intermediate case, where $\psi_{i}$ is constant and equal across all agents, making $x$ inequality preserving.
} 
Firms and consumers are indexed by $i \in[0,1]$ and $i_{c} \in[0,1]$, respectively. Utility is quasilinear $U\left(i_{c}\right)=m_{i_{c}}+V\left(i_{c}, q_{i_{c}}^{d}\right)$, where $m_{i_{c}} \in \mathbb{R}$ is a numeraire, and $q_{i_{c}}^{d} \geq 0$ is the demand for the consumption good in the market under study. Each consumer is endowed with $\omega_{i_{c}}>0$ of the numeraire, and a share $s_{i_{c}}$ of total firm profits, where $\omega=\int \omega_{i_{c}} d i_{c}$ and $\int s_{i_{c}} d i_{c}=135$

Consumers are ex-ante heterogeneous in the desirability $V\left(i_{c}, \cdot\right)$ of the consumption good. We assume that $V\left(i_{c}, \cdot\right)$ is strictly increasing and concave, where $v\left(i_{c}, q\right) \equiv \partial_{q} V\left(i_{c}, q\right)$ satisfies $v\left(j_{c}, q\right)<v\left(i_{c}, q\right) \forall q>0$ and any $j_{c} \triangleright i_{c}$, such that consumers are ordered left-to right in terms of preferences. We consider consumption inequality (Attanasio and Pistaferri, 2016) in the consumption good as our notion of market inequality for consumers, and accordingly let $p_{i_{c}}^{d} \equiv q_{i_{c}}^{d} / T^{d}$ denote the share of total consumption $T^{d} \equiv \int q_{i_{c}} d i_{c}$ acquired by consumer $i_{c}$.

Each firm obtains the numeraire on a competitive market, and uses it to produce the consumption good, which is competitively sold to consumers at a price $P$. Then, payoffs have the form (28), where $p_{i}$ is firm $i$ 's share of total consumption expenditures and $T=\int q_{i} d i$.

A competitive equilibrium consists of two market share functions $p_{i}, p_{i_{c}}^{d}>0$, two quantities $T, T^{d}$ such that $T=T^{d}$, and

$$
\begin{array}{lr}
P=v\left(i_{c}, p_{i_{c}}^{d} T\right) & i_{c} \in[0,1] \\
P=\varphi\left(i, p_{i} T\right) & i \in[0,1],
\end{array}
$$

and (30) are the optimality conditions for consumers and firms. Regarding inequality effects, (30) shows that an unilateral change on one market side affects the market share dispersion on the other side only via the direct-aggregative effect ${ }^{36}$ Thus, the inequality effects within the two market sides are generally independent of each other. We now study the inequality effects of a subsidy $\tau>0$ on the supply side, such that the LHS of the 2nd equation in $(30)$ is $P+\tau$.

Proposition 10 An increase in a subsidy, $d \tau>0$, implies that $P^{\prime}(\tau)<0, T^{\prime}(\tau)>0$, and induces the following inequality effects:

- Firms: Propositions 89 and Corollary 8 apply.

- Consumers: $\tau$ is inequality-preserving with respect to consumption shares $p_{i_{c}}^{d}$ if and only if $V\left(i_{c}, q\right)=\alpha_{i_{c}} q^{\mu} \forall i_{c}$. If the q-elasticities of $v\left(i_{c}, q\right)$ are increasing (decreasing) in $i_{c}$ over consumer types, then $d \tau>0$ causes an $O R(I R)$ of $p_{i_{c}}^{d}$.

To understand Proposition 10, the key observation is that $\tau$ is a level variable for each market side through its effects on $P$. Proposition 10 shows that the firm-side and consumer-side inequality effects are determined by the same fundamental logic, while there is no interdependence between the inequality effects. The reason is that wealth effects play no role, and the market price $P$ alone determines the behavior on each market side.

In case of consumers, Proposition 10 implies that if the heavy users are also the consumers with a comparably more elastic marginal utility in absolute value, then the price reduction due to the subsidy leads to more consumption inequality in equilibrium 37

\footnotetext{
${ }^{35}$ As we allow $m_{i_{c}} \in \mathbb{R}$, only interior solutions to the consumer problem can exist, meaning that income inequality plays no role for consumption inequality in the consumption good.

${ }^{36}$ E.g., for consumers, $(30)$ implies that $v\left(i_{c}, p_{i_{c}}^{d} T\right)=v\left(j_{c}, p_{j_{c}}^{d} T\right)$, which shows that any exogenous change on the firm side affects inequality on the consumer side only via $T$.

${ }^{37}$ This occurs, e.g., in case of log-utility $V\left(i_{c}, q\right)=a_{i_{c}} \operatorname{Ln}(1+q)$.
} 
Note that if $\tau$ is a tax rather than a subsidy, Proposition 10 applies analogously with a "reversed sign" of the inequality effects. Further, it is easy to verify that the tax incidence is independent from the inequality effects induced within each market side: Proposition 10 also applies if the subsidy (or the tax) is levied on the consumer side.

Windfall Gains and Income Inequality In this application, we ask how a windfall gain accruing to all consumers affect the inequality on the firm- and consumer-side in a stylized general equilibrium model, given that consumers are heterogeneous in their endowments and ownership shares. Firms and consumers are indexed as before. Consumers initially own all resources and sell them to firms, which produce a homogeneous consumption good. All markets are competitive. Regarding ex-ante heterogeneity, the firms differ in their cost functions as before, and consumers differ in their endowments.

As previously, the firm-side market shares $p_{i}$ refer to the market shares of total consumption expenditure, and $T=\int q_{i} d i$ is aggregate production. If $P$ denotes the price of the consumption good, payoffs are again of the form (28).

Consumers differ in their income, where we distinguish between two key sources of income inequality: Differences in resource endowments or differences in firm ownership shares. Let $\omega_{i_{c}}>0$ denote the resource endowment of consumer $i_{c}$, and $\omega \equiv \int \omega_{i_{c}} d i_{c}$. Further, capital income earned by $i_{c}$ is $S_{i_{c}}=s_{i_{c}} \Pi$, where $s(\cdot)$ is the ex ante distribution of ownership shares $\left(\int s_{i_{c}} d i_{c}=1\right)$, and $\Pi=\int \Pi_{i} d i$ is aggregate profit. Both $\omega_{i_{c}}$ and $s_{i_{c}}$ are (weakly) decreasing in consumer index $i_{c}$, such that consumers are ordered left-to-right in terms of total income. Each consumer $i_{c}$ spends all her income to acquire $q_{i_{c}}^{d}$ units of the consumption good. Thus, if $T^{d} \equiv \int q_{i_{c}}^{d} d i_{c}$ denotes total demand, consumer $i_{c}$ 's consumption share $p_{i_{c}}^{d} \equiv q_{i_{c}}^{d} / T^{d}$ must be equal to her income share of total consumption expenditures, i.e.,

$$
p_{i_{c}}^{d}=\frac{P q_{i_{c}}^{d}}{P \int q_{i_{c}}^{d} d i_{c}}=\frac{\omega_{i_{c}}+S_{i_{c}}}{P T^{d}}
$$

A competitive equilibrium consists of two market share functions $p(\cdot), p^{d}(\cdot)$, and aggregate quantities with $T=T^{d}=\frac{\omega+\Pi}{P}$. On the firm-side, each $p_{i}$ maximizes (28) given that $T=$ $\frac{\omega+\Pi}{P}$. On the consumer-side, each $p_{i_{c}}^{d}$ verifies (31) for $T^{d}=(\omega+\Pi) / P$. We assume the ex ante heterogeneity to be such that $p(\cdot), p^{d}(\cdot)$ are Class I densities. The following proposition summarizes the inequality effects caused by an unforeseen windfall gain such that $d \omega_{i_{c}}=d \omega>0$ for each consumer.

Proposition 11 A windfall gain $d \omega>0$ increases $T, P$, and $\Pi$. The inequality effects are:

- Firms: Propositions 89 and Corollary 8 apply.

- Consumers: (i) If income inequality results from the resource endowment, i.e., $s_{i_{c}}=1$ $\forall i_{c}$, then $d w>0$ induces a monotonic IR of $p^{d}(\cdot)$ (and total income shares). (ii) If income inequality results from the capital endowments, i.e., $\omega_{i_{c}}=\omega \forall i_{c}$, then $d \omega>0$ induces a monotonic OR (IR) of $p^{d}(\cdot)$ (and total income shares) if $\Pi(\omega)$ is strictly convex (concave).

Proposition 11 shows that the firm-side inequality effects are solely determined by the production technologies; neither the ownership structure nor possibly substantial shifts in consumption play 
a role. This is intuitive because, as in our last application, the consumer-side affects the firms only via the market price $P$, showing that $\omega$ is a level variable for firms (but not for consumers).

On the consumer-side, the inequality effects caused by the windfall gain depend on the precise source of the income inequality. If income inequality originates mostly from the resource endowment, then the windfall gain increases equality. The reason is that the income effect caused by the windfall gain is weaker, in relative terms, for wealthier consumers if differences in capital income are negligible. By contrast, the wealthy can benefit from the gain, relative to the poor, if the income inequality originates mainly from the dispersion of capital holdings. The intuition is that the windfall gain has the same uniform effect on total income for all consumers, while the increase in firm profits causes a differential effect. If aggregate profits are convex in $\omega$, then this profit increases faster than linearly, which benefits wealthier consumers more, resulting in an OR of consumption (and total income) shares.

Labor, Leisure and Inequality our last application of perfect competition addresses the connections between consumption and leisure inequality, which is a relatively fresh idea (Attanasio and Pistaferri, 2016). Specifically, we ask how the dispersion of consumption and leisure across consumers depends on the importance of consumption relative to leisure, as well as on labor productivity.

Each consumer $i_{c} \in[0,1]$ owns a unit of perfectly divisible labor. The salary earend in the labor market is used to purchase a quantity $q_{i_{c}}$ of a consumption good. As consumers value leisure, they face a trade-off between leisure and forgone consumption opportunities. We follow most of the literature by assuming that utility is separable in consumption and leisure (see, e.g., Heathcote et al., 2014), i.e.,

$$
u\left(i_{c}\right)=q_{i_{c}}^{\alpha} f_{i_{c}}^{1-\alpha},
$$

where $f_{i_{c}} \in[0,1]$ denotes the amount of leisure, and the parameter $\alpha \in(0,1)$ quantifies the importance of consumption relative to leisure. The budget constraint is $q_{i_{c}}=\left(1-f_{i_{c}}\right) w+S_{i_{c}}$, where $w$ denotes the real wage and $S_{i_{c}}=s_{i_{c}} \Pi \geq 0$ is capital income.

Consumers are ex-ante heterogeneous in their payoff shares, where $s_{i_{c}}$ is decreasing over consumer types. As before, we focus on consumption inequality, where $p_{i_{c}}^{d}=q_{i_{c}} / T^{d}, T^{d}=\int q_{i_{c}} d i_{c}$, denotes $i_{c}$ 's share of total consumption. Assuming an interior solution, constrained maximization of $(32)$ shows that the dispersion of $p_{i_{c}}^{d}$ and leisure $f_{i_{c}}$ are determined by $\alpha\left(w+S_{i_{c}}\right)=p_{i_{c}}^{d} T^{d}$ and $f_{i_{c}}=\frac{\alpha}{1-\alpha} \frac{p_{i_{c}}^{d} T^{d}}{w}$, respectively 38 Thus, we immediately observe that the equilibrium consumption inequality must mirror income inequality, which is a recently rehabilitated empirical fact (Aguiar and Bils, 2015; Attanasio and Pistaferri, 2016).

Each firm hires labor to produce $q_{i} \geq 0$ units of the consumption good, which is produced with labor according to $q_{i}=\left(\frac{\theta}{c_{i}} L_{i}\right)^{1 / \gamma}$, where the efficiency parameter $c_{i}>0$ is increasing over firm types, $\gamma>1$, and $\theta>0$ is labor productivity. Further, $p_{i}$ denotes the share of total consumption expenditure earned by firm $i$, such that the payoff is $\Pi_{i}=p_{i} T-\frac{w c_{i}}{\theta}\left(p_{i} T\right)^{\gamma}$. As the consumer parameter $\alpha$ affects firms via $w, \alpha$ is a level variable. Thus, $\alpha$ and $\theta$ are inequality preserving on the firm-side, which simplifies the analysis of the consumer-side.

\footnotetext{
${ }^{38}$ This interior solution requires that $S_{i_{c}} \leq \frac{\alpha}{1-\alpha} w$ which, for simplicity, we shall assume to hold. The main results carry through if the boundary condition $f_{i_{c}} \leq 1$ becomes binding for some consumers.
} 
We now derive how the importance of consumption relative to leisure affects consumption inequality by deriving the inequality effects induced by an increase in the relative importance of consumption $(d \alpha>0)$. In addition, we also derive the consumer-side inequality effects of an increase in labor productivity $\theta$.

Proposition 12 Let $j_{c} \triangleright i_{c}$. An increase in the importance of consumption (d $\alpha>0$ ) induces a monotonic $O R$ of $p_{i_{c}}^{d}$, and increases relative consumption $\frac{q_{i_{c}}}{q_{i_{c}}}$, relative leisure $\frac{f_{i_{c}}}{f_{j_{c}}}$, aggregate consumption and aggregate labor supply, while wages fall. Moreover, $d q_{i_{c}}>d q_{j_{c}}$ whenever $d q_{i_{c}}>0$. By contrast, labor productivity $(\theta)$ is inequality preserving in terms of market shares, while absolute consumption (and income) gaps increase in $\theta$ (i.e., $d q_{i_{c}}>d q_{j_{c}}$ ).

The intuition for $\alpha$ is that if consumption matters more, consumers seek to supply more labor to afford more consumption. The increased labor supply reduces real wages and increases profits, which benefits capital owners and therefore increases consumption inequality. Because the dispersion of consumption shares, $p_{i_{c}}^{d}$, coincides with the dispersion of leisure, an increase in the propensity to work also increases the inequality in leisure. Additionally, one can show that, as real wages plunge, the poorest may even end up with a lower consumption level despite a higher propensity to consume 39 By contrast, if leisure becomes more important $(d \alpha<0)$, aggregate leisure consumption increases jointly with an increase in relative leisure $f_{j_{c}} / f_{i_{c}}$ of the poor. Such a tendency has been observed, e.g., in US data (Aguiar and Hurst, 2007) 40

By contrast, labor productivity has no inequality effects on the consumer side, independent of the value of $\alpha$, because equilibrium wages and aggregate firm profits increase at the same rate in $\theta$, meaning that the individual consumer income also increases at the same rate. Because consumption and income levels increase, this necessarily implies that the absolute consumption and income gaps must increase between consumers. However, other than in case of $d \alpha>0$, this result depends on the fact that there are no inequality effects on the firm side. Otherwise, aggregate profits may evolve differently than wages, in which case inequality effects necessarily must arise 41

As individual consumption amounts to a constant fraction $\alpha$ of total income $V_{i_{c}} \equiv w+S_{i_{c}}$, Proposition 12 shows that the distribution of income evolves like the distribution of consumption if $\theta$ increases. In particular, $d \theta>0$ induces an OR of the income shares $v_{i_{c}} \equiv V_{i_{c}} / \int V_{s_{c}} d s_{c}$. More generally, Proposition 12 shows that economies with a stronger preference for consumption relative to labor, ceteris paribus, may feature more consumption and income inequality. This broad prediction is reconcilable with empirical evidence comparing European countries with the US, as the latter features more income inequality and a weaker preference for leisure ${ }^{42}$

\footnotetext{
${ }^{39}$ For example, if a positive mass of consumers holds no shares at all $\left(s_{i}=0\right)$ this must always be the case.

${ }^{40} \mathrm{~A}$ more ambitious model could allow $\alpha$ to vary across consumers, e.g., because this matters empirically (Heathcote et al. 2014).

${ }^{4 i}$ This is easy to see, noting that relative income, $V_{i_{c}} / V_{j_{c}}$, is non-constant in $\theta$ if and only if aggregate profits develop at a different pace than wages $\left(\Pi^{\prime}(\theta) / \Pi(\theta) \neq w^{\prime}(\theta) / w(\theta)\right.$.

${ }^{42}$ See, e.g., Blanchard (2004) or Maoz (2010) for leisure preferences in the US relative to Europe. Regarding income inequality, see, e.g., Federal Reserve.
} 


\subsection{Competition for Prizes}

Another prominent instance of competition, that yet differs from the market-based models in the last sections, arises in situations where agent interaction can be described as a competition for prize. An example are contests, where different agents $i \in I$ compete in efforts $t_{i} \geq 0$ to seize a single prize of a fixed value $V>0($ Konrad, 2009). While the literature on contests is comprehensive, papers that study inequality in contests are scarce 43 Other applications suggest that the prize value $V(\cdot)$ is agent-specific or depends on aggregate efforts $T=\int t_{i} d i$. For example, litigation expenditures (Posner, 1992), salary negotiations (Amegashie, 1999), or money invested to obtain a monopoly franchise (Chung, 1996) can influence the revenue earned by the winning agent.

The Model Each agent chooses an effort $t_{i} \geq 0$ that influences her chance of winning a prize. For given efforts and $T \equiv \int t_{i} d i$, the winning chances are determined by $p_{i}=p\left(t_{i}, T\right)$, where $\int p\left(t_{i}, \int t_{s} d s\right) d i=1$, such that $p_{i}$ conceptually is a market share. The success function $p\left(t_{i}, T\right)$ is strictly increasing in $t_{i}$, and zero-homogeneous in $\left(t_{i}, T\right)$. The latter means that only relative efforts matters, which marks a distinct feature of contests relative to market competition, where generally also the absolute values of prices or quantities matter. These assumptions imply that, wlog, we can assume that $p\left(t_{i}, T\right)=t_{i} / T{ }^{44}$ Finally, the prize value generally is determined by a prize function $V\left(i, t_{i}, T\right)$.

Given a cost function $\Phi\left(i, t_{i}\right)$, this yields a competition for market shares with payoffs

$$
\Pi_{i}=p_{i} V\left(i, p_{i} T, T ; x\right)-\Phi\left(i, p_{i} T\right)
$$

where $x$ is a prize shifter $\left(\frac{\partial V(\cdot)}{\partial x}>0\right)$. Consistent with our previous definition, we let $s_{i} \equiv$ $\Pi_{i} / \int \Pi_{i} d i$ denote the (expected) payoff share of agent $i$. We take 33 to satisfy Assumption 1 . such that a unique equilibrium exists. Further, we always assume the ex ante agent heterogeneity to be such that $p(\cdot)$ is Class I.

In the equilibrium, the agents take the aggregate effort $T$ as given when choosing their own efforts. In Appendix B.5, we adjust our approach to include the case where the agents fully account for their own effects on the aggregate, thus embedding the notion of Nash equilibrium.

We first state the inequality effects distinguishing between a homogeneous and a heterogeneous prize function. Next, we study the inequality effects induced by dispersing the prize value over two prices. Finally, we consider an effort-dependent prize function inspired by an advertising context, and discuss the inequality effects of an increased effectiveness of advertising.

Homogeneous Prize Value Consider payoff (33) where the prize is determined by a common value $V=V(T ; x)$ with $V_{x}>045$ This gives $V(T ; x)=\varphi\left(i, p_{i} T\right)$ as equilibrium equation,

\footnotetext{
${ }^{43}$ Perhaps most closely related is $\widehat{\text { Acemoglu and Jensen }}$ (2013), who utilize the aggregative structure to obtain comparative-static predictions of the aggregate and extremal efforts, and sometimes of certain individual strategies. However, their paper does not deal with inequality effects.

${ }^{44}$ Zero-homogeneity implies that $p(t, T)=\hat{p}(t / T)$, and the only function $\hat{p}$ that satisfies $\int \hat{p}\left(t_{i} / T\right) d i=1$, $T=\int t_{i} d i$, for any effort profile $t(\cdot)$ must be linear, i.e., $\hat{p}(x)=x($ Hefti and Lareida 2020).

${ }^{45}$ This includes the case where $V=V(x)$ is a fixed prize.
} 
which is a variant of (11). A direct comparison with (29) shows that price competition and the present contest are variants of the same fundamental structure. Although these models capture substantially different types of competition, they predict the same inequality effects:

Proposition 13 The inequality effects on the market and payoff shares induced by $d x>0$ in the contest model correspond to Propositions 89 and Corollary 8.

In our previous competition models, costs of the form $\Phi(i, t)=c_{i} t^{\gamma}$ amounted to the case of homogeneous returns to scale in production. The analogue in the present model is that the parameter $\gamma$ captures how sensitive the success function responds to changes in efforts. Larger values of $\gamma$ represent "noisier" contests, where the agents have less influence on their success chances (Hefti, 2018). A simple application of Theorem 3 shows that an increase in contest noise induces a monotonic OR of $p(\cdot)$ and $s(\cdot)$, similar to Proposition 5 in monopolistic competition.

Heterogeneous Prize Values We now turn to the case where agents are ex ante heterogeneous in their prize valuations. Let $V_{i}=V(i ; x)$ be increasing over agent types. We consider the inequality effects induced if the valuations increase $\left(d V_{i} \equiv V_{i}^{\prime}(x)>0\right)$, but possibly differentially so for the different agent types (always preserving the agent order). The equilibrium condition (5) thus is $V_{i}=\varphi\left(p_{i} T\right)$. We note that the prize shifter $x$ is a level variable if and only if $d V_{i} / V_{i}=d V_{j} / V_{j} \forall i, j$, where the latter condition means that all prize values must vary in proportion.

Proposition 14 If $x$ is a level variable, then the prize shift is inequality preserving if and only if $\Phi\left(t_{i}\right)=c_{i} t_{i}^{\gamma}$. Let $\Phi\left(t_{i}\right)=$ ct $_{i}^{\gamma}$. Then, a monotonic OR (IR) of market and payoff shares $p(\cdot), s(\cdot)$ results if and only if $\frac{d V(i)}{V(i)} \geq 0$ is strictly decreasing (increasing) in $i$ over all agent types, and payoff shares are a monotonic OR of market shares.

With a power cost function, the payoff shares now must be less equally dispersed than the market shares, other than with a homogeneous prize function. This is due to the fact that agents with large market shares are also the ones to benefit more per unit of market share.

If costs are not given by a power function, the elasticities of marginal costs, $\psi_{i}$, can matter for the inequality effects. A simple combination of 14 and Corollary 4 shows, e.g., that if $\frac{d V_{i}}{V_{i}}>\frac{d V_{j}}{V_{j}}$ and $\psi_{j} \geq \psi_{i} \forall j \triangleright i$, then an OR of $p(\cdot)$ results.

Proposition 14 may help to put our previous results on income inequality in a greater context. The equilibrium equations characterizing the dispersion of consumption (and income) inequality in the models of Propositions 11 and 12 are of the form $V_{i} \equiv \omega_{i}+S_{i}=\left(p_{i} T\right) P$, which shows a formal connection to the present contest. Specifically, the models offer the same predictions if and only if costs in the current contest models are multiplicatively separable in $p_{i}$ and $T$, which requires that costs are power functions $\Phi(i)=c_{i} t_{i}^{\gamma}$. We can then use Proposition 14 to study the inequality effects caused by a varying consumer income. Specifically, the results in Proposition 14 imply that whenever total income $\omega_{i}+S_{i}$ (endogenously) increases for all consumers, the inequality effects are entirely determined by the ratio $d V_{i} / V_{i}$; whenever this ratio decreases (increases) in $i$ over consumer types, an OR (IR) of income (and consumption) shares results. For example, a windfall gain in income, such that $d V_{i}=d V>0 \forall i$, must always cause more income and consumption equality in the sense of a monotonic IR. 
Idiosyncratic Changes We use the current setting to demonstrate that our inequality tools can be also applied in case of a purely idiosyncratic market condition. Suppose that $d V_{i}>0$ only for agent type $i$, while $d V_{j}=0$ for everybody else ${ }^{46}$ Note that a small change $d V_{i}>0$ will preserve the agent order as captured by $p(\cdot)$. To obtain the inequality effects, we simply need to evaluate (9) with the above valuation shock.

Proposition 15 If $\Phi\left(t_{i}\right)=c t_{i}^{\gamma}$, then the idiosyncratic change $d V_{i}>0$ increases $p_{i}$, while the $p_{j}$ for all other agent types decreases in proportion to each other.

Thus, all agents expect for $i$ lose, and the loss is most pronounced for the strongest agents.

Two Prizes We now study the inequality effects of introducing (or modifying) a second prize. This is of interest, e.g., to sport economics, where assuring a certain "competitive balance" between different teams is a central aspect of tournament design as this adds a desired element of unpredictability to such contests (Szymanski, 2003). More generally, our contribution differs from the existing literature on contest architecture, as we seek to elicit the full distributive effects of a change in the prize structure, rather than identifying the reward schemes that maximize the aggregate efforts or the winning effort.

Consider a contest with two fixed prizes $V_{1}>0$ and $V_{1} \geq V_{2} \geq 0$. To focus on the inequality effects induced by these prizes, we assume that cost functions are $\Phi\left(i, t_{i}\right)=c_{i} t_{i}^{\gamma}$, where $c_{i}$ is increasing over agent types ${ }^{47}$ The probability to win the first prize is $p_{i}=t_{i} / T$ while, by sampling with replacement, $\left(1-p_{i}\right) p_{i}$ is the chance of obtaining the second prize 48 These formulas only make sense by assuming a finite number $N$ of atomistic agents. Formally, the set of agents is $I \equiv\{1 / N, . ., n / N, \ldots ., 1\}$, such that $i \in[0,1]$ for any $i \in I$, and $p_{i} \in[0,1]$ is a probability mass function rather than a density 49 Expressed in terms of market shares, the payoff function thus is

$$
\Pi_{i}=p_{i} V_{1}+\left(1-p_{i}\right) p_{i} V_{2}-c(i)\left(p_{i} T\right)^{\gamma} .
$$

The following proposition shows how a change in the prize scheme $\left(V_{1}, V_{2}\right)$ affects the distribution of the chances $p_{i}$ to seize the first prize or any prize $w_{i} \equiv p_{i}+\left(1-p_{i}\right) p_{i}$.

Proposition 16 If $\frac{d V_{1}}{V_{1}}>(<) \frac{d V_{2}}{V_{2}}$ an $O R$ (IR) of the chance to win the first prize $p_{i}$ results. If $\frac{d V_{1}}{V_{1}}=\frac{d V_{2}}{V_{2}}, p_{i}$ is invariant to the allocation of prize money. The previous patterns also apply to the chance of winning any prize $w_{i}$, provided that $p_{i}<1 / 2 \forall i$.

Comparing Proposition 16 with its single-prize (Proposition 13) shows that, given the assumed cost function, a unilateral change of $V_{1}$ or $V_{2}$ only induces inequality effects in the model with

\footnotetext{
${ }^{46}$ In terms of the general setting from Section 3 , this formally corresponds to the case where $d V_{i}=$ $g_{x}(i, p(i), T ; x)>0$ and $d V_{j}=g_{x}(j, p(j), T, x)=0 \forall j \neq i$.

${ }^{47}$ As we know from previous results, this cost function works in a distributionally "neutral" way. It is straightforward to adjust our analysis for other cost functions.

${ }^{48} \mathrm{By}$ sampling with replacement, we mean that if agent $i$ does not win the first prize, for which the chance was $p_{i}=t_{i} / T$, then $i$ competes again with the same effort and the same agents for the second prize, i.e., the agent who won the first prize is not removed from the pool. Thus, the chance of $i$ to win the second prize, given that $i$ has not won the first prize, also corresponds to $t_{i} / T$.

${ }^{49}$ It is straightforward to verify that all inequality results from Section 3 that pertain to Class I densities also apply to the case where $p_{i}$ is a probability mass function defined over a given finite set of atomistic agents.
} 
two prizes, while the prize level was inequality preserving in the single-prize case. Moreover, the two prizes have diametrically opposite effects on inequality, as an increase of the first (second) prize implies more inequality (equality) in the first-prize winning chances $p_{i}$, and likely also in the overall winning chances $w_{i}$.

With respect to designing a balanced contests, such as a sports tournament, Proposition 16 suggests that if the overall prize money $V=V_{1}+V_{2}$ increases, then both prizes need to be increased proportionally $\left(d V_{1} / V 1=d V_{2} / V_{2}\right)$ for $p_{i}$ or $s_{i}$ to stay constant. Likewise, Proposition 16 shows how changing the composition of a given prize budget $V \equiv V_{1}+V_{2}>0$ affects the dispersion of success chances. Because $d V_{1}<0$ and $d V_{2}>0$ both imply an IR, ceteris paribus, it follows that an even prize split $V_{1}=V_{2}=V / 2$ must generate the most balanced contest, while setting $V_{1}=V$ (a single-prize contest) yields the most imbalanced contest. In addition, there is an effort-equality trade-off because aggregate effort $T\left(V_{1}, V_{2}\right)$ decreases if $V_{2}=V-V_{1}$ increases 50

Advertising for Attention A central aspect of advertising is the attraction of consumer attention. If attention is an exhaustible resource, the competition for attention takes on the form of a contest (Hefti, 2018). Besides attention-seeking, advertising can have persuasive effects that alter the willingness-to-pay of attentive consumers (Bagwell, 2007). We next study how these effects matter for the firm-side market shares.

Let $t_{i}$ quantify firm $i$ 's advertising intensity, which affects its market share $p_{i}=t_{i} / T$ due to attracting consumer attention, and possibly also the willingness-to-pay of attentive consumers. Regarding the latter, we set $V_{i}\left(t_{i}\right)=\alpha t_{i}+\beta$, where $V(\cdot)$ summarizes how much each firm earns from its attentive consumers. The parameter $\beta>0$ corresponds to a basic willingess-to-pay, while $\alpha \geq 0$ is a "rate of persuasion", capturing how advertising converts into revenue from attentive consumers; if $\alpha=0$, advertising only serves to attract attention.

Expressed as a competition for market shares, the payoff is

$$
\Pi_{i}=p_{i}\left(\alpha p_{i} T+\beta\right)-\Phi\left(i, p_{i} T\right)
$$

where $\Phi(\cdot)$ are the total advertising expenditures, and $c_{i}$ is increasing over agent types, such that $p(\cdot)$ is Class I. Formally, 35) is a non-trivial variant of 33 where the prize function $V(\cdot)=\alpha p_{i} T+\beta$ depends on $p_{i}$ and $T$.

To concentrate on the inequality effects implied by the two aspects of advertising, we let $\Phi(\cdot)=c_{i}\left(p_{i} T\right)^{\gamma}, \gamma \geq 2$, implying that the cost side has a neutral effect on market inequality. Further, we assume parameter values such that $t_{i}>1 \forall i$ in equilibrium 51 The parameter $\gamma$ quantifies how subtle the market shares respond individual changes of effort. In the present context, a larger value of $\gamma$ means that it is harder for the firms to influence consumer attention

\footnotetext{
${ }^{50}$ To see that $T\left(V_{1}, V-V_{1}\right)$ decreases in $V_{1}$, use $V_{2}=V-V_{1}$ in 49 and note that $\frac{\partial}{\partial V_{1}} p(i)>0$. This is related to Clark and Riis (1998), who consider the case of a multi-prize contest with symmetric contestants. Their main concern is about the aggregate effort, and they find that highest aggregate effort requires to award only one prize.

${ }^{51} \gamma \geq 2$ assures the strong quasiconcavity of $\Pi(i)$ in $p_{i}$. Further, the requirement that $t_{i}>1 \forall i$ simplifies the proof of Proposition 17 below, and can be always assured, e.g. if $c_{i}$ is sufficiently low or $\beta$ sufficiently high.
} 
to their favor.

The parameters $\alpha$ and $\gamma$ determine the role of advertising for extracting consumer budget. For instance, high values of $\gamma$ and $\alpha$ imply that advertising increases firm revenue basically by persuading attentive consumers, while it is utterly hard for firms to increase their amount of attentive consumers. By contrast, low values of $\gamma$ and $\alpha$ mean that a firm can best increase its revenues by competing harder for consumer attention, while the willingness-to-pay of the attentive consumers is mostly insensitive to advertising.

Proposition 17 An increase in the rate of persuasion $(d \alpha>0)$ induces an OR of $p_{i}$, while less attentional control $(d \gamma>0)$ induces an IR of $p_{i}$.

Proposition 17 offers two rationales how advertising may affect the market share dispersion. First, we can expect to observe an increasing market concentration if advertising works more persuasively. The intuition is that an increase in the worthiness of attracting attention must benefit the firms with the largest market shares by most. These firms therefore have the strongest incentive to increase the attention they receive. Second, market concentration must be higher if firms have more influence on their chances to attract attention. This occurs because firms with lower costs can better exploit their advantage the more sensitively consumer attention responds to advertising.

\subsection{Inequality Effects in Competition Models: A Synthesis}

While the competition models covered in Section 4 represent vastly different forms of competition, comparing the various propositions shows that highly similar inequality patterns appear in all these models. At the basic level, this follows because the different competition models show a similar formal structure of their respective and equilibrium equations.

This becomes most evident if the competition model is stated as a competition for market shares. It then is relatively easy to observe, e.g., that the firm-side competition for market shares in monopolistic or perfect competition models is equivalent, from the formal viewpoint, to a contest where the (endogenous) prize function is homogeneous across all agents. The underlying, common structure in these cases can best be described as a symmetric competition, in thus that the equilibrium value per unit of market share is identical to all firms.

In such a symmetric competition, the inequality effects induced by level variables depend entirely on the properties of the cost functions, while properties like shape or curvature of the marginal benefits play no role. More specifically, level variables are inequality preserving if and only if costs are given by a common-elastic Power Function $\Phi\left(i, t_{i}\right)=c_{i} t_{i}^{\gamma}$. Such cost functions are a natural choice of modeling in some circumstances, perhaps most prominently, in case of homogeneous production functions. Moreover, we established a tight connection between market and payoff shares if costs are given by power functions: These shares coincide if and only if all agents have a common-elastic power function. Taken together, the previous facts imply that, in terms of observables, level variables cause inequality effects if and only if market and payoff shares disagree.

The reason for these results is that the equilibrium forces equate marginal costs across all agents with a symmetric competition. By contrast, with a heterogeneous prize function, 
marginal costs are not equated in equilibrium, and then the properties of the prize functions can affect the inequality patterns. In our applications, such a situation arose with a twoprize contest, or if advertising has idiosyncratic effects on the willingness-to-pay. Moreover, the equilibrium conditions that determine consumption inequality in Section 4.2 .2 are formally equivalent to the ones generated by contests with heterogeneous prize functions, explaining why we observe similar inequality patterns in contests with heterogeneous prize functions.

The fact that the cost functions can be decisive for the possible inequality effects induced by level variables has specific ramifications, particularly in case of market competition. For example, some have argued that an increasing international integration has the key effect of increasing the total level of disposable consumer income. To explain the empirically observed increasing inequality on the firm side associated with such changes, the literature has put forth demand-side explanations (Mrázová and Neary, 2017), while our results emphasize a supply-side explanation.

Our analysis of monopolistic and perfect competition reveals that an increasing disposable income induces firm-side inequality effects if the firms differ in their returns to scale. This finding is robust in thus that it arises in any type of symmetric competition. Moreover, the prediction that growing income, or an increased industry-level productivity, leads to an increased market concentration fits the stylized observation that "Blockbusters", e.g., in the movie or music industry, have become more successful than ever (e.g., Elberse, 2008). Indeed, if one thinks of media giants such as Disney or Sony, it is likely that in such industries different returns to scale are a key aspect of the ex ante firm heterogeneity.

\section{Conclusion}

This article analyzes the equilibrium inequality effects that are caused by exogenous changes in presence of ex ante heterogeneous agents. We pursue this question using a novel approach that represents the competition the agents face as a competition for market shares. We see at least two merits offered by this approach. First, the formulation as a competition for market shares helps us to identify a common underlying structure in different models of competition, allowing for a unified perspective on the causes of inequality effects. Second, the inequality tools derived from this framework enable us to enlarge the existing knowledge about equilibrium inequality in the context of specific applications.

Regarding the former, we show that many competition models share the formal paradigm of a symmetric competition in thus that the marginal benefit per unit of market shares is identical for all agents. This clarifies why these models offer conceptually identical inequality predictions. In particular, if a market condition affects all agents identically - in which case we refer to it as a "level variable" - then the resulting inequality effects depend exclusively on the marginal costs of maintaining the respective market share. Such a level variable cannot induce any inequality effects if and only if all agents are subject to the same returns to scale in their cost functions. By contrast, an increase in a level variable increases the polarization in the market 
share dispersion, if the agents with the largest market shares also feature the most favorable returns to scale. The reason is that these agents can exploit their scale effects to adapt more profitably to the new situation.

Our inequality tools provided novel insights within the context of our applications, partly because we were able to derive the inequality effects exploiting a single equilibrium equation, rather than analyzing the full set of equilibrium equations, without relying on numerical methods, and without specifying the ex ante agent heterogeneity in full detail, e.g., in terms of a specific, parametrized distribution.

The analytical merits of our approach perhaps are perhaps most evident in our application to monopolistic competition. Our framework permits the inequality analysis to go substantially beyond the standard assumption of constant marginal costs on the firm side, while remaining analytically tractable. We establish that the ex ante dispersion of the scale effects in production have crucial implications for the inequality effects triggered by "level" variables, such as total income, industry-wide efficiency, or a sales tax. These variables do not affect the market and payoff share dispersions if and only if all firms are subject to the same returns to scale in production. By contrast, these variables induce inequality effects if firms differ in their returns to scale - a finding, which we deem particularly relevant for industries such as movie or music. It is well conceivable that companies as Disney or Sony feature far more favorable scale effects than smaller studios. In such a case, our analysis predicts that a growing consumer income or a common efficiency gain lead to quantity growth in the market paired with a rising inequality of the firm-side market and payoff shares. Such patterns fit with empirical evidence indicating that "Blockbuster firms" have become more successful over the last decades, and generally complements demand-side explanations for an increasing market inequality.

Besides monopolistic competition, we used our framework to study the inequality effects of common market conditions, such as a tax, within both market sides, thereby complementing the classical incidence literature that deals with the distribution of the tax burden between consumers and firms. In two other applications, we explore how inequality evolves in case of private ownership economies if consumers receive a windfall gain similar to an unconditional basic income, or if the propensity to consume relative to enjoying leisure changes. Finally, we also consider a set of applications inspired by IO, political economy and marketing featuring a "competition for prize", and highlight the key connections of such settings to our other models of market competition.

The inequality analysis developed in this article can be extended in various directions, some of which we already follow in our other applied work. Further, the inequality analysis may provide a useful stepping-stone for studying normative questions related to inequality. For example, an organizational planer may need to decide which instruments, wage schemes or prize structures to implement for obtaining a certain distributional outcome, e.g., due to fairness considerations. Understanding precisely the distributional consequences, say, of various policy instruments most likely constitutes a central milestone in dealing with such issues. Finally, the fact that on most 
occasions we were able to describe the inequality effects without specifying details of the ex-ante heterogeneity can be of interest to empirical work, as real-world agents barely are symmetric, but the precise extent of the heterogeneity may be unknown to an econometrician.

\section{References}

Acemoglu, D. and Jensen, M. K. (2010). Robust comparative statics in large static games. In 49th IEEE Conference on Decision and Control, pages 3133-3139. IEEE.

Acemoglu, D. and Jensen, M. K. (2013). Aggregate comparative statics. Games and Economic Behavior, $81: 27-49$.

Aguiar, M. and Bils, M. (2015). Has consumption inequality mirrored income inequality? American Economic Review, 105(9):2725-56.

Aguiar, M. and Hurst, E. (2007). Measuring trends in leisure: the allocation of time over five decades. The Quarterly Journal of Economics, 122(3):969-1006.

Alos-Ferrer, C. and Ania, A. B. (2005). The evolutionary stability of perfectly competitive behavior. Economic Theory, 26:497-516.

Amegashie, J. A. (1999). The number of rent-seekers and aggregate rent-seeking expenditures: an unpleasant result. Public Choice, 99(1-2):57-62.

Anderson, S. P., De Palma, A., and Thisse, J. F. (1992). Discrete choice theory of product differentiation. MIT press.

Atkinson, A. (1970). On the measurement of inequality. Journal of Economic Theory, 2(3):244-263.

Attanasio, O. P. and Pistaferri, L. (2016). Consumption inequality. Journal of Economic Perspectives, $30(2): 3-28$.

Bagwell, K. (2007). The economic analysis of advertising. In Armstrong, M. and Porter, R., editors, Handbook of Industrial Organization, volume 3. Amsterdan: Elsevier B.V.

Blanchard, O. (2004). The economic future of europe. Journal of Economic Perspectives, 18(4):3-26.

Camacho, C., Kamihigashi, T., and Sağlam, Ç. (2018). Robust comparative statics for non-monotone shocks in large aggregative games. Journal of Economic Theory, 174:288-299.

Casella, G. and Berger, R. L. (2002). Statistical inference, volume 2. Duxbury Pacific Grove, CA.

Chung, T. Y. (1996). Rent-seeking contest when the prize increases with aggregate efforts. Public Choice, $87: 55-66$.

Clark, D. J. and Riis, C. (1998). Competition over more than one prize. The American Economic Review, 88(1):276-289.

Corchon, L. C. (1994). Comparative statics for aggregative games: The strong concavity case. Mathematical Social Sciences, 28(3):151-165.

Cornes, R. and Hartley, R. (2012). Fully aggregative games. Economics Letters, 116(3):631-633.

Dixit, A. K. and Stiglitz, J. E. (1977). Monopolistic competition and optimum product diversity. The American Economic Review, 67:297-308.

Elberse, A. (2008). Should you invest in the long tail? Harvard Business Review, 86(7/8):88-96.

Ellison, G. and Wolitzky, A. (2012). A search cost model of obfuscation. The RAND Journal of Economics, 43(3):417-441.

Gervais, A. (2015). Product quality and firm heterogeneity in international trade. Canadian Journal of Economics, 48(3):1152-1174.

Heathcote, J., Storesletten, K., and Violante, G. L. (2014). Consumption and labor supply with partial insurance: An analytical framework. American Economic Review, 104(7):2075-2126. 
Hefti, A. (2016). On the relationship between uniqueness and stability in sum-aggregative, symmetric and general differentiable games. Mathematical Social Sciences, 80:83-96.

Hefti, A. (2018). Limited attention, competition and welfare. Journal of Economic Theory, 178.

Hefti, A., Grossmann, M., and Shen, P. (2020). Heterogeneity and prize structure in dynamic contests. Working paper.

Hefti, A. and Lareida, J. (2020). Competitive attention, superstars and the long tail. Working Paper.

Jensen, M. K. (2017). Distributional comparative statics. The Review of Economic Studies, 85(1):581610.

Konrad, K. (2009). Strategy and Dynamics in Contests. Oxford University Press.

Maoz, Y. D. (2010). Labor hours in the united states and europe: The role of different leisure preferences. Macroeconomic Dynamics, 14(2):231.

Melitz, M. J. (2003). The impact of trade on intra-industry reallocations and aggregate industry productivity. Econometrica, 71(6):1695-1725.

Mieszkowski, P. (1969). Tax incidence theory: The effects of taxes on the distribution of income. Journal of Economic Literature, 7(4):1103-1124.

Morris, S. and Shin, H. S. (2002). Social value of public information. American Economic Review, 92(5):1521-1534.

Mrázová, M. and Neary, J. P. (2017). Not so demanding: Demand structure and firm behavior. American Economic Review, 107(12):3835-74.

Mrázová, M., Neary, P. J., and Parenti, M. (2016). Sales and markup dispersion: theory and empirics. Working paper.

Newman, M. E. (2005). Power laws, pareto distributions and zipf's law. Contemporary physics, 46(5):323351.

Posner, R. A. (1992). Economic analysis of law. Boston, Toronto and London: Little, Brown and Company.

Redding, S. (2011). Theories of heterogeneous firms and trade. Annual Review of Economics, 3:77-105.

Szymanski, S. (2003). The economic design of sporting contests. Journal of economic literature, 41(4):1137-1187.

Topkis, D. M. (1998). Supermodularity and Complementarity. Princeton: University Press. 


\section{A Proofs}

Proof of Proposition 1 We first prove that $p(i)>(\geq) p(j)$ if $j>i$. Let $p>0$ and note that $p(i) \gtreqless p \Leftrightarrow g(i, p, T) \gtreqless \varphi(i, p, T)$ because, by strong quasiconcavity (A1), $g(i, \cdot, T)$ must intersect $\varphi(i, \cdot, T)$ from above at $p(i)$ (see Figure 3, Appendix 3, for an illustration). Further, in equilibrium

$$
g(i, p(j), T)>(\geq) g(j, p(j), T)=\varphi(j, p(j), T)>(\geq) \varphi(i, p(j), T)
$$

By Assumption 2 and (5). Hence $g(i, p(j), T) \geq \varphi(i, p(j), T)$ and thus $p(i) \geq p(j)$, where these two inequalities are strict if at least one inequality in $(36)$ is strict. It also follows that $p(i)=p(j)$ if all inequalities in (36) are equalities, which proves the last claim of Proposition 1. Let $B(i, p, T) \equiv p V(i, p, T)$. The claims about payoffs holds because

$$
\begin{aligned}
\Pi(i) & =B(i, p(i), T)-\Phi(i, p(i), T) \\
& \geq B(i, p(j), T)-\Phi(i, p(j), T)>(\geq) B(j, p(j), T)-\Phi(j, p(j), T)=\Pi(j),
\end{aligned}
$$

where the first inequality follows from optimality and the second from Assumption 2 .

Proof of Lemma 1 For each $i \in I$, define $z\left(i, p, T ; x_{0}\right) \equiv \frac{g\left(i, p(i), T ; x_{0}\right)}{\left.\varphi(i, p(i), T) ; x_{0}\right)}$. Thus, using shorthand notation, (7) is $z(i)=z(j)$. Moreover, (5) implies that $z(i)=1 \forall i$ in equilibrium. Total differentiation of this equation yields

$$
d p(i) z_{p}(i)=d p(j) z_{p}(j)+r, \quad r \equiv\left(z_{T}(j)-z_{T}(i)\right) d T+\left(z_{x}(j)-z_{x}(i)\right) d x .
$$

Defining $\kappa_{i} \equiv z_{p}(i) p(i)$ and $\Delta_{i} \equiv \frac{d p(i)}{p\left(i ; x_{0}\right)}$, the previous equation gives

$$
\Delta_{i}=\Delta_{j} \frac{\kappa_{j}}{\kappa_{i}}+\frac{1}{\kappa_{i}} r
$$

Noting that

$r=\left(\left(\frac{g_{T}(j)}{g(j)}-\frac{\varphi_{T}(j)}{\varphi(j)}\right) d T+\left(\frac{g_{x}(j)}{g(j)}-\frac{\varphi_{x}(j)}{\varphi(j)}\right) d x\right)-\left(\left(\frac{g_{T}(i)}{g(i)}-\frac{\varphi_{T}(i)}{\varphi(i)}\right) d T+\left(\frac{g_{x}(i)}{g(i)}-\frac{\varphi_{x}(i)}{\varphi(i)}\right) d x\right)$

and

$$
\kappa_{i}=z_{p}(i) p(i)=\frac{g_{p}(i) p(i)}{g(i)}-\frac{\varphi_{p}(i) p(i)}{\varphi(i)}=\varepsilon_{i}-\eta_{i}
$$

(37) becomes

$$
\Delta_{i}=\Delta_{j} \frac{\eta_{j}-\varepsilon_{j}}{\eta_{i}-\varepsilon_{i}}+\frac{1}{\eta_{i}-\varepsilon_{i}}(-r)=\Delta_{j} k_{i j}+R_{i j}
$$

which yields $(9)$. The claims that $\eta_{i}>0$ and $\varepsilon_{i}<\eta_{i}$ follow from strong quasiconcavity (A1) in Assumption 1 
Proof of Theorem 1 We prove the first claim by contradiction. Hence suppose that $R=0$ $\forall i, j \in[0,1]$ and any $x \in X$, but $\exists j \in(0,1)$ such that $\Delta_{j} \neq 0$ (equivalently $d p(j) \neq 0$ ). Because in equilibrium the condition

$$
\int \frac{\partial p(s ; x)}{\partial x} d s=0
$$

must hold, we can then suppose, wlog, that $\Delta_{j}>0$ for some $j \in(0,1)$. By $(9)$ we must have $\Delta_{i}>0$ for all $i<j$, and because of $(38) \exists j^{\prime} \in(0,1), j^{\prime}>j$, such that $\Delta_{i}<0$ for all $i>j^{\prime}$. Take $i<j$ and $i^{\prime}>j^{\prime}$. Then $\Delta_{i}>0$ but also $\Delta_{i}=k_{i i^{\prime}} \Delta_{i^{\prime}}<0$, contradiction. Turning to the second claim, note that if $R \neq 0$ for some $i, j$ then $\Delta_{i}=0 \forall i \in[0,1]$ is impossible by (9). Hence $\forall x \in X \exists i: \Delta_{i}(x) \neq 0$, or equivalently $\frac{\partial p(i ; x)}{\partial x} \neq 0$, and therefore $\exists \delta>0$ such that $p\left(i ; x^{\prime}\right) \neq p(i ; x)$ for $x^{\prime} \in(x-\delta, x+\delta)$.

Proof of Corollary 1 Because $x$ is a level variable and $\varphi(i)$ is independent of $x$,

$$
A(i)-A(j)=\left(\frac{\varphi_{T}(j)}{\varphi(j)}-\frac{\varphi_{T}(i)}{\varphi(i)}\right) T^{\prime}(x)
$$

Multiplicative separability, $\varphi(i, p, T)=u(i, p) v(T)$, then implies that $A(i)-A(j)=0$, and thus $R_{i j}=0 \forall i, j$ by (10). This shows that $x$ is inequality preserving by Theorem 1 . For the converse, we need that $R_{i j}=0 \forall x \in X$ if $x$ is inequality preserving by Theorem 1 . Thus, as $T^{\prime}(x) \neq 0$, we must have that $A(i)=A(j) \forall i, j$ or, equivalently, that $\frac{\varphi_{T}(i, p, T)}{\varphi(i, p, T)}=h(T) \forall i, p, T$. Integrating this equation with respect to $T$ shows that then $\varphi(i, p, T)$ must be of the form $\varphi(i, p, T)=u(i, p) v(T)$.

Proof of Corollary 2 If $\Phi(i, t)=c(i) t^{\gamma}$, then $\varphi(i, p(i), T)=\gamma c(i) p(i)^{\gamma-1} T^{\gamma}$ in the transformed model, showing the multiplicative separability of $\varphi(i)$ in $(i, p)$ and $T$, and the first claim follows from Corollary 1. For the converse, note that

$$
\varphi(i) \equiv \frac{\partial \Phi(i, p(i) T)}{\partial p(i)}=h(i, p(i) T) T
$$

where $h(i, p(i) T) \equiv \Phi_{t}(i, p(i) T)$. Then

$$
\frac{\varphi_{T}(i)}{\varphi(i)}=\frac{h_{t}(i, p(i) T) p(i)}{h(i, p(i) T)}+\frac{1}{T}
$$

where $h_{t}$ denotes the partial derivative with respect to the 2 nd argument of $h(i, \cdot)$. If the level variable $x$ is inequality preserving, then $R=0$ everywhere by Theorem 1 Because $x$ is a level variable and $T^{\prime}(x) \neq 0, R_{i j}=0$ necessarily requires that $\frac{\varphi_{T}(i)}{\varphi(i)}=\frac{\varphi_{T}(j)}{\varphi(j)}$ by $(10)$. But the last equation is equivalent to $\frac{h_{t}(i, t(i)) t(i)}{h(i, t(i))}=\frac{h_{t}(j, t(j)) t(j)}{h(j, t(j))} \forall i, j$ by $(39)$, meaning that $\frac{h_{t}(i, t(i)) t(i)}{h(i, t(i))}$ 
must be equal to a fixed constant $\chi, \forall i$. But this implies that $h(i, t(i))$ must be of the form $h(i, t(i))=w(i) t(i)^{\chi}$, and the fact that $\int h(i, t) d t=\Phi(i, t)$ gives $\Phi(i, t(i))=\frac{w(i)}{\chi+1} t(i)^{\chi+1}$, proving the second claim.

Proof of Theorem 2 Step 1: We prove the first claim, restricting attention to the ORcase (the IR-case is similar). Because $R\left(x_{0}\right)$ is uniformly positive, $\exists i \in(0,1): \Delta_{i}\left(x_{0}\right)>0$ by the proof of Theorem 1. By the integral condition (38), there then must also be $i^{\prime} \in(0,1)$ : $\Delta_{i^{\prime}}\left(x_{0}\right)<0$. It then follows from $(9)$ that $i_{0}=\sup \left\{i \in[0,1]: \Delta_{i}\left(x_{0}\right)>0\right\} \in(0,1), i_{1}=\inf \{i \in$ $\left.[0,1]: \Delta_{i}\left(x_{0}\right)<0\right\} \in(0,1)$ and $i_{0} \leq i_{1}$. For any $i<i_{0}: \Delta_{i}\left(x_{0}\right)>0$ and hence $\frac{\partial p\left(i ; x_{0}\right)}{\partial x}>0$. This derivative condition implies that $\forall i<i_{0} \exists \delta_{i}>0: p(i ; x)>p\left(i ; x_{0}\right) \forall x \in\left(x_{0}, x_{0}+\delta_{i}\right)$.

Step 2: Because $p(\cdot ; x)$ is Class I, there is a finite number of equivalence classes to the left of $i_{0}$, and we only need to consider a single $i$, with corresponding $\delta_{i}$, for each step of $p(\cdot ; x)$ to the left of $i_{0}$. Let $\delta^{0}>0$ be the smallest value of these $\delta_{i}$. We have thus shown that $\exists i_{0} \in(0,1)$ such that for any given $x \in\left(x_{0}, x_{0}+\delta^{0}\right)$ we have $p(i ; x)>p\left(i ; x_{0}\right)$ for $i<i_{0}$. A similar argument shows that we can find $\delta^{1}>0$ such that $\exists i_{1} \in(0,1)$ such that $p(i ; x)<p\left(i ; x_{0}\right)$ for $i>i_{1}$ and any $x \in\left(x_{0}, x_{0}+\delta^{1}\right)$. Let $\delta \equiv \min \left\{\delta^{0}, \delta^{1}\right\}>0$. Summarizing, the arguments so far show that $\exists i_{0}, i_{1} \in(0,1), i_{0} \leq i_{1}$ such that for $x \in\left(x_{0}, x_{0}+\delta\right)$ we have $p(i ; x)>p\left(i ; x_{0}\right)$ for $i<i_{0}$ and $p(i ; x)<p\left(i ; x_{0}\right)$ for $i>i_{1}$. If $\Delta_{i} \neq 0$ for any $i \in\left(i_{0}, i_{1}\right]$ we must have $i_{0}=i_{1}$ and the proof is complete. Now suppose that $\exists m \in\left(i_{0}, i_{1}\right]: \Delta_{m}\left(x_{0}\right)=0$. Then $(9)$ implies that $\Delta_{i}>0$ for any $m \triangleright i$, and $\Delta_{j}<0$ for any $j \triangleright m$. But this shows that there can be at most one step of $p(\cdot ; x)$ for which $\Delta_{m}\left(x_{0}\right)=0$. It follows that independent of whether $p\left(m ; x^{\prime}\right)>=<p(m ; x)$ for $x \in\left(x_{0}, x_{0}+\delta\right), p\left(\cdot ; x^{\prime}\right)$ must be OR of $p\left(\cdot ; x_{0}\right)$.

We now prove the second claim. By step 1 and the global uniform positivity of $R$, we must have $\Delta_{0}(x)>0$ and thus $\frac{\partial p(0 ; x)}{\partial x}>0$ for any $x>x_{0}$ (note that this result is valid also if $p(\cdot)$ is of Class II), hence $p\left(i ; x^{\prime}\right)>p\left(i ; x_{0}\right) \forall i \in[0]$. Similarly, $\Delta_{1}(x)<0$ for all $x>x_{0}$, hence $p\left(i ; x^{\prime}\right)<p\left(i ; x_{0}\right) \forall i \in[1]$.

Proof of Corollary 3 In text.

Proof of Corollary 4 In text.

Proof Lemma $2 \quad u^{\prime} v \geq u v^{\prime}$ hence also $u^{\prime} v-u v \geq u v^{\prime}-u v$ or $u^{\prime}-u \geq \frac{u}{v}\left(v^{\prime}-v\right)>v^{\prime}-v$.

Proof of Theorem 3 We only prove the OR case, and show that (16) implies condition (15). Noting the equivalence between (16] and (16), we define $f(x ; i, j) \equiv \frac{p(i ; x)}{p(j ; x)}$. If $p(\cdot)$ is Class II and 
16] is satisfied, then $f(x ; i, j)>f\left(x_{0} ; i, j\right)$ whenever $x>x_{0}$, and the claim follows from the fact that condition (15) induces a monotonic rotation. If $p(\cdot)$ is Class I, then $p(\cdot ; x)$ is piecewise constant for any given $x \in X$, with a finite number of downward jumps. If (16) is satisfied for any two $i, j \in(0,1)$ with $j \triangleright i$ that are not discontinuities of $p(\cdot ; x)$, then $f(x ; i, j)>f\left(x_{0} ; i, j\right)$ follows for any such $i, j$ and any $x>x_{0}$, proving the claim also for Class I densities.

Proof of Corollary 5 Define $d p(i ; x) \equiv \frac{\partial p(i ; x)}{\partial x}$. If $x$ is inequality preserving, then we must have $d p(i ; x)=0 \forall i \in I$ and $\forall x \in X$. But as

$$
\frac{\partial}{\partial x}\left(\frac{p(i ; x)}{p(j ; x)}\right)=0 \quad \Leftrightarrow \quad \frac{d p(i ; x)}{p(i ; x)}=\frac{d p(j ; x)}{p(j ; x)}
$$

we immediately obtain that $\frac{\partial}{\partial x}\left(\frac{p(i ; x)}{p(j ; x)}\right)=0, \forall i, j \in I$ and $\forall x \in X$ whenever $x$ is inequality preserving. In the other direction, suppose that $\frac{\partial}{\partial x}\left(\frac{p(i ; x)}{p(j ; x)}\right)=0 \forall i, j \in I$ and $\forall x \in X$ applies. Then, (40) implies that $\frac{d p(i ; x)}{p(i ; x)}=k(x)>0 \forall i \in I$, or equivalently $d p(i ; x)=k(x) p(i ; x)$. Because $p(\cdot ; x)$ is a density, it follows that $\int_{I} d p(s ; x) d s=0$ as well as $\int_{I} p(s ; x) d s=1$. Integrating $d p(i ; x)=k(x) p(i ; x)$ on both sides delivers that $k(x)=0$, which assures that $d p(i ; x)=0$ $\forall i \in I$ and any $x \in X$, meaning that $x$ must be inequality preserving.

Proof of Proposition 2 We only prove the first claim as the remaining claims are proved identically. Recall from the equivalence class argument in step 2 of the proof of Theorem 2 that, because $R$ is uniformly positive, there is a finite number of leading agents with $\Delta_{i}\left(x_{0}\right)>0$, possibly a single $\Delta_{m}\left(x_{0}\right)=0$ and a finite number of weaker agents with $\Delta_{j}\left(x_{0}\right)<0$. Define $f(i, j, x)=\frac{p(i, x)}{p(j, x)}$. If $\Delta_{i^{\prime}}\left(x_{0}\right) \geq 0$ then any $i$ with $i^{\prime} \triangleright i$ has $\Delta_{i}\left(x_{0}\right)>\Delta_{i^{\prime}}\left(x_{0}\right)$ by (9) and the fact that $k_{i j} \geq 1$. Hence we must have $\frac{\partial f\left(i, i^{\prime}, x_{0}\right)}{\partial x}>0$. If $\Delta_{i^{\prime}}\left(x_{0}\right)<0$ but $\Delta_{i}\left(x_{0}\right)>0$, then obviously $\frac{\partial f\left(i, i^{\prime}, x_{0}\right)}{\partial x}>0$ Thus for any pair $\left(i, i^{\prime}\right)$ as described above $\exists \delta_{i, i^{\prime}}>0$ such that $f\left(i, i^{\prime}, x^{\prime}\right)>f\left(i, i^{\prime}, x_{0}\right)$ for all $x^{\prime} \in\left(x_{0}, x_{0}+\delta_{i, i^{\prime}}\right)$. The proof is completed by letting $\delta>0$ be the smallest among these (finitely many) $\delta_{i, i^{\prime}}$ and $\delta^{0}, \delta^{1}$ as in the proof of Theorem 2 .

Proof of Theorem 4 The claim follows from Theorem 3 because, by (9), if $R(x)$ is globally uniformly positive (negative) and $k=1$, then $\Delta_{i}(x)>(<) \Delta_{j}(x)$, for any $j \triangleright i$ and any $x \in X$, and hence condition 16 holds.

Proof of Corollary 6 Let $j \triangleright i$ and $T^{\prime}(x)>0$. The claims follow from noting that $R_{i j}>(<) 0$ $\Leftrightarrow \psi(j)>(<) \psi(i) \Leftrightarrow k_{i j}>(<) 1$. 
Proof of Corollary 7 Because $g(i)$ and $\varphi(i)$ both are multiplicatively separable in $i, p$ and $(T, x), \varepsilon(i)$ and $\eta(i)$ in (8) must be independent of $i$, and thus $k=1$ uniformly by (9). Because $R>(<) 0$ uniformly if $\gamma_{i}<(>) \gamma_{j}$ by Corollary 3 , the claim follows from Theorem 4 .

Proof of Proposition $3 \quad(19)$ implies that $\eta_{i}, \varepsilon_{i}$ both are invariant to the agent index $i$; hence $k_{i j}=1$ for all $i, j \in I$ follows from (9). The remaining claim follows directly from Theorem 4 .

Proof of Proposition 4 Recall from Section 3.2.4 that if $h(i ; x)$ is a decreasing density with support $I$, then $x$ is inequality preserving iff the ratio $\frac{h(i ; x)}{h(j ; x)}$ is invariant to $x$ for all $i, j \in I$, and $2)$. Further, $x$ induces a monotonic OR (IR) of $h(\cdot ; x)$ whenever the ratio $\frac{h(i ; x)}{h(j ; x)}$ has a strictly positive $x$-derivative (Theorem 3).

Next, note that the ratio in 20$)$ is of the form $\frac{p(i)}{p(j)}=(m(i, j, x) v(i, j, x))^{\frac{1}{\beta-\alpha}}$, where $m(i, j, x) \equiv$ $\frac{\hat{g}(i, T ; x)}{\hat{g}(j, T ; x)}$ and $v(i, j, x) \equiv \frac{\hat{\varphi}(j, T)}{\hat{\varphi}(i, T)}$. Denoting partial derivatives with respect to $x$ as $d m(i, j, x)$ and $d v(i, j, x)$, respectively, it follows that $\frac{\partial}{\partial x} \frac{p(i)}{p(j)}>0$ iff $\frac{d m(i, j, x)}{m(i, j, x)}+\frac{d v(i, j, x)}{v(i, j, x)}>0$. Further, 22 shows that the ratio $\frac{s(i)}{s(j)}$ is of the form $\frac{s(i)}{s(j)}=\left(m(i, j, x)^{\beta} v(i, j, x)^{\alpha}\right)^{\frac{1}{\beta-\alpha}}$, and $\frac{\partial}{\partial x} \frac{s(i)}{s(j)}>0$ iff $\beta \frac{d m(i, j, x)}{m(i, j, x)}+\alpha \frac{d v(i, j, x)}{v(i, j, x)}>0$. Now, if $\hat{g}(i, T ; x)$ actually is invariant to the agent index $i$, ceteris paribus, then $m(i, j, x)=1$. Thus $d m(i, j, x)=0$ in this case and the claim in Proposition 4 The same argument proves the claim if instead $\hat{\varphi}(i, T)$ is invariant to $i$. The remaining claim follows directly from the above derivations as well.

Proof of Lemma 3 For the first claim, to see the "if" (the "only if" is obvious), suppose by contradiction that, wlog, there is $i$ for which $p(i)>s(i)$. But because $\frac{p(i)}{s(i)}=\frac{p(j)}{s(j)} \forall j \neq i$, it follows that also $p(j)>s(j) \forall j \neq i$, which is impossible as both densities must integrate to one. Next, suppose that $\frac{s(i)}{s(j)}>\frac{p(i)}{p(j)} \forall j \triangleright i$. Because $s(\cdot)$ and $p(\cdot)$ both are densities with the same support and the same equivalence classes $[i]$, it follows by the the logic of Proposition B.1 (Appendix B.4.1) and Defintion 9 that $s(\cdot)$ must be a monotonic OR of $p(\cdot)$ (and similar for the IR case).

Proof of Proposition 5 The fact that $I, \theta$ and $r$ are inequality preserving is stated in the main text. Further (27) implies that

$$
\frac{p_{i}}{p_{j}}=\left(\frac{c_{j}}{c_{i}}\right)^{\frac{\eta-1}{\eta(\gamma-1)+1}}\left(\frac{r_{i}}{r_{j}}\right)^{\frac{\gamma \eta}{\eta(\gamma-1)+1}} .
$$

Note that $p_{i}>p_{j}$ for any $j \triangleright i$ as $w_{i}$ is increasing over agent types. 411) yields the 3rd column of Table 1, which implies the 1st column by Theorem 3 and Corollary 5 . Note that in equilibrium 
we can write $\Pi_{i}=p_{i} I\left(\frac{\gamma \eta-\eta+1}{\gamma \eta}\right)$, which directly gives the fourth column of Table 1 and, by integration, also the last column. For payoff shares, note that $\frac{\Pi_{i}}{\Pi_{j}}=\frac{p_{i}}{p_{j}}=\frac{s_{i}}{s_{j}}$, which assures that $p_{i}=s_{i} \forall i$ by Lemma 3 , and the second column of Table 1 follows from the first column. From $p_{i}=\frac{r_{i}^{\eta} P_{i}^{1-\eta}}{T}$ we obtain

$$
\frac{P_{i}}{P_{j}}=\left(\frac{c_{i}}{c_{j}}\right)^{\frac{1}{1+(\gamma-1) \eta}}\left(\frac{r_{i}}{r_{j}}\right)^{\frac{(\gamma-1) \eta}{1+(\gamma-1) \eta}},
$$

and the fifth column follows from differentiating this expression. Finally, the Power Law property assures that all rotations must be monotonic by Proposition 3 .

Proof of Proposition 6 Let $j \triangleright i$, such that $\gamma_{j}>\gamma_{i}$. Evaluating (10) for 27) gives

$$
\operatorname{sign}\left(R_{i j}\right)=\operatorname{sign}(A(i)-A(j))=\operatorname{sign}\left(\left(\gamma_{j}-\gamma_{i}\right) \frac{T^{\prime}(\theta)}{T}\right), \quad j \triangleright i,
$$

Further, 27) implies that $\frac{\partial p(i ; T)}{\partial \theta}>0$ and $\frac{\partial p(i ; T)}{\partial T}<0$ for any fixed $T>0$, which assures that $T^{\prime}(\theta)>0$ by the procedure in Section 3.3 . This jointly with 42 shows that $R_{i j}$ is uniformly positive, which by Theorem 2 implies that $d \theta>0$ induces an OR of $p(\cdot)$. Using the fact that

$$
q_{i}=I p_{i}^{\frac{\eta}{\eta-1}} T^{\frac{1}{\eta-1}}
$$

in (27) and rearranging yields

$$
\frac{\eta-1}{\eta}\left(\frac{I}{T}\right)^{\frac{1}{\eta}}=\frac{\gamma_{i}}{\theta} q_{i}^{\left(\gamma_{i}-1\right)+\frac{1}{\eta}}
$$

and thus

$$
\frac{q_{i}^{\left(\gamma_{i}-1\right)+\frac{1}{\eta}}}{q_{j}^{\left(\gamma_{j}-1\right)+\frac{1}{\eta}}}=\frac{\gamma_{j}}{\gamma_{i}}
$$

Because $d \theta>0$ implies $d T>0$ and triggers an OR, (43) implies that $d q_{i}>0$ at least for some $i$ and, because of (45) $d q_{i}>0$ must apply $\forall i \in[0,1]$. Because $\theta$ does not enter (45) directly and $\gamma_{j}>\gamma_{i}$, the fact $d q_{i}>0 \forall i$ implies that also the ratio $\frac{q_{i}}{q_{j}}$ must increase for any given $j \triangleright i{ }^{52}$ By 430 , we further obtain that $\frac{q_{i}}{q_{j}}=\left(\frac{p_{i}}{p_{j}}\right)^{\frac{\eta}{\eta-1}}$, showing that also the ratio $\frac{p_{i}}{p_{j}}$ increases, which implies that the OR of $p(\cdot)$ must be monotonic by Theorem 3 . Likewise, the fact that $p_{i} T=P_{i}^{1-\eta}$ implies $\frac{p_{i}}{p_{j}}=\left(\frac{P_{j}}{P_{i}}\right)^{\eta-1}$ in equilibrium, which shows that relative prices must also increase. We now show that $d\left(p_{i} T\right)>0$, which implies that all prices $P_{i}$ must decrease. By 43), the fact that $d q_{i}>0$ implies $\eta \Delta_{i}+\frac{d T}{T}>0 \forall i \in I$, and hence also $d\left(p_{i} T\right)>0$ (recall that $\left.T^{\prime}(\theta)>0\right)$.

\footnotetext{
${ }^{52}$ Recall that we assume parameters to be such that $q_{i}>1 \forall i$.
} 
Equilibrium payoffs are $\Pi_{i}=p_{i} I\left(\frac{\left(\gamma_{i}-1\right) \eta+1}{\gamma_{i} \eta}\right)$, showing that $\Pi_{i}$ qualitatively behaves like $p_{i}$ as $\theta$ changes. Because $p(\cdot)$ displays an $\mathrm{OR}$, this further implies that winners and losers coexist, and a firm $i$ is a winner iff $p_{i}$ increases. Further, the ratio $\frac{\Pi_{i}}{\Pi_{j}}$ increases because $\frac{p_{i}}{p_{j}}$ does so as well. Let $v_{i} \equiv \frac{\left(\gamma_{i}-1\right) \eta+1}{\gamma_{i} \eta}$ and note that $v_{i}$ is strictly increasing over agent types. Aggregate payoff then is $\Pi(\theta)=\int p_{s} v_{s} I d s$. Because $d \theta>0$ induces an OR, there is $i_{0} \in(0,1)$ such that $d p_{i} \geq 0 \forall i \in\left(0, i_{0}\right)$ and $d p_{i}<0 \forall i \in\left(i_{0}, 1\right)$, where $\int_{0}^{i_{0}} d p_{s} d s=-\int_{i_{0}}^{1} d p_{s} d s$. Then $\Pi^{\prime}(\theta)=\int_{0}^{i_{0}} d p_{s} v_{s} d s+\int_{i_{0}}^{1} d p_{s} v_{s} d s$, and the claim that $\Pi^{\prime}(\theta)>0$ follows from

$$
\int_{0}^{i_{0}} d p_{s} v_{s} d s \leq \int_{0}^{i_{0}} d p_{s} v_{i_{0}} d s=-\int_{i_{0}}^{1} d p_{s} v_{i_{0}} d s<-\int_{i_{0}}^{1} d p_{s} v_{s} d s
$$

Finally, payoff shares are $s_{i}=\frac{\Pi_{i}}{\Pi}$, and thus $\frac{s_{i}}{s_{j}}=\frac{p_{i}}{p_{j}} \frac{v_{i}}{v_{j}}$. Because $\frac{p_{i}}{p_{j}}$ strictly increases, so does $\frac{s_{i}}{s_{j}}$, which by Theorem 3 assures that $s(\cdot)$ displays a monotonic OR.

Proof of Proposition 7 Let $j \triangleright i$. Using the procedure outlined in Section 3.3 , it is straightforward to verify that $T^{\prime}(I)<0$. Then (44) implies that $d q_{i}>0 \forall i \in[0,1]$ for $d I>0$. Because $I$ does not enter (45) directly and $\gamma_{j}>\gamma_{i}$, the fact that $d q_{i}>0 \forall i \in I$ further implies that the ratio $\frac{q_{i}}{q_{j}}$ strictly increases for $d I>0$. Therefore, 43 assures that also $\frac{p_{i}}{p_{j}}$ increases for $d I>0$ by which, by Theorem 3 implies a monotonic OR of $p(\cdot)$. Since $\Pi_{i}=p_{i} I\left(\frac{\gamma_{i} \eta-\eta+1}{\gamma_{i} \eta}\right)$, relative profits increase in $I$, and because $p_{i} T=P_{i}^{1-\eta}$ so do relative prices $\frac{P_{j}}{P_{i}}$. Finally, the fact that $\frac{p_{i}}{p_{j}}$ increases also assures that $\frac{s(i)}{s(j)}$ must increase, which implies that $s(\cdot)$ must also display a monotonic OR by Theorem 3 .

Proof of Proposition 8 Applying the procedure from Section 3.3 shows that $T^{\prime}(x)>0$ because $P_{T}(T ; x)<0, P_{x}(T ; x)>0$ and $\varphi_{q}(i, q)>0$ by strict convexity. Then, the first claim follows from Corollary 2, and the last claim from Corollary 6. Regarding the effect of $x$ on $P$ and $q_{i}$, note that as $T^{\prime}(x)>0$ we must have $q_{i}^{\prime}(x)>0$ for some $i$. But because in equilibrium also $\varphi\left(i, q_{i}\right)=\varphi\left(j, q_{j}\right)$ by $(29)$, strict convexity implies that $q_{i}^{\prime}(x)>0 \forall i$. Moreover, 29) then also implies that $d P=P_{T}(T(x) ; x) T^{\prime}(x)+P_{x}(T(x) ; x)>0$, proving the remaining claim.

Proof of Proposition 9 Let $j \triangleright i$, and note that $\Pi_{i}=p_{i} T P(T ; x) \frac{\psi_{i}-1}{\psi_{i}}$. It follows that $\frac{p_{i}}{p_{j}}=$ $\frac{s_{i}}{s_{j}} \frac{\psi_{i}}{\psi_{j}} \frac{\psi_{j}-1}{\psi_{i}-1}$. This shows that $\frac{p_{i}}{p_{j}}=\frac{s_{i}}{s_{j}} \forall i, j$ if and only if $\psi_{j}=\psi_{i} \forall i, j$, meaning that costs must be of the form $\Phi(i, q)=c_{i} q^{\gamma}$, and the first claim follows from Lemma 3 . Moreover, $\frac{p_{i}}{p_{j}}<(>) \frac{s_{i}}{s_{j}} \forall i, j$ if and only if $\psi_{j}<(>) \psi_{i}$. But if $\frac{p_{i}}{p_{j}}<(>) \frac{s_{i}}{s_{j}} \forall i, j$, then $s(\cdot)$ must be a monotonic OR (IR) of $p(\cdot)$ by Lemma 3 . Finally, the Envelope Theorem implies that $\Pi_{i}^{\prime}(x)=\left(P_{T}(T ; x) T^{\prime}(x)+P_{x}\right) p_{i} T$, where we know that $P_{T}(T ; x) d T+P_{x} d x>0$ from the proof of Proposition 8 . Thus $\Pi_{i}^{\prime}(x)>0$ 
$\forall i$, and $\Pi_{i}^{\prime}(x)-\Pi_{j}^{\prime}(x)=\left(P_{T}(T ; x) T^{\prime}(x)+P_{x}\right)\left(p_{i}-p_{j}\right) T>0$ whenever $j \triangleright i$.

Proof of Corollary 8 The first claim follows because, by Proposition 9, $s_{i}=p_{i} \forall i$ if and only if costs verify $\Phi(i, q)=c_{i} q^{\gamma}, \forall i \in I$, where the latter requirement again is equivalent to $x$ being inequality preserving by Proposition 8 . The second claim follows because, by Proposition 8. $d x>0$ induces an OR (IR) of $p(\cdot)$ whenever $\psi_{j}>(<) \psi_{i} \forall j \triangleright i$, and the latter condition is equivalent to $s(\cdot)$ being a monotonic OR (IR) of $p(\cdot)$ by Proposition 9.

Proof of Proposition 10 Note first that $P^{\prime}(\tau)<0$ and $T^{\prime}(\tau)>053$ Then, the equilibrium condition $P(\tau)+\tau=\varphi\left(i, p_{i} T\right)$ has the form of $(29)$, meaning that Propositions 89 and Corollary 8 apply. On the consumer-side, $P(\tau)=v\left(i_{c}, p_{i_{c}}^{d} T\right)$ shows that $\tau$ is a level variable with $T^{\prime}(\tau)>0$. Thus $\tau$ is inequality preserving if and only if $V\left(i_{c}, q\right)=\alpha_{i_{c}} q^{\mu} \forall i_{c}$ by Corollary 2, and the second claim follows from Corollary 4.

Proof of Proposition 11 The firm-side equilibrium condition (5) is $P=\varphi\left(i, p_{i} T\right)$, and hence identical to the one from Section 4.2.1. Aggregation yields $T=\int \varphi^{-1}(i, P) d i$, and $T^{\prime}(P)>0$ because $\varphi_{q}(i, q)>0 . T^{d}=T$ and aggregate demand imply that $P T(P)=\omega+\Pi(P)$, from which

$$
P^{\prime}(\omega)=\frac{1}{T(P)+P T^{\prime}(P)-\Pi^{\prime}(P)}
$$

follows. Hotelling's Lemma shows that $\Pi^{\prime}(P)=\int \partial_{P} \Pi_{i} d i=T$. Therefore $P^{\prime}(\omega)>0$, and $T^{\prime}(\omega)>0$, as well as $\Pi^{\prime}(\omega)=\Pi^{\prime}(P) P^{\prime}(\omega)>0$. Because $T^{\prime}(\omega)>0$ and $d \omega$ has the effect of a level variable for firms, the firm-side inequality must be as in Proposition 10 .

On the consumer-side, (31) implies that the ratio $p_{i_{c}}^{d} / p_{i_{c}}^{d}$ increases (decreases) in $\omega, j_{c} \triangleright i_{c}$, if

$$
\frac{d \omega+s_{i_{c}} \Pi^{\prime}(\omega)}{\omega_{i_{c}}+s_{i_{c}} \Pi}>(<) \frac{d \omega+s_{j_{c}} \Pi^{\prime}(\omega)}{\omega_{j_{c}}+s_{j_{c}} \Pi}, \quad \Pi^{\prime}(\omega) \equiv \Pi^{\prime}(P) P^{\prime}(\omega)
$$

(i) If $s_{i_{c}}=1 \forall i_{c}$, then $d \omega>0$ and $\Pi^{\prime}(\omega)>0$ imply by that the ratio $p_{i_{c}}^{d} / p_{j_{c}}^{d}$ decreases in $\omega$. Therefore $d \omega>0$ induces a monotonic IR of $p^{d}(\cdot)$ by Theorem 3 .

(ii) Using $\omega_{i_{c}}=\omega \forall i_{c}$ in 46 shows that $p_{i_{c}}^{d} / p_{j_{c}}^{d}$ increases (decreases) in $\omega$ if $\Pi^{\prime}(\omega)>\Pi(\omega) / \omega$. Because $\Pi^{\prime}(\omega)>0$ and $\Pi(0)=0$, it follows that $\Pi^{\prime}(\omega)>(<) \Pi(\omega) / \omega$ holds if $\Pi(\omega)$ is strictly convex (concave). Thus, if $\Pi(\omega)$ is strictly convex, $d \omega>0$ induces a monotonic OR.

Proof of Proposition 12 Note that $\alpha$ and $\theta$ are inequality preserving for firms. Equilibrium profits are $\Pi_{i}=p_{i} T \frac{\gamma-1}{\gamma}$, and thus aggregate profits are $\Pi \equiv \int \Pi_{i} d i=T \frac{\gamma-1}{\gamma}$. The equilibrium

\footnotetext{
${ }^{53}$ Formally, $T=\int \varphi^{-1}(i, P+\tau) d i \equiv T(P, \tau)$, and $T^{d}=\int v^{-1}\left(i_{c}, P\right) d i_{c} \equiv T^{d}(P)$, where $T_{P}(P, \tau)>0$, $T_{\tau}(P, \tau)>0$ and $T_{P}^{d}(P)<0$. Because $T=T(P, \tau)=T^{d}(P)=T^{d}$ we have $P^{\prime}(\tau)<0$, and thus $T^{\prime}(\tau)>0$.
} 
equation determining consumer market shares $\left(T^{D}=T\right)$ is $\alpha\left(w+S_{i_{c}}\right)=p_{i_{c}}^{d} T$; hence $T=$ $\alpha(w+\Pi)$ by summation. Substituting $\Pi$ yields

$$
T=\frac{\alpha \gamma}{\alpha+(1-\alpha) \gamma} w
$$

Further, integration of the firm-side optimality condition yields

$$
T=\left(\frac{\theta}{w \gamma}\right)^{\frac{1}{\gamma-1}} C, \quad C \equiv \int c_{i}^{\frac{1}{1-\gamma}} d i
$$

Equations 47), 480 imply that $w^{\prime}(\alpha)<0, T^{\prime}(\alpha)>0, w^{\prime}(\theta)>0$ and $T^{\prime}(\theta)>0$. Moreover, also $\frac{\partial \Pi_{i}}{\partial \alpha}>0$, and hence $\Pi^{\prime}(\alpha)>0$ and thus $\frac{\partial S_{i_{c}}}{\partial \alpha}>0$ (provided that $s_{i_{c}}>0$ ). Because aggregate output $T$ increases so must aggregate labor supply. To prove the claimed monotonic OR of $p_{i_{c}}^{d}$ for $d \alpha>0$, it suffices to show that $p_{i_{c}}^{d} / p_{j_{c}}^{d}$ increases in $\alpha$ by Theorem 3 . Note that $\frac{p_{i_{c}}^{d}}{p_{j_{c}}^{d}}=\frac{w+S_{i_{c}}}{w+S_{j_{c}}}$. Because $S_{i_{c}}=s_{i_{c}} \Pi$, capital incomes change in equal proportions, and because $p(\cdot)$ remains constant we obtain

$$
\frac{d S_{i_{c}}}{S_{i_{c}}}=\frac{d \Pi_{i}}{\Pi_{i}}=\frac{d T}{T}
$$

This together with the fact that $w^{\prime}(\alpha)<0$ and $\frac{\partial S_{i_{c}}}{\partial \alpha}>0$ implies that $\frac{p_{i_{c}}^{d}}{p_{j_{c}}^{d}}$ increases in $\alpha$, proving the OR of $p^{d}(\cdot)$ in $\alpha$. Moreover, because $q_{i_{c}}^{d}=p_{i_{c}}^{d} T$ this further assures also that $q_{i_{c}} / q_{j_{c}}$ and $f_{i_{c}} / f_{j_{c}}$ increase in $\alpha$. It now immediately follows that $d q_{i_{c}}>d q_{j_{c}}$ if $d q_{i_{c}}>0$. For $d \theta>0$, (47) and $\Pi=T \frac{\gamma-1}{\gamma}$ give $\frac{\Pi^{\prime}(\theta)}{\Pi(\theta)}=\frac{w^{\prime}(\theta)}{w(\theta)}$, which shows that relative income increases at the same rate for all consumers. Thus, $\theta$ must be inequality preserving.

Proof of Proposition 13 See main text.

Proof of Proposition 14 It is easy to see that $T^{\prime}(x)>0$ using the procedure from Section 3.3 , and the first claim follows from Corollary 2, With respect to $p(\cdot)$, the second claim follows from Theorem 3 noting that $p_{i} / p_{j}=\left(V_{i} / V_{j}\right)^{\frac{1}{\gamma-1}}$ As equilibrium profits are $\Pi_{i}=p_{i} V_{i} \frac{\gamma-1}{\gamma}$, it follows that $\frac{s_{i}}{s_{j}}$ is of the form (22), and the claimed inequality effects of $s(\cdot)$ follow from Proposition 4. The last claim follows from Lemma 3 as $s(i) / s(j)>p(i) / p(j)$.

Proof of Proposition 15 We prove the claim by applying Lemma 1. First, we note that $k_{m n}=1 \forall m, n \in[0,1]$. Second, we note that $R_{m n}=0$ whenever $m, n \neq i$, and $R_{i j}=\frac{d V_{i}}{V_{i}}>0$ whenever $j \triangleright i$, while $R_{j i}=-\frac{d V_{i}}{V_{i}}<0$ whenever $i \triangleright j$. These facts imply that $\Delta_{m}=\Delta_{n}$ whenever $m, n \neq i$. We now prove that $\Delta_{i}>0$ and $\Delta_{j}<0 \forall j \neq i$. By contradiction, suppose that $\Delta_{j} \geq 0$ for some $j \neq i$. Then actually $\Delta_{j}>0$ for all $j \neq i$. Because market shares must integrate to 
unity, it follows that $\Delta_{i}<0$, which is impossible because $\Delta_{i}=\Delta_{j}+R_{i j}>0$. We conclude that $\Delta_{i}>0$ while $\Delta_{j}<0 \forall j \neq i$, which further implies that only the market share of $i$ increases. The claimed proportionality in the decreasing market shares of the other agents follows directly from $\Delta_{m}=\Delta_{n}, m, n \neq i$.

Proof of Proposition 16 The equilibrium condition (5) is

$$
g(i) \equiv V_{1}+\left(1-2 p_{i}\right) V_{2}=c_{i} p_{i}^{\eta-1} T^{\eta} \equiv \varphi(i)
$$

By Proposition B.2 (Appendix B.4.2) the marginal change $d V_{1}, d V_{2}$ causes an OR (IR) of $p(\cdot)$ if

$$
z(p) \equiv \frac{d V_{1}+(1-2 p) d V_{2}}{V_{1}+(1-2 p) V_{2}}
$$

verifies $z^{\prime}(p)>(<) 0$. The first claim follows from $\operatorname{sign}\left(z^{\prime}(p)\right)=\operatorname{sign}\left(d V_{1} V_{2}-d V_{2} V_{1}\right)$. Likewise, Proposition B.2 (Appendix B.4.2 says that $R_{i j}=0$ uniformly if $z^{\prime}(p)=0$, which yields the second claim. The last claim follows from observing that $d w_{i}=2 d p_{i}\left(1-2 p_{i}\right)$, showing that if $p_{i}<1 / 2 \forall i$, then $w_{i}$ must behave like $p_{i}$ (as sign $\left.d w_{i}=\operatorname{sign} d p_{i}\right)$.

Proof of Proposition 17 The equilibrium equation is

$$
g(i) \equiv 2 \alpha p_{i} T+\beta=\gamma c_{i} p_{i}^{\gamma-1} T^{\gamma} \equiv \varphi(i)
$$

We first use the procedure in Section 3.3 to determine the sign of $T^{\prime}(\alpha)$. Implicit differentiation of $(50)$ for given $T>0$ shows that

$$
p_{T}(i ; T)=-\frac{g_{T}(i)-\varphi_{T}(i)}{g_{p}(i)-\varphi_{p}(i)}=-\frac{2 \alpha p_{i}(1-\gamma)-\beta \gamma / T}{2 \alpha T(2-\gamma)-\beta(\gamma-1) T / p_{i}}<0
$$

The same type of calculation shows that $p_{\alpha}(i ; T, \alpha)>0$, which allows us to conclude that $T^{\prime}(\alpha)>0$ by the procedure in Section 3.3 . To prove the claimed OR, we show that $R>0$ uniformly using $(10)$. Thus, we need to calculate the sign of $A(i)-A(j)$ for any $j \triangleright i$. As

$$
\frac{g_{\alpha}(i)}{g(i)}=\frac{2 p_{i} T}{2 \alpha p_{i} T+\beta}, \quad \frac{g_{T}(i)}{g(i)}=\frac{2 \alpha p_{i}}{2 \alpha p_{i} T+\beta}
$$

it is easily verified that $A(i)-A(j)>0$ proving that $R>0$ uniformly.

We now show that $R<0$ uniformly for $d \gamma>0$. We first show that $T^{\prime}(\gamma)<0$. We have

$$
p_{\gamma}(i ; T)=-\frac{g_{\gamma}(i)-\varphi_{\gamma}(i)}{g_{p}(i)-\varphi_{p}(i)}=\frac{c_{i} p_{i}^{\gamma-1} T^{\gamma}\left(1+\gamma L n\left(p_{i}\right)+\gamma L n(T)\right)}{2 \alpha T(2-\gamma)-\beta(\gamma-1) T / p_{i}}
$$


and thus $p_{\gamma}(i ; T)<0$ if $1+\gamma \operatorname{Ln}\left(p_{i}\right)+\gamma \operatorname{Ln}(T)<0$, where the last inequality holds as $p_{i}=t_{i} / T$ and $t_{i}>1$ by presumption. Together with $p_{T}(i ; T)<0$ this shows that $T^{\prime}(\gamma)<0$ by the procedure in Section 3.3 . To prove that $R<0$, we need to verify that $A(i)-A(j)<0$. But as $\frac{g_{\gamma}(i)}{g(i)}=1 / \gamma+\operatorname{Ln}\left(p_{i}\right)+\operatorname{Ln}(T)$, both $\frac{g_{\gamma}(i)}{g(i)}$ and $\frac{g_{T}(i)}{g(i)}$ are strictly increasing over agent types, $R<0$ follows from $T^{\prime}(\gamma)<0$, which proves the second claim. 


\section{B Supplement}

Section B.1 proves the equivalence between the equilibria where agents compete directly for market shares or indirectly via choosing their actions. Section B.2 establishes the existence of a unique equilibrium given Assumption 1 Section B.3 elaborates on the continuum representation for atomistic agents. Section B.4 contains additional technical results on rotations. Finally, Section B.5 demonstrates how to adopt the formal analysis to study inequality in the Nash equilibrium of aggregative games.

\section{B.1 Equivalence of equilibria}

We show that the model, where the agents optimize (2) by choosing $t(i)$ yields the same equilibria as the model, where agents optimize (4) by directly choosing their market shares. For simplicity, we refer to the model with payoff (2) as the "original model", and to the model with payoff (4) as the "transformed model". Recall that an equilibrium of the original model is given by $(t, T)$, where $t$ is the equilibrium action profile, such that $t(i)$ is the optimal action for each $i \in I$, and $T=Z(t)$. The following theorem shows that under the assumptions made, any equilibrium $(t, T)$ of the original model corresponds to an equilibrium $(p, T)$ of the transformed model, and vice-versa.

Theorem B.1 Suppose that (3) holds, and the market share function $p(i, \cdot, T)$ is bijective for any given $i \in I$ and any $T \in \mathbb{R}_{+}$.

1) If $(t, T)$ is an equilibrium of the original model and a function $p: I \rightarrow \mathbb{R}_{+}$is defined by $p(i)=p(i, t(i), T)$, then $(p, T)$ is an equilibrium in the transformed model.

2) If $(p, T)$ is an equilibrium of the transformed model and $t(i)=p^{-1}(i, p(i), T)$ for each $i \in I$, then $(t, T)$ is an equilibrium in the original model.

Proof: Recall the notational convention that we write $\Pi\left(i, p^{-1}(i, p(i), T), T\right) \equiv \Pi(i, p(i), T)$ for the payoff function where $t(i)$ has been replaced by the unique corresponding value $p(i)$, instead of using a separate symbolic notation $\Pi, \hat{\Pi}$ to explicitly distinguish between $\Pi(i, t(i), T)$ and $\hat{\Pi}(i, p(i), T)$. In particular, this means that $\Pi(i, t(i), T)=\Pi(i, p(i), T)$ whenever $p(i)=$ $p(i, t(i), T)$ or likewise $t(i)=p^{-1}(i, p(i), T)$.

1) If $(t, T)$ is an equilibrium, then $T=Z(t)$ and for each $i \in I, t(i)$ maximizes (2). Let $p(i) \equiv$ $p(i, t(i), T)$ denote the market shares induced by $(t, T)$. Suppose now that $(p, T)$ as constructed in the Theorem is not an equilibrium of the transformed model. Because $\int_{0}^{1} p(i) d i=1$ by (3), there then must be $\tilde{p}(i) \neq p(i)$ such that $\Pi(i, \tilde{p}(i), T)>\Pi(i, p(i), T)$ for some $i \in I$. Because 
$\tilde{t}(i)=p^{-1}(i, \tilde{p}(i), T) \neq t(i)=p^{-1}(i, p(i), T)$ this likewise implies that $\Pi(i, \tilde{t}(i), T)>\Pi(i, t(i), T)$ in the original model, contradicting the optimality of $t$ for all $i \in I$.

2) Let $(p, T)$ be an equilibrium of the transformed model, with market shares $p(i) \in \mathbb{R}_{+}$. Consider the action profile $t$ defined by $t(i)=p^{-1}(i, p(i), T), i \in I$. Because market shares $p(i)$ inversely are determined by $p(i)=p(i, t(i), T)$ and $1=\int_{0}^{1} p(i) d i=\int_{0}^{1} p(i, t(i), T) d i$, also $Z(t)=T$ by (3). Now, suppose that $(t, T)$ is not an equilibrium of the original model. Then, there is $\tilde{t}(i) \neq t(i)$ such that $\Pi(i, \tilde{t}(i), T)>\Pi(i, t(i), T)$ for some $i \in I$. Setting $\tilde{p}(i) \equiv p(i, \tilde{t}(i), T)$ for these $i \in I$ then implies that $\Pi(i, \tilde{p}(i), T)>\Pi(i, p(i), T)$, contradicting optimality of $p(i)$ in the transformed model.

\section{B.2 Equilibrium existence and uniqueness}

Assumptions 1 | 2 ascertain the existence of a unique equilibrium $(p(i), T)$.

Theorem B.2 (Existence and uniqueness) Any model with payoffs (4) that satisfy Assumptions 1 and 2 has a unique equilibrium $(p(i), T)$. All equilibrium payoffs $\Pi(i)$ are positive, and $p(\cdot)$ is a bounded, decreasing and strictly positive density.

The proof evolves in two steps, reflecting the two requirements in the equilibrium definition. Its baseline reasoning is illustrated in Figure 3. (A1) implies that a unique optimizer $p(i ; T)>0$
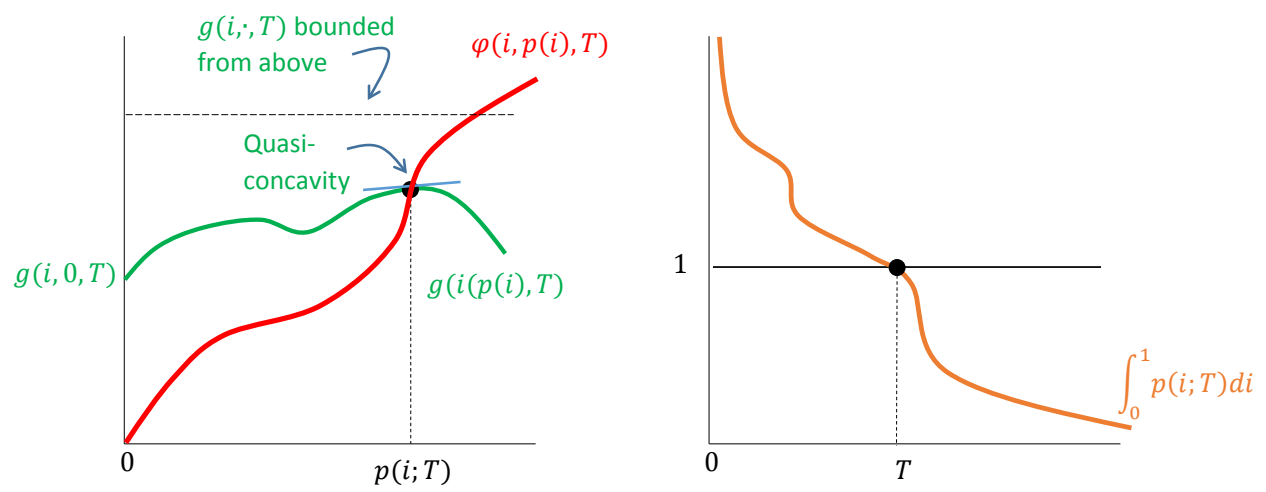

Figure 3: Equilibrium existence and uniqueness

exists for any given $T>0$ and any fixed $i \in[0,1]$. This follows because a zero market share is not optimal $(g(i, 0, T)>0=\varphi(i, 0, T))$, the gains from increasing one's market share are limited $(g(\cdot, T)$ bounded from above) and marginal costs are strictly increasing in $p(i)$ and unbounded. Uniqueness of this optimizer is implied by strong quasiconcavity. (A2) then assures that there is a unique $T>0$ such that $\int p(i ; T) d i=1$. To see why, suppose that $g(i, p, T)$ is bounded above and away from 0 for any $p \geq 0$ and any $T>0$, consistent with (but stronger than) assumption (A2). Even the best agent $(i=0)$ then seeks to make her market share $p(i ; T)$ arbitrarily small 
because as $T \rightarrow \infty$ marginal costs become arbitrarily large. Similarly, even the worst agent $(i=1)$ aims at an arbitrarily large $p(i ; T)$ if $T \rightarrow 0$ and marginal costs become arbitrarily small. These two facts imply that $\lim _{T \rightarrow \infty} \int p(i ; T) d i=0$ and $\lim _{T \rightarrow 0} \int p(i ; T) d i=\infty$, and existence of a $T>0$ with $\int p(i, T) d i=1$ follows by continuity of $\int p(i ; \cdot) d i$. Uniqueness then follows from the last assumption in (A2), which assures that $\int p(i ; \cdot) d i$ is strictly decreasing at $\int p(i, T) d i=1$.

Proof of Theorem B.2 The proof consists of two steps. i) Fix $i \in[0,1]$ and $T>0$ arbitrarily. (A1) assures that the equation (5) has a unique solution $p(i ; T)>0$, and that this solution indeed maximizes (4) given $T$. Now, consider the function $p(i, T) \equiv p(i ; T)$, noting that $p(i, \cdot)$ is a strictly decreasing $C^{1}$-function on $(0, \infty)$ as a consequence of the Implicit Function Theorem, the strong quasiconcavity assumption in (A1), and the last assumption of (A1). Moreover, $p(\cdot, T)$ must be a decreasing function by Assumption 2 and, hence, $p(\cdot, T)$ is integrable over $[0,1]$, so let $G(T) \equiv \int_{0}^{1} p(i, T) d i$, noting that $G$ is differentiable. ii) We show: $\exists ! T>0$ : $G(T)=1$. Fix $i \in[0,1]$. By (A2) there must exist $T_{i}>0: g\left(i, 1, T_{i}\right)=\varphi\left(i, 1, T_{i}\right)$. Therefore, $\exists T_{0}>0$ such that $p\left(0, T_{0}\right)=1$. Because $p(i, \cdot)$ strictly decreasing, it follows that $p(0 ; T)<1$ for $T>T_{0}$. Since $p(\cdot, T)$ is decreasing, we must have $p(i, T)<1$ for any $i \in[0,1]$ and $T>T_{0}$, which implies that $\lim _{T \rightarrow \infty} G(T)<1$. Similarly, it follows that $\exists T_{1}>0$ such that $p\left(1 ; T_{1}\right)=1$. Thus $p\left(i, T_{1}\right)>1$ for $i \in[0,1]$ and $T<T_{1}$, hence $\lim _{T \rightarrow 0} G(T)>1$. As $G(\cdot)$ continuous, $\exists T>0$ such that $G(T)=1$, and uniqueness follows from the fact that, for each $i \in[0,1], p(i ; T)$ and hence $G(T)$ is strictly decreasing in $T$. Finally, $\Pi(i)>0$, because $p(i)=p(i ; T)>0$ is the unique maximizer and $\left.\Pi(i)\right|_{p(i)=0}=0$.

\section{B.3 Continuum representation for atomistic agents}

We illustrate that the equilibrium distribution in case of $n \in \mathbb{N}$ atomistic ("discrete") agents can be identified with our finite step density model. The following argument considers the case, where heterogeneity enters the model through a cost coefficient function as in (6). This should suffice to make evident that the representation result applies similarly to other cases as well. Consider a population consisting of $n \in \mathbb{N}$ atomistic (or "discrete") agents, indexed by $\{1 / n, 2 / n, \ldots, 1\}$. Suppose that the agents differ in their cost coefficient $c(i), i \in\{1 / n, 2 / n, \ldots, 1\}$. Then, the agents can be partitioned into $1 \leq K \leq n$ groups of identical agents, with group size $n_{k}, \sum_{k} n_{k}=n$. This partition gives $1 \leq K \leq n$ equivalence classes (groups) of sizes $n_{1}, \ldots, n_{K}$, $\sum_{k} n_{k}=n$. We identify each group by a "representative" agent $i_{k}$. In equilibrium every agent $(i / n)$ chooses $p^{d}(i / n)$ ( $d$ for "discrete") to maximize her payoff $\Pi(i)$, where $p^{d}(i / n)$ must satisfy $\sum_{i=1}^{n} p^{d}(i / n)=1$. Let $p(i)$ denote the (step) density function that characterizes our (continuum) equilibrium from definition 1 with the corresponding cost step function $c(i)=c\left(i_{k}\right)$ 
on $\left[i_{k}, i_{k+1}\right)$, and group measures $\gamma_{1}, \ldots, \gamma_{K}$ satisfying $\gamma_{k}=n_{k} / n$. We now establish the formal equivalence between the discrete equilibrium probability distribution $\left\{p^{d}(1 / n), \ldots, p^{d}(1)\right\}$ and the equilibrium step density $p(i)$.

Theorem B.3 (Continuum Representation) Let $n \in \mathbb{N}$ and suppose that agents are partitioned in $K$ cost groups. If $\left\{p^{d}(i / n)\right\}$ corresponds to the discrete equilibrium and $p(i)$ is the equilibrium (step) density of the respective continuum problem, then $p^{d}(i / n)=\frac{1}{n} p(i / n)$ is satisfied for all $i \in\{1, \ldots, n\}$

Proof: In the continuum case we only have to solve the optimization problem for a representative agents $i_{k}$. In the discrete equilibrium $1=\sum_{i=1}^{n} p^{d}(i / n)=\sum_{k=1}^{K} p^{d}\left(i_{k}\right) n_{k}$. The claim now is that $\frac{1}{n} p\left(i_{k}\right)=p^{d}\left(i_{k}\right)$ for $k=1, \ldots, K$. But because in the continuum equilibrium we must have

$$
1=\int_{0}^{1} p(i) d i=\sum_{k=1}^{K} p\left(i_{k}\right) \gamma_{k}=\sum_{k=1}^{K}\left(\frac{1}{n} p\left(i_{k}\right)\right) n_{k}
$$

the claim follows from the uniqueness of equilibrium.

Hence the continuum step-function case and the atomistic case are equivalent up to the multiplicative constant $1 / n$ (independent of group composition), which means that we can work with either model, and justifies our procedure of the main text. It then also follows that $p\left(i_{k}\right) \gamma_{k}=p^{d}\left(i_{k}\right) n_{k}$ corresponds to the market share of a member of group $k$, illustrating why we used the notion of a "representative" agent.

Because Theorem B.3 remains valid as $n$ grows arbitrarily large, this provides the following justification for using strictly increasing cost coefficient functions (Class II) as an approximation for the case of many different agents. Suppose that $c(i)$ is a Class II function defined on $[0,1]$ (e.g., $c(i)=1+i$ ), and let $p(i)=p(c(i))$ denote the corresponding equilibrium density (a strictly decreasing, continuous function). Then, because $c(i)$ is continuous on a compact interval, for $n \in \mathbb{N}$ the sequence of step functions defined by $c_{n}(i)=c(i)$ if $i \in\{0,1 / n, 2 / n, \ldots, 1\}$ and $c_{n}(i)=$ $c(s / n)$ for $i \in(s / n,(s+1) / n), s \in\{0,1, \ldots, n-1\}$, converges (uniformly) to $c(i)$ as $n \rightarrow \infty 54$ Consider the atomistic equilibrium distribution $p^{d}(i / n)$ induced by $c(0), c(1 / n), c(2 / n), \ldots, c(1)$. By Theorem B.3, $n p^{d}(i / n)=p(i / n)$, where $p(i / n)$ is the step-density version of $p^{d}(i / n)$. More precisely, for a given $n \in \mathbb{N}$ this density is a decreasing finite step function with $p_{n}(i)=p\left(c_{n}(i)\right)$, where $c_{n}(i)$ is as defined above. Because $c_{n}(i) \rightarrow c(i)$ and $p(i)$ is continuous, we have $p(i)=$ $p(c(i))=p\left(\lim c_{n}(i)\right)=\lim p\left(c_{n}(i)\right)=\lim p_{n}(i)$. This shows that while, of course, the atomistic $p^{d}(i / n)$ becomes arbitrarily close to zero as $n$ grows large, the "scaled" distribution law as captured by the step-density version $p(i / n)$ approaches $p(i)$.

\footnotetext{
${ }^{54}$ Such approximations of continuous functions by a sequence of step functions are a standard result in real analysis and integration theory.
} 


\section{B.4 Rotations: Further Results}

\section{B.4.1 Ratio Test}

We prove that condition 15 from Section 3.2 .2 indeed implies a rotation of $p(\cdot)$.

Proposition B.1 Suppose that $\infty>p\left(\cdot ; x^{\prime}\right), p(\cdot ; x)>0$ are right-continuous, decreasing SSD densities with similar equivalence classes $[i]$. If 15$)$ holds, then $p\left(\cdot ; x^{\prime}\right)$ is an OR (IR) of $p(\cdot ; x)$.

Proof: Let $g(i) \equiv \frac{p\left(i ; x^{\prime}\right)}{p(i ; x)}$. Because $p\left(\cdot ; x^{\prime}\right)$ and $p(\cdot ; x)$ both are SSD densities with similar equivalence classes, 15$)$ implies that $g(\cdot)$ is not constant on $(0,1): \exists i_{0} \in(0,1)$ such that $g(i)>$ $g(j), i<i_{0} \leq j$. Further, $g(\cdot)$ is right-continuous, and by (15) also decreasing. We first claim that $g(0)>1$. Suppose, by contradiction, that $g(0) \leq 1$. Then $g(i) \leq 1, i \in I$, and additionally $g(j)<1$ for $j \geq i_{0}$. This implies that $p\left(i ; x^{\prime}\right) \leq p(i ; x), i \in I$, and $p\left(j ; x^{\prime}\right)<p(j ; x)$ for $j \geq i_{0}$. Hence $\int p\left(s ; x^{\prime}\right) d s<\int p(s ; x) d s$, contradiction. Therefore $g(0)>1$; a similar argument shows that $g(1)<1$. Because $g(0)>1$ and $g(\cdot)$ is right-continuous, the set $\{i: g(i)>1, i>0\}$ is non-empty, and we let $i_{0} \equiv \sup \{i>0: g(i)>1\}$, noting that $i_{0} \in(0,1)$. Because $g$ decreases and $g(1)<1$, the set $\left\{i: g(i)<1, i \geq i_{0}\right\}$ is non-empty, and we set $i_{1} \equiv \inf \left\{i \geq i_{0}: g(i)<1\right\}$. If $i_{0}=i_{1}$, then $p\left(i ; x^{\prime}\right)>p(i ; x)$ on $\left(0, i_{0}\right)$, and $p\left(i ; x^{\prime}\right)<p(i ; x)>0$ on $\left(i_{0}, 1\right]$. If $i_{0}<i_{1}$ then $g(i)=1$ on $\left(i_{0}, i_{1}\right)$. These facts together imply that $p\left(\cdot ; x^{\prime}\right)$ is OR of $p(\cdot ; x)$; the case on an IR is proved similarly.

\section{B.4.2 On the Direct-Aggregative Effect}

By Theorems 1 and 2, the sign of the direct-aggregative effect is crucial for studying the inequality effects. In this section we search for the determinants of $\operatorname{sign}\left(R_{i j}\right)$ in terms of primitives. We concentrate on the multiplicatively separate case where $\varphi(i)=\varphi(i, p) C(T)$ as many of our applications feature such a cost function.

Proposition B.2 Let $\varphi(i)=\varphi(i, p) C(T)$, and define $h(i, p, T ; x)=\operatorname{Ln}(g(i, p, T ; x))$. If

$$
\begin{array}{ll}
h_{x}\left(i, p, T ; x_{0}\right) \geq h_{x}\left(j, p, T ; x_{0}\right), \quad h_{T}\left(i, p, T ; x_{0}\right) \geq h_{T}\left(j, p, T ; x_{0}\right) \quad \forall j \triangleright i, \\
h_{T}\left(i, p^{\prime}, T ; x_{0}\right) \geq h_{T}\left(i, p, T ; x_{0}\right), \quad h_{x}\left(i, p^{\prime}, T ; x_{0}\right) \geq h_{x}\left(i, p, T ; x_{0}\right) \quad \forall i \text { and any } p^{\prime}>p,
\end{array}
$$

where at least one of the above inequalities is strict, then $R$ is uniformly positive at $x_{0}$. If all inequalities are reversed (and one strict so), then $R$ is uniformly negative at $x_{0}$. Finally, if all inequalities are equalities, then $R$ is uniformly zero at $x_{0}$. 
Proof: We only prove the uniformly positive case (the negative case is established by the same type of arguments). We need to show that for

$$
A(i)=\frac{g_{T}(i)}{g(i)} T^{\prime}(x)+\frac{g_{x}(i)}{g(i)}
$$

we have $A(i)>A(j)$ whenever $j \triangleright i$. So take any $j \triangleright i$. First, $h_{T}\left(i, p^{\prime}, T ; x_{0}\right) \geq h_{T}\left(i, p, T ; x_{0}\right)$ and $h_{T}\left(i, p, T ; x_{0}\right) \geq h_{T}\left(j, p, T ; x_{0}\right)$ yield

$$
h_{T}\left(i, p(i), T ; x_{0}\right) \geq h_{T}\left(i, p(j), T ; x_{0}\right) \geq h_{T}\left(j, p(j), T ; x_{0}\right)
$$

and because $T^{\prime}(x)>0$ (see Lemma 4, Section 3.3 and $h_{T}=\frac{g_{T}}{g}$ we have

$$
\frac{g_{T}(i)}{g(i)} T^{\prime}(x) \geq \frac{g_{T}(j)}{g(j)} T^{\prime}(x)
$$

where the inequality is strict, whenever at least one of the initial inequalities is strict. Second, $h_{x}\left(i, p, T ; x_{0}\right) \geq h_{x}\left(j, p, T ; x_{0}\right)$ and $h_{x}\left(i, p^{\prime}, T ; x_{0}\right) \geq h_{x}\left(i, p, T ; x_{0}\right)$ yield

$$
h_{x}\left(j, p(j), T ; x_{0}\right) \leq h_{x}\left(j, p(i), T ; x_{0}\right) \leq h_{x}\left(i, p(i), T ; x_{0}\right)
$$

and hence also

$$
\frac{g_{x}(i)}{g(i)} \geq \frac{g_{x}(j)}{g(j)}
$$

where, again, the inequality is strict if one of the previous inequalities is strict. This shows that $R_{i j}\left(x_{0}\right)>0$. Finally, is all inequalities are equalities, the condition in Proposition B.2 is equivalent to multiplicative separability of $g(i)$ in $(i, p)$ and $(T, x)$, meaning that $x$ must be a level variable, and the last claim follows from Corollary 1.

Proposition B.2 contains the inequality analysis of the contest model from section 4.3 as the special case, where marginal benefits in (5) are independent from $p$ and $T$. In such a case, Proposition B.2 tells us that only the direct effect of $d x$ matters for the inequality effects. In particular, incentives to increase market shares are relatively stronger for strong agent types if and only if marginal benefits increase proportionally more for these types, i.e., iff $d g(i) / g(i)>$ $d g(j) / g(j)$ holds for $j \triangleright i$. In the more general case, where marginal benefits depend also on $p$ and $T$, the above effect is mitigated. For example, a positive shock $d x>0$ increases $T$ by Lemma 4, and the direct incentive effects of $d x>0$ are either reinforced or weakened in response 
to $d T$ depending on $g_{T} 55$

As another application, suppose that marginal benefits can be written as a power function of the form

$$
g(i, p, T, x)=u(i) v(T ; x) p^{\xi(T ; x)}
$$

Proposition B.2 then implies that $R$ is determined solely by the elasticity function $\xi(T, x)$.

In particular, $R$ is uniformly positive if $\xi_{T}, \xi_{x} \geq(\leq) 0$ with one strict inequality, and $R=0$ uniformly if $\xi$ is constant.

\section{B.4.3 Equilibrium CDF}

The following proposition describes the $\operatorname{CDF} F(i ; x)$ pertaining to the equilibrium market share function $p(i ; x)$ in greater detail, and clarifies the effects of a rotation on the ordering of the CDF's.

Proposition B.3 Suppose that $\forall x \in X$ the decreasing density $p(\cdot ; x)$ with support $[0,1]$ and $C D F F(\cdot ; x)$ verifies the SSD property. Then

a) $F(i ; x)>i, i \in(0,1), x \in X$

b) $F(i ; x)$ is strictly increasing, continuous and concave in $i, x \in X$. If $p(\cdot ; x)$ is strictly decreasing, then $F(\cdot ; x)$ is strictly concave.

c) If $p\left(i ; x^{\prime}\right)$ is OR of $p(i ; x)$, then $F(\cdot ; x)$ strictly stochastically dominates $F\left(\cdot ; x^{\prime}\right)$, i.e. $F\left(i ; x^{\prime}\right)>F(i ; x), \forall i \in(0,1)$.

Proof a) Fix $x \in X$; hence we omit to write $x$ in the proof of a). Consider $i_{0} \in(0,1)$. If $p\left(i_{0}\right) \geq 1$, then by SSD and because $p(\cdot)$ is a decreasing density $\exists \hat{i}<1: p(i)<1$ on $[\hat{i}, 1]$. Thus there must also be $\tilde{i} \in\left(0, i_{0}\right]$ such that $p(i)>1$ on $[0, \tilde{i})$. Hence $F\left(i_{0}\right)>i_{0}$. If $p\left(i_{0}\right)<1$ then, by SSD and the decreasing density property, $\exists i \in\left(0, i_{0}\right]$ :

$$
F\left(i_{0}\right)=\int_{0}^{i} \underbrace{p(s)}_{\geq 1} d s+\int_{i}^{i_{0}} \underbrace{p(s)}_{<1} d s
$$

Then $\int_{0}^{i}(p(s)-1) d s=\int_{i}^{1}(1-p(s)) d s>\int_{i}^{i_{0}}(1-p(s)) d s$, where the inequality follows from the fact that $1-p(s)>0$ for $s \in[i, 1]$. Hence $F\left(i_{0}\right)>i_{0}$.

\footnotetext{
${ }^{55}$ Particularly, if $g_{T}>(<) 0$, then $R>0$ is more likely to result if the increase (decrease) in marginal benefits triggered by $d T>0$ affects the strong agent relatively more (less).
} 
We now prove c), which follows from a similar argument. If $i \in\left(0, i_{0}\right]$, then $p\left(i ; x^{\prime}\right)>p(i ; x)>$ 0 for $i \in\left(0, i_{0}\right)$, and hence $F\left(i ; x^{\prime}\right)>F(i ; x)$. If $i \in\left(i_{0}, 1\right)$, then

$$
\int_{0}^{i_{0}}\left(p\left(s ; x^{\prime}\right)-p(s ; x)\right) d s=\int_{i_{0}}^{1}\left(p(s ; x)-p\left(s ; x^{\prime}\right)\right) d s>\int_{i_{0}}^{i}\left(p(s ; x)-p\left(s ; x^{\prime}\right)\right) d s
$$

where the inequality follows from $p\left(s ; x^{\prime}\right)=p(s ; x)$ on $\left(i_{0}, i_{1}\right)$ and $p(s ; x)>p\left(s ; x^{\prime}\right)$ on $\left(i_{1}, 1\right]$. Hence again $F\left(i ; x^{\prime}\right)>F(i ; x)$.

b) As in the proof of a) we fix $x \in X$ and omit to write $x$ in the following. The fact that $F(i)$ is strictly increasing in $i$ follows directly from $p(\cdot)>0$. Let $i, j \in[0,1]$ and $i<j$. Let $t \in[0,1]$. First, note that $t F(i)+(1-t) F(j)=F(j)-t \int_{i}^{j} p(s) d s$, and $F(t i+(1-t) j)=$ $\int_{0}^{j} p(s) d s-\int_{i^{\prime}}^{j} p(s) d s$ where $i^{\prime}=t i+(1-t) j$. From these two expressions it follows that $F$ is concave iff $t \int_{i}^{j} p(s) d s \geq \int_{i^{\prime}}^{j} p(s) d s$, or equivalently if

$$
t \int_{i}^{i^{\prime}} p(s) d s-(1-t) \int_{i^{\prime}}^{j} p(s) d s \geq 0 \quad t \in(0,1), i<i^{\prime}<j
$$

Because $p(i)$ is decreasing, we must have $p(i) \geq p\left(i^{\prime}\right) \geq p(j)$ (with strict inequalities in the strictly decreasing case). Hence (51) is satisfied (strictly so in the strictly decreasing case) if

$$
t \int_{i}^{i^{\prime}} p\left(i^{\prime}\right) d s-(1-t) \int_{i^{\prime}}^{j} p\left(i^{\prime}\right) d s \geq 0 \quad t \in(0,1)
$$

This inequality reduces to $t\left(i^{\prime}-i\right)-(1-t)\left(j-i^{\prime}\right) \geq 0$, which, by construction of $i^{\prime}$, is satisfied. This verifies the (strict) concavity of $F(\cdot)$, and the continuity of $F(\cdot)$ follows from concavity and the fact that $F(\cdot)$ cannot jump at the boundary points $\{0,1\}$. 


\section{B.4.4 Difference Test}

We next provide an alternative sufficient condition to detect rotations. If $p\left(\cdot ; x^{\prime}\right)-p(\cdot ; x)$ is strictly decreasing over its equivalence classes, i.e., if $p\left(\cdot ; x^{\prime}\right)-p(\cdot ; x)$ is strictly decreasing for any two different agent types, then $p\left(\cdot ; x^{\prime}\right)$ must be an OR of $p(\cdot ; x)$.

Proposition B.4 (Difference test) Take the presumptions of Proposition B.1 as satisfied. If

$$
p\left(i ; x^{\prime}\right)-p(i ; x)>(<) p\left(j ; x^{\prime}\right)-p(j ; x) \quad \text { whenever } j \triangleright i \in(0,1)
$$

holds, then $p\left(\cdot ; x^{\prime}\right)$ is an $O R(I R)$ of $p(\cdot ; x)$.

Proof The proof mimics the one of Proposition B.1. Define $g(i) \equiv p\left(i ; x^{\prime}\right)-p(i ; x)$, and note that $\int g(s) d s=0$. Suppose that $g(0) \leq 0$ By presupposition, $g$ is decreasing, right-continuous and, by SSD, $\exists i_{0} \in(0,1): 0 \geq g(0)>g(i), \forall i \geq i_{0}$. Hence $\int g(s) d s<0$, a contradiction. Therefore $g(0)>0$, and a similar argument shows that $g(1)<0$. Because $g$ is decreasing, right-continuous and $g(0)>0$, the set $\{i: g(i)>0, i>0\}$ is non-empty, and we let $i_{0}=\sup \{i>0: g(i)>0\}$, noting that $i_{0} \in(0,1)$. It follows that $p\left(i ; x^{\prime}\right)>p(i ; x)$ on $\left(0, i_{0}\right)$, and $\int_{0}^{i_{0}} g(s) d s>0$. Because $g$ decreases and $\int g(s) d s=0$, the set $\left\{i: g(i)<0, i \geq i_{0}\right\}$ is non-empty, and we set $i_{1}=\inf \left\{i \geq i_{0}: g(i)<0\right\}$. If $i_{0}<i_{1}$ then $g(i)=0$ on $\left(i_{0}, i_{1}\right)$, as $g$ is decreasing and rightcontinuous. These facts together imply that $p\left(\cdot ; x^{\prime}\right)$ is OR of $p(\cdot ; x)$.

Like Proposition B.1, Proposition B.4 encompasses both Class I and II densities. The counterpart of Theorem 3 is as follows ${ }^{56}$

Corollary B.1 Suppose that the presumptions of Theorem 3 hold. Let $x_{0} \in X$. If

$$
\frac{\partial}{\partial x}\left(p\left(i ; x^{\prime}\right)-p\left(j ; x^{\prime}\right)\right)>0 \quad \forall x^{\prime} \geq x_{0}
$$

$\forall j \triangleright i$, then $p\left(i ; x^{\prime}\right)$ is OR of $p(i ; x)$ whenever $x^{\prime}>x_{0}$. If the inequality in (16] is reversed, then $p\left(i ; x^{\prime}\right)$ is IR of $p(i ; x)$.

For applications, Corollary B.1 says that whenever we can deduce 53 from the equilibrium equation (7), then the OR property follows.

Relation to submodularity If $p(\cdot ; \cdot)$ is strictly submodular (or log-submodular), then (52) (or (15)) is satisfied with " $>$ ", but if $p(\cdot ; x)$ is a Class I step-function, $p(\cdot ; \cdot)$ is never strictly

\footnotetext{
${ }^{56}$ The proof is similar to the one of Theorem 3 and therefore omitted.
} 
submodular, because strict submodularity of $p(\cdot ; \cdot)$ requires $p\left(\cdot ; x^{\prime}\right)-p(\cdot ; x)$ to be strictly decreasing for $x^{\prime}>x$, which obviously is violated in the step-function case (a similar argument holds for the log-submodular case) ${ }^{57}$ This further shows that in case of Class II densities if $(52)$ holds for all $x, x^{\prime} \in X$ with $x^{\prime}>x$ then $p(\cdot ; \cdot)$ is strictly submodular as a function. A similar argument can be made in the supermodular case.

Relation between difference and ratio test We now discuss the formal relation between the difference and the ratio test, and relate it to the literature. We begin by noting that in the two-type case properties (15), (52), the rotation-property (12) and stochastic dominance of the respective distribution functions are all equivalent (we omit the obvious proof). In the case of just two types, suppose that the fraction of "good" (e.g., low-cost) types is $\gamma \in(0,1)$. Let $i=0$ represent good types and $i=1$ bad types. Then $p(\cdot)$ has the form

$$
p(\cdot)=\left\{\begin{array}{ll}
p_{0} & i \in[0, \gamma) \\
p_{1} & i \in[\gamma, 1]
\end{array} \quad, p_{1}=\frac{1-\gamma p_{0}}{1-\gamma} \quad, p_{0} \geq p_{1}\right.
$$

Proposition B.5 (Two-types case) Let $x, x^{\prime} \in X$ and suppose that the densities $p(\cdot ; x), p\left(\cdot ; x^{\prime}\right)$ are specified by (54) with distribution functions $F(\cdot ; x), F\left(\cdot ; x^{\prime}\right)$. Then properties (12), 15), (52) and strict stochastic dominance $F\left(i ; x^{\prime}\right)>F(i ; x)$ are equivalent.

While the ratio test and the difference test can both be used to establish an OR or IR of $p(\cdot)$, they are not equivalent in general 58

Proposition B.6 Suppose that the premise of Proposition B.1 is satisfied. If (15) is satisfied, then (52) holds for all $i$ for which $p\left(i ; x^{\prime}\right) \geq p(i ; x)$. If (52) is satisfied, then (15) holds for all $j \triangleright i$ where $p\left(i ; x^{\prime}\right) \leq p(i ; x)$.

Proof: If 15 is satisfied, then equivalently $\frac{p\left(i ; x^{\prime}\right)-p(i ; x)}{p(i ; x)}>\frac{p\left(j ; x^{\prime}\right)-p(j ; x)}{p(j ; x)}$ whenever $j \triangleright i$. Suppose that $p\left(i ; x^{\prime}\right) \geq p(i ; x)$, and take $j \triangleright i$. Hence $p(i ; x)>p(j ; x)$, and the first claim follows from $\frac{p\left(i ; x^{\prime}\right)-p(i ; x)}{p(j ; x)}>\frac{p\left(j ; x^{\prime}\right)-p(j ; x)}{p(j ; x)}$. If 52 is satisfied, then

$$
\frac{p\left(i ; x^{\prime}\right)}{p(i ; x)}>\frac{p\left(j ; x^{\prime}\right)-p(j ; x)}{p(i ; x)}+1 \quad j \triangleright i
$$

\footnotetext{
${ }^{57}$ Recall that for functions on $\mathbb{R}^{2}$ strict super- and submodularity are equivalent to the concept of strictly increasing (decreasing) differences.

${ }^{58}$ The ratio condition $(15)$ and the difference condition $(52)$ are equivalent if $p(i ; x), p\left(i ; x^{\prime}\right)$ are linear in $i$, which they cannot be if $p(\cdot)$ is of Class I.
} 
Now, because of (55) condition (15) is satisfied if $\frac{p\left(j ; x^{\prime}\right)-p(j ; x)}{p(i ; x)}+1 \geq \frac{p\left(j ; x^{\prime}\right)}{p(j ; x)}$, or equivalently, if $\frac{p\left(j ; x^{\prime}\right)-p(j ; x)}{p(i ; x)} \geq \frac{p\left(j ; x^{\prime}\right)-p(j ; x)}{p(j ; x)}$. But this inequality must hold, because as $p\left(i ; x^{\prime}\right) \leq p(i ; x)$ 55 implies that $p\left(j ; x^{\prime}\right)-p(j ; x)<0$.

Proposition B.6 says that if $p$ satisfies 52 , then the ratio $p(i ; x) / p(j ; x)$ increases in $x$ on the "loosing side" where $p\left(i ; x^{\prime}\right) \leq p(i ; x)$. Conversely, if the ratio property (15) is satisfied, then the "winning side", where $p\left(i ; x^{\prime}\right) \geq p(i ; x)$, satisfies property (52).

To place this result in the relevant mathematical context, note that if $p(\cdot ; \cdot)$ were monotonic on $[0,1] \times X$, then log-supermodularity of $p(\cdot ; \cdot)$ would imply supermodularity, and submodularity would imply log-submodularity (see Topkis (1998), p. 65). However, because in our context $p(i, x)$ generically cannot be montonic in both arguments ${ }^{59}$ this result does not apply. In this respect, Proposition B.6 can be viewed as an extension of the result to the case of a partially monotone function.

\section{B.5 Market Inequality in the Nash Equilibria of Aggregative Games}

The aim of this section is to exemplify, by means of a simple application from contest theory, that the approach of this paper can be applied to study inequality effects in aggregative games with payoffs of the form (2). The only essential difference to the setting of the main text is that in an aggregative game the individual agents take into account the effects of their own actions on the aggregate in a Nash equilibrium. If the aggregate $T$ in payoff (2) is not exogenous to the individual player, it is not obvious whether and how our distributional tools may be used to study the inequality effects.

We now exemplify how our approach can be adjusted to be fruitful also in this more cumbersome case. The way how we proceed to use our inequality tools if individual players take into account their impact on the aggregate is general, and can be used in any other aggregative game with a differentiable structure as well. Note that there is little loss in assuming a sum-aggregative structure of such games, because any aggregative game with a well-behaved aggregator (differentiable and coordinate-wise strictly monotonic) is strategically equivalent to a sum-aggregative game (Cornes and Hartley, 2012).

In Section 4.3 we analyzed a contest model under the assumption that individual agents take the aggregate $T=\int t(i) d i$ as given when choosing their effort, while $T$ was endogenous to the model. We now study the same model, assuming that each of finitely many agent types takes its

\footnotetext{
${ }^{59}$ E.g. in the continuous case the integral condition implies the existence of at least one intersection of $p(i ; x)$ and $p\left(i ; x^{\prime}\right)$.
} 
own effect on the aggregate into consideration. Consider a fixed-prize contest with $n$ atomistic agents and payoffs

$$
\Pi_{i}=\frac{t_{i}}{\sum t_{s}} V-\Phi\left(i, t_{i}\right)
$$

Define the market share $p_{i}=t_{i} / T$ as before, with $T \equiv \sum t_{s}$. Let $T_{i} \equiv \sum_{s \neq i} t_{s}$. Because $T=T_{i}+t_{i}$ and $t_{i}=p_{i} T$ we obtain $t(i)=\frac{p_{i}}{1-p_{i}} T_{i}$. Thus we can restate (56) in terms of own market share as

$$
\Pi_{i}=p_{i} V-\Phi\left(i, \frac{p_{i}}{1-p_{i}} T_{i}\right)
$$

where $T_{i}$ is exogenous to player $i$. A Nash equilibrium is a probability vector $\left(p_{1}, \ldots, p_{n}\right)$ and an aggregate $T>0$ such that $T_{i}=\left(1-p_{i}\right) T$ and $p(i)$ maximizes $\left.(56]\right){ }^{60}$ Thus, any interior Nash equilibrium satisfies the FOC system

$$
V=\frac{\varphi\left(i, p_{i} T\right)}{1-p_{i}} T
$$

Because (57) is of the form (5), we can apply the inequality tools from Section 3 as they are to study the inequality effects as we did in the main text 61

One question of self-interest is whether the inequality predictions are sensitive to the change in the equilibrium concept. For example, Acemoglu and Jensen (2010) find that sometimes individual strategies may respond differently to exogenous shocks if players take their impact on the aggregate into account. In this respect, the following proposition shows that we find the same inequality effects induced by an increase in the common prize value $d V>0$ in case of Nash equilibria, at least in the present contest model.

Proposition B.7 The inequality effects induced by $d V$ are determined by the t-elasticity of marginal costs alone. If $\Phi(i, t)=c_{i} t^{\gamma}$, then $p(\cdot)$ is invariant to $x$. If $\Phi(i, t)=c^{\gamma_{i}}$ such that $p_{i}$ is of Class I, then $d V>0$ causes an OR of $p(\cdot)$.

Proof: Evaluating (10) in case of (57) and using $t_{i}=p_{i} T$ yields

$$
\operatorname{sign}\left(R_{i j}\right)=\operatorname{sign}\left(\frac{\varphi_{t}\left(j, t_{j}\right) t_{j}}{\varphi\left(j, t_{j}\right)}-\frac{\varphi_{t}\left(i, t_{i}\right) t_{i}}{\varphi\left(i, t_{i}\right)}\right)
$$

proving the first claim. With iso-elastic costs we obtain $\varphi\left(i, p_{i} T\right)=\gamma c_{i} p_{i}{ }^{\gamma-1} T^{\gamma-1}$. By (57), this

\footnotetext{
${ }^{60}$ It is straightforward to verify by the same arguments we use in Theorem B.3 that this characterization of Nash equilibrium is equivalent to the standard one.

${ }^{61} \mathrm{~A}$ formal advantage of the model in the main text is that it yields a more tractable structure. The respective FOC is $V=\varphi\left(i, p_{i} T\right) T$. For given $p_{i}, T$ marginal costs are thus higher if the own effect on the aggregate are taken into account. This is intuitive, because an increase in $t(i)$ also increases $T$ which, ceteris paribus, decreases $t_{i} / T$.
} 
implies that

$$
\frac{c_{j}}{c_{i}}=\left(\frac{p_{i}}{p_{j}}\right)^{\gamma-1}\left(\frac{1-p_{j}}{1-p_{i}}\right)^{\gamma+1}
$$

from which $R_{i j}=0$ follows. A similar argument together with the fact that $T^{\prime}(V)>0$ shows that $R_{i j}>0$ if $\Phi$ is iso-elastic with exponents $\gamma_{i}$ that increase over agent types, which shows the OR of $p(\cdot)$.

Related Literature Our approach contributes to the literature on aggregative games by adding a set of tools aimed at studying the inequality effects induced by exogenous variations. With respect to comparative-statics, most of the existing literature has focused on the effects on aggregate quantities or the individual strategies (Corchon, 1994; Acemoglu and Jensen, 2013). For example, Acemoglu and Jensen (2013) are interested under what conditions the aggregate, extremal quantities or the individual actions are monotonic in the parameters of the game. By contrast, our approach allows us to study the distributional patterns induced by varying common parameters. Distributional effects may arise even if the individual strategies are monotonic. As the simple contest example shows, none of our tools require a monotonic structure of the game. Moreover, the reformulation of the game as a competition for market shares endows us with an additional tool of deriving the comparative-statics of the aggregate $T$ (see Section 3.3), which may be simpler to evaluate than other methods in certain applications ${ }^{62}$

\section{B.6 Inequality Effects of Market Power?}

In this section, we revisit the case of CES-demand but, contrary to Section 4.1, we assume a perfectly competitive behavior of firms. This simple variation allows us to determine to what extent the observed inequality patterns depend on market power as embodied by monopolistic competition, at least if demand follows the CES-pattern.

Consider a unit mass of consumers endowed with CES utility (23), such that the total demand for firm $i$ is given by (24) where, only for simplicity, we set $r_{i}=1 \forall i \in I$. Like in Section 4.1 . $P_{i}$ and $q_{i}$ denote the price and quantity, respectively, of firm $i \in[0,1]$, and we let $p_{i} \equiv \frac{P_{i} q_{i}}{\int_{I} P_{s} q_{s}}$ be the share of total consumption expenditures earned by firm $i$, and $T \equiv \int_{I} P_{s}^{1-\eta} d s$.

The only difference to monopolistic competition is that each firm $i$ now takes the price $P_{i}$ as given. Assuming the same cost structure as in Section 4.1 yields

$$
\Pi_{i}=P_{i} q_{i}-c_{i} q_{i}^{\gamma_{i}}=p_{i} I-c_{i} I^{\gamma_{i}} p_{i}^{\gamma_{i}} P_{i}^{-\gamma_{i}},
$$

\footnotetext{
${ }^{62}$ The procedure outlined there also applies to the case of Nash equilibria.
} 
as the relevant payoff function. The only differences of (58) to its monopolistic counterpart 26) are that i) the preference parameter $\eta$ does not enter $(58)$ and ii) the price $P_{i}$ enters $(58)$ while $T$ does not. These differences are intuitive as perfectly competitive firms do not account for consumer preferences, and they can influence their market share $p_{i}$ only via their choices of $q_{i}$. The latter further explains why a higher price $P_{i}$, ceteris paribus, has the effect of lowering the costs of attaining a certain market share $p_{i}$ : A higher given price means that a given market share can be maintained by producing less.

A final difference to monopolistic competition is that we shall assume $\gamma_{i}>1$, meaning that all firms are subject to decreasing returns to scale. This is needed to assure positive equilibrium payoffs in case of perfect competition, while market power assured positive payoffs in monopolistic competition also with linear costs.

A competitive (Walrasian) equilibrium is $(p(\cdot), T)$ such that $p_{i}$ maximizes (58), $\int_{I} p_{i} d i=1$ and demand (24) equals supply for each $i \in I$. The definition of $p_{i}$ and equilibrium demand (24) imply that $P_{i}=\left(p_{i} T\right)^{\frac{1}{1-\eta}}$. Using this in the first-order condition $\Pi^{\prime}\left(p_{i}\right)=0$ yields the equilibrium equation

$$
g(i) \equiv \frac{I^{\left(1-\gamma_{i}\right)(\eta-1)}}{\gamma_{i}^{\eta-1}}=c_{i}^{\eta-1} p_{i}^{\left(\gamma_{i}-1\right)(\eta-1)+\gamma_{i}} T^{\gamma_{i}} \equiv \varphi(i) .
$$

Comparing (59) with (27) shows that both equilibrium equations have the same structure, despite the different type of competition 63 Thus, perfect competition yields exactly the same inequality prediction as the monopolistic competition model:

Proposition B.8 All results in Propositions 5 - 7 regarding market and payoff shares as well as relative prices, relative profits and relative quantities also apply to perfect competition.

Proof: The claims regarding the market shares $p(\cdot)$, (relative) quantities and (relative) prices follow from the fact that both models share a structurally identical equilibrium function ( 27$)$ and $(59)$ ). Therefore, repeating the steps in Proposition 5-7 yield the same predictions. The equilibrium payoff in perfect competition is $\Pi_{i}=p_{i} I \frac{\gamma_{i}-1}{\gamma_{i}}$, and all claims about relative payoffs and payoff shares $s(\cdot)$ can be established proceeding exactly as in the proofs of Propositions 5 $-17$.

An additional question we now can ask is whether the market power due to monopolistic competition per se has an inequality effect for a given and fixed set of parameters. The following

\footnotetext{
${ }^{63}$ In fact, the only difference is that $g(i)$ in monopolistic competition, ceteris paribus, is strictly smaller than $g(i)$ in perfect competition. This difference originates from the fact that the firms in monopolistic competition account for the price effect when optimizing their payoffs.
} 
proposition shows that market power increases the level of all equilibrium payoffs, but in a way that that does not affect the dispersion of market shares, independent of whether firms differ in their returns to scale or not. However, market power leads to a more extreme dispersion of the payoff shares, in the sense of a monotonic OR, provided that firms differ in their economies of scale.

Proposition B.9 (Market Power Effects) If all firms have identical returns to scale $\left(\gamma_{i}=\right.$ $\gamma>1 \forall i \in I)$, then the equilibrium market and payoff share functions $p(\cdot) s(\cdot)$ are identical in monopolistic and perfect competition, while each firm earns a strictly higher payoff in monopolistic competition. If firms differ in their returns to scale, then the equilibrium market share function $p(\cdot)$ again is the same for both types of competition, while the equilibrium payoff share in monopolistic competition is a monotonic OR of the one in perfect competition.

Proof: First, suppose that all firms have identical returns to scale, but otherwise are ordered according to $c_{i}$. Let $p^{M}(\cdot)$ and $p^{C}(\cdot)$ denote the equilibrium market share function for monopolistic and perfect competition, respectively. It follows directly from 59 that $\frac{p_{i}^{C}}{p_{j}^{C}}$ also verifies 41. Thus, $\frac{p_{i}^{C}}{p_{j}^{C}}=\frac{p_{i}^{M}}{p_{j}^{M}}$, and $p^{C}(\cdot)=p^{M}(\cdot)$ follows from Lemma 3 . Further, equilibrium payoffs in the perfect competition model are $\Pi_{i}=p_{i} I \frac{\gamma-1}{\gamma}$, while those in monopolistic competition are $\Pi_{i}=p_{i} I\left(\frac{\gamma \eta-\eta+1}{\gamma \eta}\right)$ (see proof of Proposition 5). This shows that $\frac{s_{i}^{C}}{s_{j}^{C}}=\frac{s_{i}^{M}}{s_{j}^{M}}$ and thus $s^{C}(\cdot)=s^{M}(\cdot)$ follow, as well as $\Pi_{i}^{M}>\Pi_{i}^{C} \forall i$.

Now consider the case where the firms are ordered according to $\gamma_{i}$ while $c_{i}=c \forall i$. Proceeding exactly as in the proof of Proposition 6 shows that (45) also holds with perfect competition, implying that relative quantities and thus also relative market shares $\frac{p_{i}}{p_{j}}$ must take on exactly the same values in both models for any $i, j \in I$. As before, this assures that the market share function $p(\cdot)$ must coincide in both models. Turning to payoff shares $s(\cdot)$, it is straightforward to verify that

$$
\frac{s_{i}^{M}}{s_{j}^{M}}=\frac{p_{i}^{M}}{p_{j}^{M}} \frac{\gamma_{j}}{\gamma_{i}} \frac{\left(\gamma_{i}-1\right) \eta+1}{\left(\gamma_{j}-1\right) \eta+1}, \quad \frac{s_{i}^{C}}{s_{j}^{C}}=\frac{p_{i}^{C}}{p_{j}^{C}} \frac{\gamma_{j}}{\gamma_{i}} \frac{\gamma_{i}-1}{\gamma_{j}-1}
$$

Because $\frac{p_{i}^{M}}{p_{j}^{M}}=\frac{p_{i}^{C}}{p_{j}^{C}}$ and $\gamma_{j}>\gamma_{i}$ whenever $j \triangleright i$, we obtain that $\frac{s_{i}^{M}}{s_{j}^{M}}>\frac{s_{i}^{C}}{s_{j}^{C}} \forall j \triangleright i$, which shows that $s^{M}(\cdot)$ must be a monotonic OR of $s^{C}(\cdot)$ by Lemma 3.

\section{B.7 On the labor-leisure model}

We now show that the result on $d \alpha$ generalizes beyond the case where costs are commonelasticity functions, which complicates the analysis. Suppose that $\Phi(i, q(i))$ is not restricted to be a power function, and consider $d \alpha>0$. Then the results on $p^{d}\left(i_{c}\right), q\left(i_{c}\right) / q\left(j_{c}\right), f\left(i_{c}\right) / f\left(j_{c}\right)$, 
$w, T$ and aggregate labor supply in Proposition 12 remain valid provided that $T \leq w \sqrt{64}$ To see this, write firm FOC as $p(i) T=\varphi^{-1}(i, 1 / w)$. Integration yields

$$
T=\int \varphi^{-1}(i, 1 / w) d i \equiv H(w)
$$

with $H^{\prime}(w)<0$. Setting $T^{d}=T$ implicitly defines $w(\alpha)$ by

$$
H(w(\alpha))=\alpha(w(\alpha)+\Pi(w(\alpha)))
$$

By the Envelope Theorem $-\partial_{w} \Pi(i)=\Phi(i, q(i))<q(i) \varphi(i, q(i))=q(i) / w$, where the inequality follows from the strict concavity of $\Phi(i, \cdot)$ and $\Phi(i, 0)=0$, and the equality from firm FOC. Integration yields $-\Pi<T / w$. By 60 we have

$$
w^{\prime}(\alpha)=\frac{w+\Pi}{H^{\prime}(w)-\alpha\left(1+\Pi^{\prime}(w)\right)} .
$$

The denominator is negative if $T \leq w$, because then $1+\Pi^{\prime}(w) \geq 0$, which assures that $w^{\prime}(\alpha)<0$. Then $T^{\prime}(\alpha)>0$, and the remaining claims follow from the proof of Proposition 12 .

\section{B.8 Competition with Logit-Demand}

In this section we analyze the firm-side inequality effects implied by the Logit model of discrete choice with products of possibly heterogeneous quality. The main findings are summarized as follows. With Logit competition, the weakest firms have the strongest incentive to increase the noisiness in the choice procedure, e.g., by using obfuscation tactics. The reason is that increasing noise in the consumer-side product evaluation allows weaker firms to "catch up" because market shares converge to each other once the deterministic part of utility becomes less decisive. Further, we study how an import tax on domestic and foreign firms affects their respective market shares in the domestic market. Such a tax implies the strongest domestic firms to benefit most. Moreover, consumer education about the products is predominantly driven by foreign firms, provided that the importing firms dominate domestic firms in terms of their quality-cost relation.

The basic Logit model In this model, expected demand from a given consumer is a choice probability system (Anderson et al., 1992). Specifically, we consider the Logit demand-system of a single consumer (or a unit mass of identical consumers) with linear utility and Logit noise parameter $\lambda>0$, single-product suppliers, and no outside option. We later show that the

\footnotetext{
${ }^{64}$ If $\Phi(i, q(i))=c(i) q(i)^{\gamma}$ it can be shown that indeed $T \leq w$ must hold in equilibrium.
} 
distributional comparative-statics of the Logit model with outside option are similar. Moreover, if there is a measure $L>0$ of iid consumers, then $p(i) L$ would be the fraction of consumers served by firm $i$. With respect to the distributional outcome, setting $L=1$ is therefore wlog. Firms $i \in[0,1]$ offer possibly different qualities, parametrized by $a(i) \geq 0$. A firm's market share in this model is its selling chance

$$
p(i)=\frac{e^{\lambda\left(a(i)-P_{i}\right)}}{\int e^{\lambda\left(a(s)-P_{s}\right)} d s},
$$

where $P_{i}$ is the price of product $i$. The market share $p(i)$ depends negatively on $P_{i}$ and positively on $a(i)$. Assuming risk neutral firms, a constant production cost $c(i)$ of the good, and setting $T \equiv \int e^{\lambda\left(a(s)-P_{s}\right)}$, the (expected) profit of firm $i$ is

$$
\Pi_{i}=P_{i} p(i)-c(i) p(i)=\left(a(i)-\frac{\operatorname{Ln}(p(i) T)}{\lambda}\right) p(i)-c(i) p(i) .
$$

Let $a(\cdot)$ be decreasing and $c(\cdot)$ increasing, such that $p(i)$ is either of Class I or II. Each firm chooses its market share (its price subject to demand (61)) to maximize profits. The FOC of this problem are65

$$
a(i)-\frac{\operatorname{Ln}(p(i) T)}{\lambda}-\frac{1}{\lambda}=c(i)
$$

from which

$$
p(i)=\frac{e^{\lambda(a(i)-c(i))}}{e T}=\frac{e^{\lambda z(i)}}{e T}, \quad z(i) \equiv a(i)-c(i) .
$$

Hence

$$
\frac{p(i)}{p(j)}=e^{\lambda(z(i)-z(j))}
$$

and

$$
\frac{\partial}{\partial \lambda} \frac{p(i)}{p(j)}=e^{\lambda(z(i)-z(j))}(z(i)-z(j)),
$$

where the assumptions made on $a(i), c(i)$ assure that $z(i)-z(j)>0$ for any $j \triangleright i$. This shows that the monotonic rotation property holds with the Logit, and an increase of $\lambda$ yields an OR of $p(\cdot)$. The parameter $\lambda>0$ controls the noise in the logit. In the degenerate case where $\lambda=0$, the choice process is purely random in such that neither price nor quality have any influence on choice probabilities, and uniform market shares $(p(i)=1)$ result in any equilibrium. An increase in $\lambda$ means that the price, i.e., the deterministic part of utility, becomes more decisive, which always is associated with an increasing inequality of the market shares. This finding is similar to the CES-case, where an increase in the substitution elasticity (also making prices

\footnotetext{
${ }^{65}$ Exponentiating $[63$, one can easily see that this model verifies Assumption 1 , and hence a unique equilibrium exists by Theorem B.2
} 
"more decisive") leads to an OR. It follows from (63) that $P_{i}=\frac{1}{\lambda}+c(i)$, showing that prices always decrease in $\lambda$, and

$$
\operatorname{sign}\left(\frac{\partial}{\partial \lambda} \frac{P_{i}}{P_{j}}\right)=\operatorname{sign}(c(i)-c(j)) \leq 0
$$

Because $\Pi(i)=\frac{p(i)}{\lambda}$, relative payoffs increase in $\lambda$ while industry profits $\int \Pi(s) d s=\frac{1}{\lambda}$ decrease; hence $d \lambda>0$ always generates losers $(d \Pi(i)<0$ must hold for a positive-measure set of firms). The interesting converse is that the weakest firms (in terms of low quality or high costs) have the strongest incentive to increase the "noisiness" in the choice procedure $(d \lambda<0)$, e.g., by resorting to obfuscation tactics (see, e.g., Ellison and Wolitzky, 2012).

Finally, we prove that $d \lambda>0$ can generate winners, provided that there is sufficient firm heterogeneity. From

$$
\Pi(i)=\frac{1}{\lambda \int e^{\lambda(x(s))} d s}, \quad x(s) \equiv z(s)-z(i) \leq 0,
$$

we obtain that

$$
\operatorname{sign}\left(\frac{\partial \Pi(i)}{\partial \lambda}\right)=\operatorname{sign}\left(-\int e^{\lambda(x(s))} d s-\lambda \int x(s) e^{\lambda(x(s))} d s\right)
$$

With symmetric firms we must have $\frac{\partial \Pi(i)}{\partial \lambda}<0$ as then $x(s)=0$. To see that profits of the best firms can increase in $\lambda$, consider the two-types case with $x(0)=0$ and $x(1)=z(1)-z(0)=C<$ 0 . If $\alpha \in(0,1)$ is the fraction of strong types, then $\partial_{\lambda} \Pi(0)>0$ iff $\alpha<-(1-\alpha)(1+\lambda C) e^{\lambda C}$. We can always find $\alpha \in(0,1)$ small enough, such that this inequality is satisfied, provided that $\lambda C<-1$ (enough heterogeneity). Note that because $\partial_{\lambda} \int \Pi(i) d i<0$, the losses of the poor must always outweigh the gains of the rich.

Import competition and taxation In this variation, we study the distributional consequences that an import tax has in the domestic market for home firms and importers using the Logit framework 66 Suppose that the domestic market for the products is composed of home ("H") and foreign ("F") firms, that export their products into home at an import tax of $\tau$. Let $i_{H} \in[0,1]$ and $i_{F} \in[0,1]$ index a home and foreign firm, respectively. Then the market share of firm $i_{\chi}$ in the home market is

$$
p\left(i_{\chi}\right)=\frac{e^{\lambda\left(a\left(i_{\chi}\right)-P_{s}^{\chi}\right)}}{\int e^{\lambda\left(a\left(s_{H}\right)-P_{s}^{H}\right)} d s_{H}+\int e^{\lambda\left(a\left(s_{F}\right)-P_{s}^{F}\right)} d s_{F}}, \quad \chi \in\{H, F\},
$$

\footnotetext{
${ }^{66} \mathrm{~A}$ similar analysis applies with CES-demand.
} 
where $P_{s}^{H}\left(P_{s}^{F}\right)$ is the price of firm $s_{H}\left(s_{F}\right)$, and $\int p(i) d i=\int p\left(i_{H}\right) d i_{H}+\int p\left(i_{F}\right) d i_{F}=1$. With $T \equiv \int e^{\lambda\left(a\left(s_{H}\right)-P_{s}^{H}\right)} d s_{H}+\int e^{\lambda\left(a\left(s_{F}\right)-P_{s}^{F}\right)} d s_{F}$ we obtain

$$
\begin{aligned}
& \Pi\left(i_{H}\right)=\left(a\left(i_{H}\right)-c\left(i_{H}\right)-\frac{\operatorname{Ln}\left(p\left(i_{H}\right) T\right)}{\lambda}\right) p\left(i_{H}\right), \\
& \Pi\left(i_{F}\right)=\left(a\left(i_{F}\right)-\left(\tau+c\left(i_{F}\right)\right)-\frac{\operatorname{Ln}\left(p\left(i_{F}\right) T\right)}{\lambda}\right) p\left(i_{F}\right),
\end{aligned}
$$

which have the same formal structure as $(62)$. It follows that 63 - 65 logically apply also to this version of the model, where we replace $c(i)$ by $c\left(i_{F}\right)+\tau$ if $i_{F}>1 / 267$ Suppose that heterogeneity is such that $p(i)$ is Class I, and consider a small tax increase $d \tau>0$ which does not change the ranking expressed by $p(i)$. By 65 we see that such a change has no effects on the relative market shares $\frac{p\left(i_{H}\right)}{p\left(j_{H}\right)}$ of domestic firms nor on the relative domestic market shares of foreign firms $\frac{p\left(i_{F}\right)}{p\left(j_{F}\right)}$, but the relative market shares $\frac{p\left(i_{H}\right)}{p\left(i_{F}\right)}$ of domestic firms to foreign firms increases in $\tau$ (independent of whether $i_{H} \triangleright i_{F}$ or $i_{F} \triangleright i_{H}$ ). Since by (64) we must have $T^{\prime}(\tau)<0$, it follows again from (64) that $p\left(i_{H}\right)$ increases for any domestic firm and $p\left(i_{F}\right)$ decreases for any foreign firm. It follows that all domestic firms benefit from the import $\operatorname{tax}\left(d \Pi\left(i_{H}\right)>0\right)$, but in a way that leaves relative market shares and relative profits of domestic firms unaltered. This implies that the strongest domestic firms benefit most from the tax in an absolute sense, and essentially follows because the tax allows domestic firms to expand their quantities. From 27) one can infer that all domestic prices $P_{i}^{H}$ remain constant, while foreign prices $P_{i}^{F}$ increase isometrically with $\tau$.

Finally, we note that $d \lambda \neq 0$ has similar effects as before. An additional interesting insight here is, that if importers (F-firms) are comparably strong (meaning that $z\left(i_{F}\right)-\tau \gg z\left(i_{H}\right)$ ), such that importing firms gain from $d \lambda>0$, then importers have an incentive to reduce the noisiness of consumer choice, e.g. by educating consumers. The opposite holds if importers are comparably weak; we would then expect importers to blur or complicate consumer perception.

Logit-demand with an outside option In the Logit model of section B.8 we have assumed that the consumer always purchases one alternative, but what if there is an outside option? A simple way of incorporating an outside option into the Logit (see, e.g., Anderson et al., 1992) is to write expected demand of firm $i$ as

$$
q_{i}=\frac{e^{-\lambda P_{i}}}{\int e^{-\lambda P_{s}}+x}, \quad x=e^{\lambda V_{0}}
$$

\footnotetext{
${ }^{67}$ Note that the following analysis could also be reformulated as finding the effects of an idiosyncratic change of costs, in the spirit of the example in Section 4.3 . for a proper subset of all firms (no H-F-distinction), where $d \tau$ quantifies a non-common cost innovation or regulation.
} 
If $x=0$ this corresponds to the previous model, with a uniform quality level but heterogeneous $\operatorname{costs} c(i)$. We have $\int q_{i} d i<1$ iff $x>0$, because with $x>0$ there is a non-zero probability that the consumer does not purchase at all. Define $p(i)$ as the market share of $i$ relative to total supply, $p(i) \equiv \frac{q_{i}}{\int q_{i}} d i$. Then $q_{i}=p(i) \frac{T}{T+x}$, where $T=\int e^{-\lambda P_{s}}$, and profits are

$$
\Pi_{i}=P_{i} q_{i}-c(i) q_{i}=\frac{T}{T+x}\left(\left(-\frac{\operatorname{Ln}(p(i) T)}{\lambda}\right) p(i)-c(i) p(i)\right)
$$

which is essentially (62) scaled by a positive number. Hence (63) - 65) remain valid for $x>0$ (with the reinterpretation of $p(i)$ ), and the main distributional effects of $\lambda$ are preserved. In particular, an increase in $\lambda$ leads to an OR of $p(\cdot)$ and increases relative profits as well as relative prices $\frac{P_{i}}{P_{j}}$ (because $\left.c(i)<c(j), j \triangleright i\right)$. Moreover, a change in the value of the outside option $(d x \neq 0)$ has no equilibrium effects on market shares nor on relative profits nor on relative (expected) quantities supplied. By (63) prices $P_{i}$ are invariant to $x$, and by $64 T^{\prime}(x)=0$, showing that the only effect of (a change in) the outside option is to change individual and aggregate supply, and to scale all equilibrium profits by a factor, while preserving market shares and market prices 68

\footnotetext{
${ }^{68}$ Note however that quantity differences, $q_{i}-q_{j}>0$, and profit differences $\Pi(i)-\Pi(j)>0$ decrease in $x$.
} 